\title{
Invariantes Cohomológicos e Decomposição de Grupos
}

\section{Ermínia de Lourdes Campello Fanti}

\author{
orientador \\ Prof. Dr. Janey Antonio Daccach
}

Tese de doutorado apresentada ao Instituto de Ciências Matemáticas de São Carlos - USP, como parte dos requisitos para obtençâo do título de "Doutor em Ciências - Área Matemática - Geometria e Topologia".

\author{
USP - São Carlos \\ Dezembro - 1992
}




\section{Agradecimentos}

Ao Professor Janey Antonio Daccach, pela dedicada e atenciosa orientação.

Ao meu esposo, pelo amor, compreensão e apoio nos momentos difíceis.

À Gorete, pela amizade e participação nesta caminhada.

Aos Professores do ICMSC - USP, pela acolhida, formação e estímulo.

Ao Departamento de Matemática da UNESP - Sào José do Rio Preto que me proporcionou condições adequadas para à dedicação ao Programa de Doutorado.

A Capes, pelo auxilio financeiro.

Aos meus Professores do curso de graduação (UNESP - SJRP) e Mestrado (IMPA) pela formação e incentivo.

$\dot{A}$ todos que de alguma forma contribuiram para a realizasão deste trabalho, em particular aos meus pais por iniciar-me nos estudos, à vó Bel pelo carinho dedicado à Aline e Gabriela durante minha ausência e ao amigo Toninho pelo excelente trabalho de digitação.

À Deus, por tudo. 
Dedico ao

Dinho

Aline $\mathrm{e}$

Gabriela 


\section{Abstract}

In this work we define a cohomological invariant $E(G, \mathcal{S}, M)$ where $G$ is a group, $\mathcal{S}=\left\{S_{i}\right\}_{i \in J}$ is a family of infinite index subgroups of $G$ and $M$ a $\mathbf{Z}_{2} G$-module. The case where $\mathcal{S}=\{S\}$ is investigated. We verify that $E(G,\{S\}, M)$ has a interpretation in terms of derivations and principal derivations, and so in certain cases computation is available. Also we give a topological interpretation for $E(G,\{S\}, M)$ in terms of relative cohomology of complexes $(X, Y)$ if $(X, Y)$ is an Eilenberg-MacLane pair realizing $(G, S)$. This invariant is closely related to the classical end $\epsilon(G)$ for a group $G$, and the ends $e(G, S), \tilde{e}(G, S)$ for a group pair. We denote $E\left(G,\{S\}, \mathbb{Z}_{2}(G / S)\right)$ and $E\left(G,\{S\}, \mathbf{Z}_{2} G \otimes \mathbf{Z}_{2} S \cdot P S\right)$ by $E(G, S)$ and $\tilde{E}(G, S)$ respectively. We have that $E(G,\{1\})=\hat{E}(G,\{1\})=e(G)$ and in some cases $E(G, S)=\epsilon(G, S)$ and $\hat{E}(G, S)=$ $\tilde{\varepsilon}(G, S)$. However we give examples where they are distinct. Some results are obtained in the case where $G$ and $S$ have certain property of duality. We relate $\tilde{E}(G, S)$ with decomposition of groups like HNN-extensions and free amalgamated product. 


\section{Resumo}

Neste trabalho definimos um invariante cohomológico $E(G, S, M)$ onde $G$ é um grupo, $\mathcal{S}=\left\{S_{i}\right\}_{i \in J}$ é uma família de subgrupos de $G$ de índice infinito e $M$ é um $\mathbf{Z}_{2} G$-módulo. O caso onde $S=\{S\}$ é investigado. Verificamos que $E(G,\{S\}, M)$ têm uma interpretação em termos de derivaçōes e derivaçōes principais, e deste modo em certos casos a computação deste invariante é possível. Também apresentamos uma interpretação topológica para $E(G, S, M)$ em termos de cohomologia relativa de complexos $(X, Y)$ se $(X, Y)$ é um par Eilenberg-MacLane realizando $(G, S)$. Este invariante está intimamente relacionado com o end clássico $e(G)$ para um grupo $G$, e os ends $e(G, S)$ e $\hat{e}(G, S)$ para um par grupo $(G, S)$. Denotamos $E\left(G,\{S\}, \mathbb{Z}_{2}(G / S)\right)$ e $E\left(G,\{S\}, \mathbb{Z}_{2} \otimes \mathbf{Z}_{2} S S\right)$ por $E(G, S)$ e $\tilde{E}(G, S)$ respectivamente. Temos que $E(G,\{1\})=\tilde{E}(G,\{1\})=e(G)$ e em alguns casos $E(G, S)=e(G, S)$ e $\hat{E}(G, S)=\hat{e}(G, S)$. Entretanto damos exemplos onde eles sâo distintos. Alguns resultados são obtidos no caso onde $G$ e $S$ têm certas propriedades de dualidade. Relacionamos $\hat{E}(G, S)$ com decomposiçoes de grupos tais como HNN-extensōes e produto livre amalgamado. 


\section{INDICE}

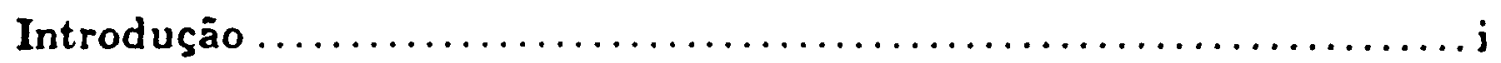

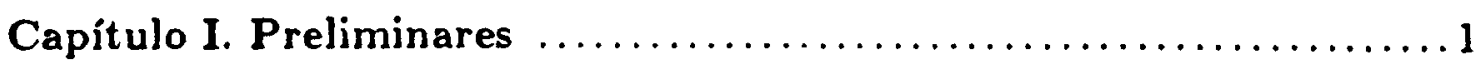

1. Homologia e Cohomologia de Grupos .......................

2. $H^{*}$ e $H_{*}$ como funtores do módulo coeficiente e funtores de duas

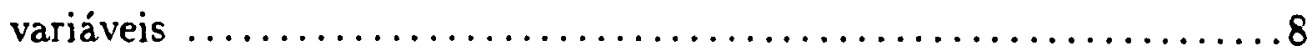

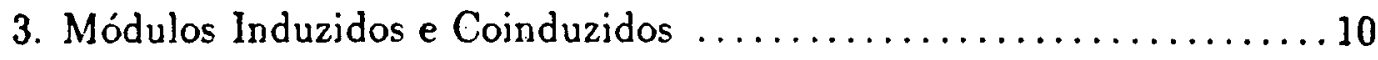

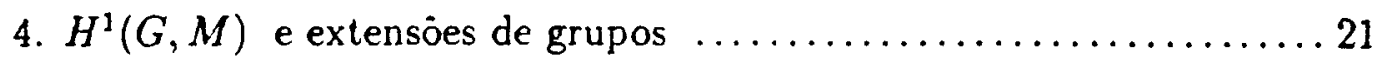

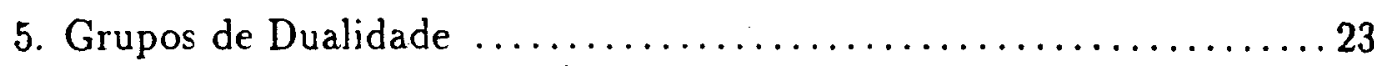

Capítulo II. Un Invariante cohomológico para par grupos $\ldots \ldots \ldots 33$

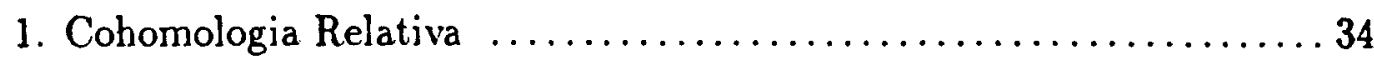

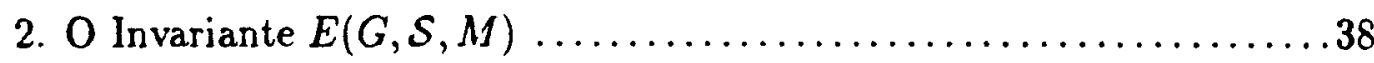

3. $E(G, S)$ e Dualidade ................................. 46

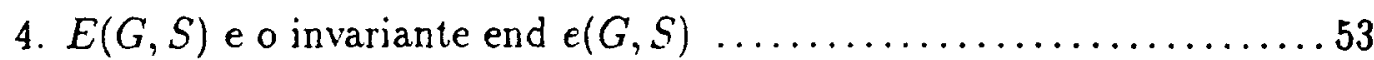

5. Uma interpretação para $E(G, S, M)$ em termos de cohomologia relativa

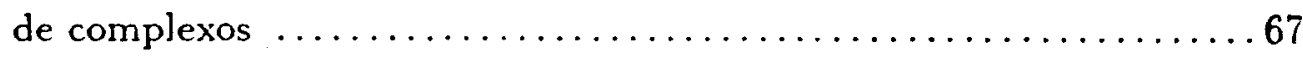

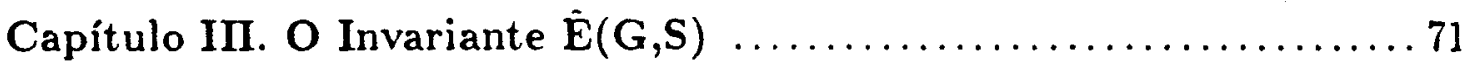

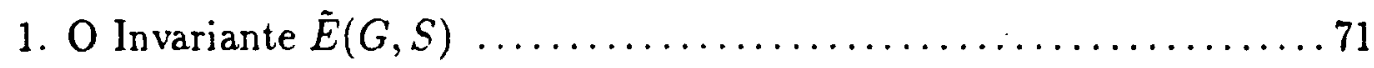

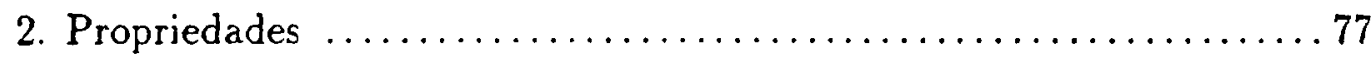

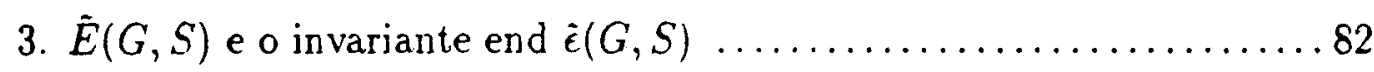

Capítulo IV. $\hat{\mathbf{E}}(\mathrm{G}, \mathrm{S})$ e decomposição de grupos $\ldots \ldots \ldots \ldots \ldots \ldots . \ldots 90$

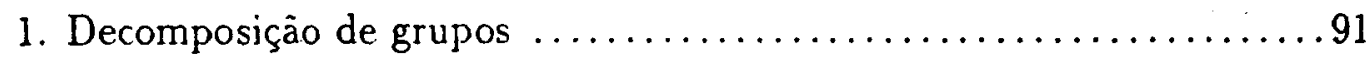

2. $\tilde{E}(G, S)$ e decomposiçâo de grupos segundo Kropholler e Roller ....993

Bibliografia .......................................... 105 


\section{INTRODUÇÃO}

Sabemos da existência de invariantes denominados ends (mais precisamente número de ends) para $X^{\prime}$, um espaço topológico: $\epsilon\left(X^{\prime}\right)$; para $G$ um grupo: $e(G)$ e para pares $(G, S): e(G, S)$ e $\tilde{e}(G, S)$.

A teoria de ends de espaços topológicos foi introduzida por Freudenthal em [13] (1931).

A teoria de ends de grupos foj introduzida por Hopf [16] (1943) para grupos finitamente gerados e foi modelada na teoria de ends de espaços.

Posteriormente Specker [30] (1949) deu uma definiçâo puramente algébrica para $e(G)$, para todo grupo $G$ (nào necessariamente finitamente gerado). Se $G$ é infinito então

$$
\epsilon(G)=1+\operatorname{dim} \mathbf{Z}_{2} H^{1}\left(G ; \mathbb{Z}_{2} G\right)=1+\operatorname{dim} \mathbf{Z}_{2} \frac{\operatorname{Der}\left(G, \mathbb{Z}_{2} G\right)}{P\left(G, \mathbb{Z}_{2} G\right)}
$$

$O$ end $\epsilon(G, S)$ foi introduzido por Houghton [17] (1974) para grupos topológicos, e Scott. [26] (1977) explorou este invariante no caso discreto. Para $\epsilon(G, S)$ não existe uma fórmula cohomológica do tipo (1) quando $[G: S]=\infty$.

Quanto ao invariante end $\hat{\epsilon}(G, S)$, foi definido por Kropholler e Roller em [2] (1989), e se $[G: S]=\infty$ entào

$$
\tilde{\epsilon}(G, S)=1+\operatorname{dim} \mathbf{Z}_{2} H^{1}\left(G ; \mathcal{F}_{S} G\right)=1+\operatorname{dim} \mathbf{Z}_{2} \frac{\operatorname{Dcr}\left(G, \mathcal{F}_{S} G\right)}{P\left(G, \mathcal{F}_{S} G\right)}
$$

onde $\mathcal{F}_{S} G=\{A \subseteq G \mid A \subseteq F . S$ para algum subconjunto finito $F$ de $G\}$.

Com base na teoria de cohomologia relativa de grupos, nós definimos um invariante "end" generalizado $E(G, \mathcal{S}, M)$ para $G$ um grupo, $\mathcal{S}=\left\{S_{i}, i \in I\right\}$ uma família de subgrupos de $G$ com $\left[G: S_{i}\right]=\infty, \forall i \in I$ e $M$ um $\mathbb{Z}_{2} G$-módulo qualquer.

A definição deste invariante ( e parte do capítulo Il deste trabalho) foi obtida em colaboração com Maria Gorete Carreira Andrade [2], e surgiu da tentativa de se obter 
para $e(G, S)$ uma fórmula do tipo (1) envolvendo cohomologia relativa de grupos.

Neste trabalho estamos interessados no caso em que $\mathcal{S}$ consiste de um único subgrupo $S$, isto é, $S=\{S\}$. O caso genérico $E(G, S, M)$ é explorado por Marja Gorete Carreira Andrade em [1].

Relacionaremos os invariantes $E\left(G,\{S\}, \mathbf{Z}_{2}(G / S)\right)$ e $E\left(G,\{S\}, \mathcal{F}_{S} G\right)$ com $\epsilon(G, S)$ e $\tilde{\epsilon}(G, S)$, respectivamente. Veremos que em certos casos eles coincidem. Porém, nossos invariantes dão uma condição necessária para que $(G, S)$ seja um $D^{n}$-par, o que não ocorre com os demais.

Além disto, $E\left(G,\{S\}, \mathcal{F}_{S} G\right)$ nos dá informações a respeito do subgrupo $S$ quando $\epsilon(G)=\infty$, o que nào acontece com $\tilde{e}(G, S)$ pois, se $c(G)=\infty$ então $\tilde{e}(G, S)=\infty$ para qualquer subgrupo $S$.

Veremos também que sob algumas hipóteses $E\left(G,\{S\}, \mathcal{F}_{S} G\right)$ mede exatamente quando $G$ se decompõe sobre subgrupo comensurável com $S$, o que não é verdade para $\hat{e}(G, S)$.

Este Trabalho está dividido em 4 capitulos.

No capítulo I recordamos alguns resultados de (co)homologia de grupose dualidade. O propósito deste capítulo é de fixar notaçâo e evitar ao leitor frequentes consultas às referências.

No capítulo Il, introduzimos o invariante $E(G, \mathcal{S}, M)$ para $\mathcal{S}=\left\{S_{i}\right\}_{i \in l}$, $\operatorname{com}\left[G: S_{i}\right]=\infty, \forall i \in I$ e $M$ um $\mathbb{Z}_{2} G$-módulo, ou seja,

$$
E(G, \mathcal{S}, M)=1+\operatorname{dim} \mathbf{Z}_{2} \operatorname{Ker} r \epsilon s_{S, M}^{G}
$$

onde $r \in s_{s, M}^{G}: H^{1}(G ; M) \rightarrow \prod_{i \in I} H^{1}\left(S_{i} ; M\right)$ é a aplicaçâo restriçâo (Il.2.1).

No caso em que $S=\{S\}$, nós denotamos $E(G,\{S\}, M)$ simplesmente por $E(G, S, M)$.

Algumas caracterizaçoes deste invariante foram apresentadas.

A primeira é em termos de característica de Euler parcial (II.2.4), isto é, "Se a dimensâo $\left(e m \mathbb{Z}_{2}\right)$ de $H^{0}(G ; M), H^{0}(S ; M)$ є $H^{1}(G, S ; M)$ é finita então $E(G, S, M)=1+\operatorname{dim} \mathbf{Z}_{2} H^{0}(G ; M)-\operatorname{dim} H^{0}(S ; M)+\operatorname{dim} H^{1}(G, S ; M) . "$

(Esta caracterização nos foi sugerida pelo Prof. Alejandro Adem). 
A segunda é em termos de derivaçōes e derivaçōes principais (11.2.5), a saber

$$
E(G, S, M)=1+\operatorname{dim} \mathbf{Z}_{2} \frac{H^{1}(G, S ; M)}{P(G, S, M)}=1+\operatorname{dim} \mathbf{Z}_{2} \frac{\operatorname{Der}(G, S, M)}{P(G, S, M)} .
$$

A terceira é em termos de cohomologia relativa de complexos (II.5.2), ou seja, se $(X, Y)$ é um par Eilenberg-MacLane realizando $(G, S)$ entâo

$$
E(G, S, M)=1+\operatorname{dim} \frac{H^{1}(X, Y ; M)}{M^{S} / M^{G}}
$$

onde a cohomologia é tomada com coeficientes locais.

Neste capítulo nos dedicamos de modo especial ao invariante $E\left(G,\{S\}, \mathbf{Z}_{2}(G / S)\right)$ que denotamos por $E(G, S)$.

Provamos que: Dado $(G, S)$ com $[G: S]=\infty$, se $(G, S)$ é um $D^{n}$-par entâo $E(G, S)=1$ ou equivalentemente, se $E(G, S) \neq 1$ entâo $(G, S)$ não é um $D^{n}$. par (II.3.6). (Este resultado é uma generalizaçâo do existente para um grupo, a saber, se $G$ é um $D^{n}$-grupo, $n>1$, entâo $\epsilon(G)=1$ ).

Como aplicação deste resultado concluimos que um determinado par $(G, S)$ não é de dualidade. Isto é obtido computando $E(G, S)$ em termos de derivaçôes e derivaçôes principais (II.3.8).

Também verificamos, por exemplo, que se $G$ é um $P D^{n}$-grupo e $S$ um $P D^{n-1}$-subgrupo entâo $E(G, S) \leq 2$.

Em II.4 relacionamos $E(G, S)$ com os ends $\epsilon(G)$ e $e(G, S)$. Verificamos (em 11.4.24) que

$$
E(G, S) \leq e(G, S)
$$

e estabelecemos uma condiçào necessária e suficiente para se obter a igualdade (II.4.25). Nos casos em que a igualdade é válida (por exemplo quando $S$ é normal em $G$ (Il.4.24 e Il.4.30)) teremos para $\epsilon(G, S)$ uma fórmula do tipo (1) envolvendo cohomologia relativa. ou seja,

$$
e(G, S)=1+\operatorname{dim} \mathbf{Z}_{2} \frac{H^{1}\left(G, S ; \mathbb{Z}_{2}(G / S)\right)}{P\left(G, S, \mathbf{Z}_{2}(G / S)\right)}
$$

Também nestes casos $\epsilon(G, S)$ terá uma interpretação topológica em termos de pares Eilenberg-MacLane (Il.5.3).

No capítulo IIl, dados $(G, S)$ com $[G: S]=\infty$, estudamos o invariante 
$E(G,\{S\}, M)$ para $M=\mathcal{F}_{S} G \simeq \mathbf{Z}_{2} G \otimes \mathbf{Z}_{2} S S$, que denotamos por $\dot{E}(G, S)$.

Para este invariante conseguimos (IIl.1.6) uma definição equivalente que inclui o caso em que $[G: S]<\infty$ (isto não foi possivel para $E(G, S)$ ).

Várias propriedades de $\hat{E}(G, S)$ são apresentadas. Por exemplo, provamos que $E(G, S) \leq \hat{E}(G, S) \quad$ (III.2.1) e concluimos que a igualdade nem sempre ocorre (III.2.3).

Também observamos que não existe relação entre os invariantes $\tilde{E}(G, S)$ e $e(G, S)$ (Ill.2.4).

Verificamos que $\tilde{E}(G, S)$ preserva propriedades de dualidade análogas às obtidas para $E(G, S)$ embora seja um majorante de $E(G, S)$ (III.2.8). Usando estas propriedades concluimos, por exemplo, que $\tilde{E}\left(\mathbf{Z} \oplus(\mathbf{Z} * \mathbb{Z}) \oplus \mathbf{Z}_{n}, \mathbf{Z} * \mathbf{Z}\right)=2$ (III.2.10)(ii). Por último (III.3) comparamos $\tilde{E}(G, S)$ com $\tilde{e}(G, S)$. Temos que $\hat{E}(G, S) \leq \tilde{\epsilon}(G, S)$, mas a igualdade nem sempre ocorre pois, por exemplo, a propriedade: "se $(G, S)$ é um $D^{n}$-par entâo $\hat{E}(G, S)=1$ " não é verdadeira para $\hat{e}(G, S)$ (III.3.6). Uma condição necessária (no caso em que os invariantes sâo finitos) e suficiente para se obter a igualdade é que $\operatorname{res}_{S_{, F_{S} G}^{G}}$ seja a aplicação nula. Apresentamos certos casos em que a igualdade ocorre. Por exemplo, se $S$ é normal e finitamente gerado (III.3.9) ou se $S$ é um $P D^{n}$-grupo e $\left\{g \in G \mid c d\left(S^{g} \cap S\right)=n-1\right\}=\emptyset$ (III.3.11). Como aplicaçâo deste último resultado computamos $\hat{E}(G, S)$ em um exemplo específico (III.3.12).

Finalizando observamos que por (5) $\hat{E}(G, S)$ tem uma interpretaçào topológica em termos de pares Eilenberg-MacLane. Daí. $\tilde{\epsilon}(G, S)$ também terá tal interpretaçâo se a igualdade $\hat{e}(G, S)=\hat{E}(G, S)$ for válida (III.3.16).

No último capítulo analisamos alguns dos resultados de hropholler e Roller sobre decomposiçâo de grupos [21] em termos de nosso invariante $\tilde{E}(G, S)$.

Em todos os resultados de [21] é suposto que $H^{1}\left(G ; \mathcal{F}_{S} G\right) \simeq \mathbb{Z}_{2}$ $(\Longleftrightarrow \tilde{\epsilon}(G, S)=2$ por (2)). Se $G$ e $S$ sâo finitamente gerados, os autores apresentaram uma condição necessária e (sob certas restriçôes) suficiente, para $G$ se decompor sobre um subgrupo comensurável com $S$. A condiçâo é que a obstrução $\operatorname{sing}_{G}(S)$ (introduzida pelos autores), seja nula.

Verificamos que $\tilde{E}(G, S)$ substitui a obstruçâo $\operatorname{sing}_{G}(S)$ (que está definida 
somente quando $\tilde{e}(G, S)=2$ ). Por exemplo, adaptando um dos resultados de [21] em termos de $\tilde{E}(G, S)$ temos: "se $G$ è um $P D^{n}$-grupo e $S$ um $P D^{n-1}$-subgrupo então $G$ se decompóe sobre um subgrupo comensurável com $S$ se e somente se $\hat{E}(G, S)=2$."

Nosso objetivo é obter resultados similares aos de [21], porém substituindo $\operatorname{sing}_{G}(S)$ por $\hat{E}(G, S)$, e sempre que possivel, retirar a hipótese $\hat{e}(G, S)=2$.

Neste sentido verificamos que o seguinte resultado é válido sem tal hipótese. "Se $G$ se decompóe sobre um subgrupo comensurável com $S$ entâo $\tilde{E}(G, S) \geq 2$ " (IV.2.6). Daí concluimos que: "Se $(G, S)$ é um $D^{n}$-par então $G$ não se decompõe sobre um subgrupo comensurável com $S "$ (IV.2.7).

Com isto temos exemplos de pares $(G, S)$ tais que $\hat{e}(G, S) \neq 2$ (e portanto $\operatorname{sing}_{G}(S)$ nào está definida) mas no entanto, usando $\tilde{E}(G, S)$ podemos concluir que $G$ não se decompôe sobre um subgrupo comensurável com $S$ (IV.2.8).

Em outro resultado analisado verificamos que a hipótese $\hat{e}(G, S)=2$ é consequência das outras, portanto nâo é possivel retirá-la. Também verificamos que uma das condiçôes imposta neste resultado pode ser enfraquecida (IV.2.11). 


\section{CAPÍTULO I}

\section{Preliminares}

O objetivo deste capítulo é recordar alguns resultados da teoria de (co)homologia de grupos envolvendo a teoria de dualidade, que serâo úteis no desenvolvimento deste trabalho.

Para as seçôes 1 à 4 nossa referência básica será $[\delta]$. Queremos observar que em [8] a (co)homologia de um grupo $\mathrm{G}$ é definida sobre o anel $\mathbb{Z}$ dos inteiros, com coeficientes em um $\mathbf{Z} G$-módulo $M$, porém pode-se considerar, no lugar de $\mathbf{Z}$, qualquer anel com unidade $R$.

Aqui consideraremos o caso em que $R$ é o anel $\mathbb{Z}$ ou o corpo $\mathbf{Z}_{2}=\{0,1\}$ de dois elementos. Porém nosso interesse (nos capítulos seguintes) é no caso em que $R=\mathbb{Z}_{2}$. Portanto nos restringiremos, sempre que for conveniente, somente a este caso.

Para a secção 5 nossa referência básica será as notas de R. Bieri [4].

A seguir damos uma breve descrição do conteúdo deste capítulo: Primeiro introduzimos o conceito de homologia e cohomologia de grupos, via resoluçôes projetivas de $R$ sobre $R G$, e apresentamos a interpretação topológica de $H_{*}(G ; M)$ e $H^{*}(G ; M)$ para

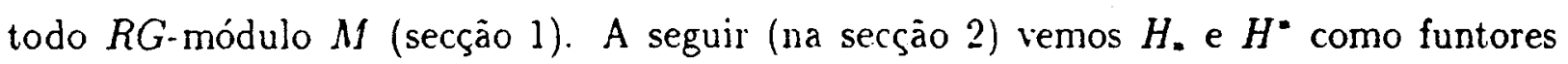
covariantes do módulo coeficiente, e mais geralmente, como funtores de duas variáveis.

$\mathrm{N}$ a seç̧ão 3 estudamos os $\mathbb{Z}_{2} G$-módulos do tipo $\operatorname{Ind} d_{S}^{G} M$ e $\operatorname{Coind} d_{S}^{G} M$, onde $S$ é um subgrupo qualquer de $G$ e $M$ é um $\mathbb{Z}_{2} S$-módulo. Esta secção é fundamental para os próximos capitulos. Nela recordamos propriedades básicas que serão muitas vezes utilizadas em nossos resultados, dentre elas destacamos a Fórmula de Mackey e o Lema de 
Shapiro. Na seç̧ão 4 apresentamos a interpretaçào de $H^{1}(G ; M)$ em termos de extensōes cindidas de grupos.

Finalmente, na seç̧ão 5 , recordamos o conceito de dimensão (co)homológica, grupos de dualidade e suas propriedades mais relevantes.

Notações especiais: Conforme observamos acima, nos restringiremos quase sempre ao caso em que $R=\mathbb{Z}_{2}$. Assim, por conveniência, em todos os capitulos, denotaremos $-\otimes \mathbf{Z}_{2} G-, \operatorname{Hom}_{\mathbf{Z}_{2} G}(-,-),-\otimes_{\mathbf{Z}_{2}}-, \operatorname{Hom}_{\mathbf{Z}_{2}}(-,-)$ e $\operatorname{dim}_{\mathbf{Z}_{2}}$ respectivamente por $-\otimes_{G}-, \operatorname{Hom}_{G}(-,-),-\otimes-, \operatorname{Hom}(-,-)$ e dim.

\section{Homologia e Cohomologia de Grupos}

Para nós $G$ sempre denotará um grupo, $R$ o anel $\mathbf{Z}$ ou o corpo $\mathbf{Z}_{2}$, e $R G$ o anel grupo de $G$ sobre $R$ : Os elementos de $R G$ são somas formais $\sum r_{g} g$ com $r_{g} \in R, g \in G$ onde $r_{s}=0$ para quase todo $g$ (portanto $R G$ é o $R$-módulo livre gerado pelos elementos de $G$ ) e, as operações são dadas por

$$
\begin{aligned}
\left(\sum r_{g} g\right)+\left(\sum s_{g} g\right) & =\sum\left(r_{g}+s_{g}\right) g \\
\left(\sum r_{g} g\right) \cdot\left(\sum s_{g} g\right) & =\sum t_{g} g, \text { onde } t_{g}=\sum_{x y=g} r_{x} s_{y} .
\end{aligned}
$$

A unidade de $R G$ é o elemento $1=1 . \epsilon$ onde $\epsilon$ é o elemento neutro de $G$. $O$ elemento $1 g \in R G$ será denotado por $g$.

Para qualquer grupo $G$ podemos definir o homomorfismo de aneis

$$
\varepsilon: R G \rightarrow R, \text { tal que } \varepsilon(g)=1, \text { para todo } g \in G .
$$

Este homomorfismo é denominado aplicaçâo aumentaçâo.

1.1: Neste trabalho, a menos que se especifique o contrário, trabalharemos na categoria de $R G$-módulos à esquerda.

$\dot{E}$ interessante observar que todo $R G$-módulo à esquerda $N\left(R=\mathbf{Z}\right.$ ou $\left.\mathbf{Z}_{2}\right)$ pode ser considerado como um $R G$-módulo à direita por definir $n .(r g)=\left(r g^{-1}\right) n$, para 
$r \in R, g \in G$ e $n \in N$. De modo análogo, todo $R G$-módulo à direita pode ser considerado como um $R G$-módulo à esquerda.

1.2 Observaçōes: (a) Se $M$ é um $R G$-módulo (à esquerda) então:

(i) $M$ é um $R$-módulo (á esquerda) pois basta definir r.m $:=(r e) m$ onde $e$ é o elemento neutro de $G, \mathrm{e}$

(ii) Existe uma ação (à esquerda) de $G$ em $M$ dada por $g \cdot m=(1 g) m$.

Reciprocamente, se $M$ é um $R$-módulo e existe uma açâo de $G$ em $M$ entâo podemos dar a $M$ uma estrutura de $R G$-módulo, definindo $(r g) \cdot m:=r(g m)$ e estendendo por linearidade.

Assim, para que $M$ seja um $\mathbb{Z}_{2} G$-módulo é necessário, e suficiente, que $M$ seja um $\mathbf{Z}_{2}$-módulo (isto é, um grupo abeliano em que todo elemento tem ordem $2 \mathrm{e}$ portanto $m=-m$ ) e que exista uma ação de $G$ sobre $M$.

Logo, se $M$ é um $\mathbf{Z}_{2} G$-módulo entâo obviamente $M$ é um $\mathbf{Z} G$-módulo.

(b) $\mathrm{O}$ anel $R$ será sempre considerado um $R G$-módulo com a $G$-ação trivial, isto é, $g . r=r$, para todo $r \in R$ e $g \in G$. Assim, $\left(\sum r_{g} g\right) . r=\sum r_{g}(g . r)=\sum r_{g} r$.

No caso em que $R=\mathbb{Z}_{2}$ esta é a única estrutura de $\mathbb{Z}_{2} G$-módulo possivel, pois $\operatorname{Aut}\left(\mathbb{Z}_{2}\right)=\{i d\}$.

(c) A ação diagonal: Sejam $M$ e $N$ RG-módulos (à esquerda). Nós podemos dar a $M \otimes_{R} N$ (respectivamente $H_{o} o_{R}(M, N)$ ) uma estrutura de $R G$-módulo induzida pela $G$-ą̧âo diagonal

$g .(m \otimes n)=g m \otimes g n, \forall g \in G, m \in M$ e $n \in N$

(resp. $(g . f)(m)=g f\left(g^{-1} m\right), \forall g \in G, m \in M$ e $\left.f \in \operatorname{Hom}_{R}(M, N)\right)$.

Uma resolução projetiva de $R$ sobre $R G$ é uma sequência exata de $R G$.

módulos

$$
\cdots \rightarrow F_{n} \stackrel{\partial_{n}}{\rightarrow} F_{n-1} \rightarrow \cdots \rightarrow F_{1} \stackrel{\partial_{1}}{\rightarrow} F_{0} \stackrel{\iota}{\rightarrow} R \rightarrow 0
$$

em que cada $F_{i}$ é $R G$-projetivo.

Tal resolução sempre existe pois podemos tomar $F_{0}=R G, \varepsilon$ a aplicação 
aumentação, e se $F_{1}, \ldots, F_{n}$ são definidos, tomamos para $F_{n+1}$ qualquer módulo livre aplicado sobre $K_{n}:=\operatorname{Ker}\left(F_{n} \rightarrow F_{n-1}\right)$ e para $\partial_{n+1}$ a aplicação correspondente $F_{n+1} \rightarrow K_{n} \hookrightarrow F_{n}$. Note que esta construção nos dá uma resolução livre de $R$.

Por conveniência às vezes denotamos uma resolução (*) simplesmente por $\varepsilon: F \rightarrow R$.

1.3 Definição: Sejam $F \stackrel{\leftarrow}{\rightarrow} R$ uma resolução projetiva de $R$ sobre $R G$ e $M$ um RG-módulo (à esquerda). Consideremos os complexos

$$
\begin{aligned}
& F \otimes_{R G} M: \cdots \rightarrow F_{2} \otimes_{R G} M \rightarrow F_{1} \otimes_{R G} M \rightarrow F_{0} \otimes_{R G} M \rightarrow 0 \quad e \\
& H o m_{R G}(F, M): 0 \rightarrow H_{R m_{R G}}\left(F_{0}, M\right) \rightarrow \operatorname{Hom}_{R G}\left(F_{1}, M\right) \rightarrow \cdots
\end{aligned}
$$

Os grupos de (co)homologia de $G$ com coeficientes em $M$ são definidos por

$$
\begin{aligned}
& H^{j}(G ; M)=H^{j}\left(\operatorname{Hom}_{R G}(F, M)\right) \\
& H_{j}(G ; M)=H_{j}\left(F \otimes_{R G} M\right), \quad j=0,1, \ldots
\end{aligned}
$$

Quando $M=\mathbb{Z}$ com a $G$-ação trivial é usual denotar $H^{*}(G ; M)$ (resp. $H_{*}(G ; M)$ ) por $H^{*}(G)$ (resp. $H_{*}(G)$ ). Neste caso os grupos são denominados grupos de cohomologia de $G$ (resp. grupos de homologia de $G$ ).

1.4 Observações: (a) Estamos levando em conta 1.1 quando usamos a mesma resolução $F$ para definir os grupos de homologia e cohomologia, pois em $F_{n} \otimes_{R G} M$, $F_{n}$ é considerado como $R G$-módulo à direita enquanto que em $\operatorname{Hom}_{R G}\left(F_{n}, M\right), F_{n}$ é visto como $R G$-módulo à esquerda.

(b) Pode-se verificar que a definição acima independe da resoluçào projetiva escolhida (cf. $[8,1.7 .5])$.

(c) Para qualquer grupo $G$ podemos construir uma resolução bastante im. portante no estudo dos grupos de (co)homologia denominada resolução Bar (cf. [8, 1.5]). Ela é dada por:

$$
\cdots \rightarrow F_{n} \stackrel{\theta}{\rightarrow} F_{n-1} \rightarrow \cdots \rightarrow F_{1} \rightarrow F_{0} \stackrel{\bullet}{\rightarrow} R
$$


onde $F_{n}$ é o $R G$-módulo livre gerado pelos simbolos $\left[g_{1}\left|g_{2}\right| \ldots \mid g_{n}\right], g_{i} \in G$. Se $n=0$, existe somente um tal elemento básico, denotado por [ ], assim $F_{0}$ pode ser identificado com $R G$ se identificamos [ ] com 1. O operador bordo é dado por

dado por:

$$
\partial\left[g_{1}|\ldots| g_{n}\right]=\sum_{i=1}^{n}(-1)^{i} d_{i}\left[g_{1}|\ldots| g_{n}\right], \text { onde } d_{i} \text { é o } R G \text {-homomorfismo }
$$

$$
d_{i}\left[g_{1}|\ldots| g_{n}\right]= \begin{cases}g_{1}\left[g_{2}|\ldots| g_{n}\right] & \text { se } i=0 \\ {\left[g_{1}|\ldots| g_{i-1}\left|g_{i} g_{i+1}\right| g_{i+2}|\ldots| g_{n}\right]} & \text { se } 0<i<n \\ {\left[g_{1}|\ldots| g_{n-1}\right]} & \text { se } i=n .\end{cases}
$$

Em dimensōes baixas, esta resolução tem a forma

$$
F_{2} \stackrel{\partial_{2}}{\rightarrow} F_{2} \stackrel{\partial_{1}}{\rightarrow} R G \stackrel{e}{\rightarrow} R \rightarrow 0
$$

onde

$$
\left\{\begin{array}{l}
\partial_{2}[g \mid h]=g[h]-[g h]+[g], \\
\left.\partial_{1}(\mid g]\right)=g[]-[]=g-1, \quad \mathrm{e} \\
\varepsilon(1)=1 .
\end{array}\right.
$$

(Se exigirmos que em $\left[g_{1}\left|g_{2}\right| \ldots \mid g_{n}\right]$ tenhamos $g_{i} \neq 1$, para todo $1 \leq i \leq n$ então nós temos a chamada resoluçâo bar normalizada).

Se $F \rightarrow \mathbb{Z}_{2}$ é a resolução bar de $\mathbb{Z}_{2}$ sobre $\mathbb{Z}_{2} G$, vamos denotar $\operatorname{Hom}_{G}\left(F_{n}, M\right)$ por $C^{n}(G, M)$. Um elemento de $C^{n}(G, M)$ pode ser considerado como uma função $f: G^{n} \rightarrow M$, isto é, uma funçâo de $n$ variáveis de $G$ a $M$ onde identificamos $\left[g_{1}\left|g_{2}\right| \ldots \mid g_{n}\right]$ com $\left(g_{1}, \ldots, g_{n}\right)$. O operador cobordo $\delta: C^{n-1}(G, M) \rightarrow C^{n}(G, M)$ é dado por

$$
(\delta f)\left(g_{1}, \ldots, g_{n}\right)=g_{1} f\left(g_{2}, \ldots, g_{n}\right)-f\left(g_{1} g_{2}, \ldots, g_{n}\right)+\cdots+(-1)^{n} f\left(g_{1}, \ldots, g_{n-1}\right) .
$$

Em particular, $\delta: C^{0}(G, M) \rightarrow C^{1}(G, M)$ é tal que $(\delta f)(g)=g f\lfloor]-f[]$.

1.5: Sejam $G$ um grupo e $M$ um $R G$-módulo. O grupo das derivaçōes de $G$ em $M$ é definido por

$$
\operatorname{Der}(G, M)=\{f: G \rightarrow M \mid f(g h)=g f(h)+f(g), \quad \forall g, h \in G\},
$$


e o subgrupo das derivaçōes principais por

$$
P(G, M)=\left\{d_{m}: G \rightarrow M, m \in M \mid d_{m}(g)=g m-m, \forall g \in G\right\}
$$

Observe que, no caso em que $R=\mathbf{Z}_{2}$, temos por 1.2 (a),

$$
P(G, M)=\left\{d_{m}: G \rightarrow M, m \in M \mid d_{m}(g)=g m+m, \forall g \in G\right\} .
$$

Usando a resolução bar obtem-se facilmente a seguinte interpretação de $H^{1}(G, M)$ em termos de derivaçôes e derivações principais:

1.6 Lema: Se $G$ é um grupo e $M$ é um $R G$-módulo, então

$$
H^{1}(G, M)=\frac{\operatorname{Der}(G, M)}{P(G, M)}
$$

$\mathrm{O}$ resultado abaixo nos diz que, na categoria de $\mathbf{Z}_{2} G$-módulos, os grupos de (co)homologia sobre $\mathbb{Z}_{2}$ e $\mathbb{Z}$ são equivalentes. Nós apresentamos aqui um esboço da prova deste resultado, visto que ele não se encontra em nossa referência básica [8].

1.7 Proposição: $S \epsilon M$ é um $\mathbb{Z}_{2} G$-módulo então temos um isomorfismo natural de $H^{*}(G ; M)$ com $M$ visto como $\mathbb{Z}_{2} G$-módulo $\epsilon H^{*}(G ; M)$ para $M$ considerado como $\mathbb{Z} G$-módulo. Analogamente para $H_{.}(G ; M)$.

Demonstração: Seja $F \rightarrow \mathbb{Z}$ uma resolução projetiva de $\mathbb{Z}$ sobre $\mathbb{Z} G$. Então $F^{\prime}=F \otimes_{Z} \mathbb{Z}_{2} \rightarrow \mathbb{Z} \otimes_{Z} \mathbb{Z}_{2}=\mathbb{Z}_{2}$ é uma $\mathbb{Z}_{2} G$-resolução projetiva de $\mathbf{Z}_{2}$ (cf. $[9$, p.6]). Agora nós temos isomorfismos naturais

$$
\begin{aligned}
& \phi: \operatorname{Hom}_{G}\left(F^{\prime}, M\right) \rightarrow \operatorname{Hom}_{\mathbf{Z} G}(F, M) \\
& \psi: F^{\prime} \otimes_{G} M \quad \rightarrow \quad F \otimes_{Z_{G} M}
\end{aligned}
$$

dados por $\phi(f)(x)=f(x \otimes 1), \quad \psi^{\prime}((x \otimes r) \otimes m)=x \otimes r m, \quad \forall f \in H_{o} m_{G}\left(F^{\prime}, M\right)$, $x \in F, \quad r \in \mathbb{Z}_{2}$ e $m \in M$; o que produz, levando em conta a definição 1.3 , o resultado desejado.

1.8 Interpretação Topológica: Finalizando esta secção veremos (na proposiçâo abaixo) que a (co)homologia de $G$ está relacionada com complexos $K(G, 1)$. 
Recordemos que se $Y$ é um $C W$-complexo então $Y$ é denominado complexo de Eilemberg-MacLane de tipo $(G, 1)$ ou simplesmente um complexo $K(G, 1)$ se $Y$ satisfaz as seguintes condiçôes:

(i) $Y$ é conexo

(ii) $\pi_{3}(Y)=G$

(iii) $O$ recobrimento universal de $Y$ é contráctil ( ou equivalentemente, $\pi_{i}(Y)=0$ para todo $\left.i \geq 2\right)$.

Dado um grupo $G$ é sempre possivel construir um complexo $K(G, 1)[35,2.10]$.

Também, dado um $\mathbb{Z} G$-módulo $M$ e $Y$ um complexo $K(G, 1)$ podemos construir o sistema de coeficientes locais $\mathcal{M}$ sobre $Y$ associado ao $\mathbf{Z} G$-módulo $M[36$, cap. VI].

Para a demonstraçào da proposiçào nos referimos $[8,11.4 .1]$ para o caso em que $M=R$ com a $G$-ação trivial e [15, teor. 10.7.14c] para o caso geral, sempre levando em consideração a proposição anterior.

1.9 Proposição: Sejam $G$ um grupo, $M$ um $R G$-módulo e $Y$ um complexo $K(G, 1)$. Entâao

(i) $H_{.}(G ; M) \simeq H_{.}(Y ; \mathcal{M}) \epsilon$,

(ii) $H^{*}(G ; M) \simeq H^{*}\left(Y^{\prime} ; \mathcal{M}\right)$.

onde $\mathcal{M}$ é o sistema de coeficientes locais sobre $Y$ associado ao $\mathbf{Z} G$-módulo $M$.

Note que se $M$ é um $R G$-módulo trivial (isto é, $g \cdot m=m, \forall g \in G$ ) então

$$
H_{.}(G ; M) \simeq H_{.}(Y, M) \quad \epsilon \quad H^{*}(G ; M) \simeq H^{*}(Y, M),
$$

em particular

$$
H_{*}(G)=H_{*}\left(Y^{\prime}\right) \quad \in \quad H^{*}(G)=H^{*}(Y) .
$$




\section{2. $H^{*}$ e $H$, como funtores do módulo coeficiente e funtores de duas variáveis}

2.1: Uma vez que $F \otimes_{R G}-$ e $H o m_{R G}(F,-)$ são funtores covariantes, é claro que $H_{.}(G ;-)$ e $H^{*}(G ;-)$ serão também funtores covariantes da categoria de $R G$-módulos na categoria dos grupos abelianos. Se $f: M \rightarrow M^{\prime}$ é um $R G$-homomorfismo a aplicação induzida $H_{*}(G ; f): H_{*}(G ; M) \rightarrow H_{*}\left(G ; M^{\prime}\right)\left(H^{*}(G ; f): H^{*}(G ; M) \rightarrow H^{*}\left(G ; M^{\prime}\right)\right)$ será denotada, caso não haja confusão, simplesmente por $f$. (resp. $f^{*}$ ).

$\mathrm{Na}$ proposição abaixo apresentamos algumas propriedades básicas dos funtores $H_{.}(G ;-)$ e $H^{*}(G ;-)$. Antes introduziremos algumas notaçöes.

Seja $M$ um $R G$-módulo. Denotemos por

$M^{G}=\{m \in M \mid g m=m, \forall g \in G\} \quad \mathbf{e}$,

$M_{G}=M /<g m-m ; g \in G, m \in M>$ (o quociente de $M$ pelo subgrupo aditivo gerado pelos elementos da forma $g m-m, m \in M, g \in G$ ).

Pode-se verificar, facilmente, que

$$
M^{G} \simeq \operatorname{Hom}_{R G}(R, M) \text { e } M_{G} \simeq R \otimes_{R G} M .
$$

2.2 Proposição:[8, III.6.1] Seja $M$ um $R G$-módulo:

(i) Existc um isomorfismo natural $H_{0}(G ; M) \simeq M_{G}$

(i)' Existe um isomorfismo natural $H^{0}(G ; M) \simeq M^{G}$

(ii) Para qualquer sequencia exata $0 \rightarrow M^{\prime} \stackrel{\text { 古}}{\rightarrow} M \stackrel{j}{\rightarrow} M^{n} \rightarrow 0$ de $R G$. módulos e qualquer inteiro n existe uma aplicação natural $\partial: H_{n}\left(G ; M^{\prime \prime}\right) \rightarrow H_{n-1}\left(G, M^{\prime}\right)$ tal que a sequência

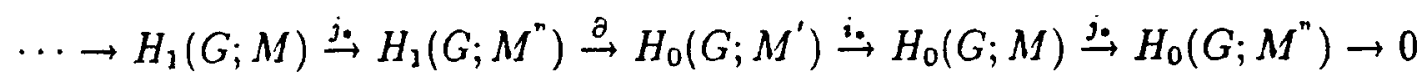

é exata.

(ii)' Para qualquer sequência exata como em (ii) $\epsilon$ qualquer inteiro $n$ existe uma aplicação natural $\delta: H^{n}\left(G ; M^{n}\right) \rightarrow H^{n+1}\left(G ; M^{\prime \prime}\right)$ tal que a sequência

$$
\left.0 \rightarrow H^{0}\left(G ; M^{\prime}\right) \stackrel{i_{0}}{\rightarrow} H^{0}(G ; M) \stackrel{j_{.}}{\rightarrow} H^{0}\left(G ; M^{\prime \prime}\right) \stackrel{6}{\rightarrow} H^{1} G ; M^{\prime}\right) \stackrel{i}{\rightarrow} H^{1}(G ; M) \rightarrow \cdots
$$


é exata.

Podemos mais geralmente ver $H$. e $H^{*}$ como funtor de duas variáveis como segue:

2.3: Vejamos $H$. como um funtor de duas variáveis. Seja $\mathcal{C}_{0}$ a seguinte categoria: Um objeto de $\mathcal{C}_{0}$ é um $\operatorname{par}(G, M)$ onde $G$ é um grupo e $M$ um $R G$-módulo; uma aplicação de $(G, M)$ em $\left(G^{\prime}, M^{\prime}\right)$ é um $\operatorname{par}(\alpha, f)$ onde $\alpha: G \rightarrow G^{\prime}$ é uma aplicação de grupos e $f: M \rightarrow M^{\prime}$ é uma aplicação de grupos abelianos tal que $f(g m)=\alpha(g) f(m)$, para $g \in G, m \in M$ (isto é, $f$ é um homomorfismo de $R G$-módulos se $M^{\prime}$ é considerado como $R G$-módulo via a (ver secção seguinte)). Dado $(\alpha, f)$ seja $F$ (resp. $F^{\prime}$ ) uma resolução projetiva de $R$ sobre $R G$ (resp. $R G^{\prime}$ ) e seja $\tau: F \rightarrow F^{\prime}$ uma aplicação de cadeja compativel com $\alpha$ como em [8, II.6], (isto é, $\tau(g x)=\alpha(g) \tau(x)$, para $g \in G$ e $x \in F$ ). Então existe uma aplicação de cadeia

$$
\tau \otimes f: F \otimes_{R G} M \rightarrow F^{\prime} \otimes_{R G} M^{\prime}
$$

e $\tau \otimes f$ induz uma aplicação bem definida

$$
(\alpha, f) .: H_{*}(G ; M) \rightarrow H_{*}\left(G^{\prime} ; M^{\prime}\right)
$$

Deste modo $H$. torna-se um funtor covariante sobre $\mathcal{C}_{0}$. No caso em que $M=M^{\prime}$ e $f=i d$, nós às vezes simplesmente escrevemos $a_{*}$ para $\left(\alpha, i d_{M^{\prime}}\right)_{*}: H_{*}\left(G ; M^{\prime}\right) \rightarrow$ $H_{*}\left(G^{\prime} ; M^{\prime}\right)$.

Note que a aplicação geral $(\alpha, f)$. pode ser escrita como a composta

$$
H_{*}(G ; M) \stackrel{H_{.}(G ; f)}{\rightarrow} H_{*}\left(G ; M^{\prime}\right) \stackrel{a_{*}}{\rightarrow} H_{*}\left(G^{\prime} ; M^{\prime}\right)
$$

onde $H_{*}(G ; f)$ faz sentido porque $f$ é uma aplicação de $R G$-módulos.

2.4: Para a cohomologia a situação é semelhante. Seja $\mathcal{C}_{1}$ a categoria com os mesmos objetos que $\mathcal{C}_{0}$, mas onde uma aplicaçâo de $(G, M)$ em $\left(G^{\prime}, M^{\prime}\right)$ é agora um par $\left(a: G \rightarrow G^{\prime}, f: M^{\prime} \rightarrow M\right)$. Como antes nós exigimos que $f$ seja uma aplicação de $R G$-módulos via $\alpha$, isto é, $f\left(\alpha(g) m^{\prime}\right)=g f\left(m^{\prime}\right)$ para $g \in G, m^{\prime} \in M^{\prime}$. Se $F$ e $F^{\prime}$ são 
resoluçōes para $G$ e $G^{\prime}$ e $\tau: F \rightarrow F^{\prime}$ é uma aplicação de cadeia compativel com $\alpha$, existe uma aplicação de cadeia

$$
\operatorname{Hom}(\tau, f): \operatorname{Hom}_{R G}\left(F^{\prime}, M^{\prime}\right) \rightarrow \operatorname{Hom}_{R G}(F, M)
$$

que induz

$$
\begin{aligned}
(\alpha, f)^{*}: H^{*}\left(G^{\prime} ; M^{\prime}\right) & \rightarrow H^{*}(G ; M) . \\
{[k] } & \rightarrow[f \circ k \circ \tau]
\end{aligned}
$$

Assim $H^{*}$ é um funtor contravariante sobre $\mathcal{C}_{2}$. No caso em que $M=M^{\prime}$, escrevemos $\alpha^{*}$ para $\left(\alpha, i d_{M^{\prime}}\right)_{*}$; note que a aplicação geral $(\alpha, f)^{*}$ é a composta

$$
H^{*}\left(G^{\prime} ; M^{\prime}\right) \stackrel{a^{*}}{\rightarrow} H^{*}\left(G ; M^{\prime}\right) \stackrel{H^{\bullet}(G ; \rho)}{\rightarrow} H^{*}(G ; M) .
$$

\section{Módulos Induzidos e Coinduzidos}

Seja $\alpha: \Lambda \rightarrow \Gamma$ um homomorfismo de anéis unitários. Então qualquer $\Gamma$-módulo (à esquerda) $N$ pode ser considerado como um $\Lambda$-módulo (à esquerda) via $\alpha$, definindo

$$
a . n=a(a) n, \quad \forall a \in \Lambda, \quad n \in \Lambda^{\prime} .
$$

Neste caso dizemos que $N$ é um $\Lambda$-módulo por restriçòes de escalares. (Vale construção análoga para módulos à direita).

Por outro lado, dado um $\Lambda$-módulo (à esquerda) $M$ podemos obter, a partir de $M$, dojs $\Gamma$-módulos (à esquerda) bastante relacionados com $M$ :

1. Considere, por restrição de escalares, $\Gamma$ como um $\Lambda$-módulo à direita, então podemos definir o produto tensorial $\Gamma \otimes_{\Lambda} M$. Este produto pode ser visto como $\Gamma$ - módulo (à esquerda) definindo $x \cdot\left(x^{\prime} \otimes m\right)=x x^{\prime} \otimes m, \forall x, x^{\prime} \in \Gamma$ e $m \in M$ (onde $x x^{\prime}$ é o produto em $\Gamma$ ). $\Gamma \otimes_{\Lambda} M$ com esta estrutura de $\Gamma$-módulos é denominado o módulo obtido de $M$ por estensão de escalares de $\Lambda$ à $\Gamma$ (via $\alpha$ ). 
2. Analogamente, considere, por restriçào de escalares, $\Gamma$ como um $\Lambda$-módulo à esquerda. Então podemos definir $\operatorname{Hom}_{\Lambda}(\Gamma, M)$. $H o m_{\Gamma}(\Gamma, M)$ pode ser visto como $\Gamma$-módulo (à esquerda) definindo $(x . f)\left(x^{\prime}\right)=f\left(x^{\prime} x\right), \quad \forall x, x^{\prime} \in \Gamma$ e $f \in$ $H_{o m}(\Gamma, M)$. Este $\Gamma$-módulo é denominado o módulo obtido de $M$ por co-extensão de escalares (via $\alpha$ ).

O lema a seguir será utilizado na construçâo 3.2 abaixo. Embora seja ele mentar, o demonstraremos aqui para familiarizar o leitor com as muitas açôes envolvidas.

3.1 Lema: Seja $M$ um $\Lambda$-módulo. Se $\Gamma \otimes_{\Lambda} M$ e $H o m_{\Lambda}(\Gamma, M)$ são os $\Gamma$-módulos obtidos de $M$ por extensão e co-extensâo de escalares, então as aplicaçöes naturais

$$
\begin{aligned}
& \text { 1) } i: M \rightarrow \Gamma \otimes_{\Lambda} M \text { dada por } i(m)=1 \otimes m, e \\
& \text { 2) } \pi: \operatorname{Hom}_{\Lambda}(\Gamma, M) \rightarrow M \text { dada por } \pi(f)=f(1) \quad(1 \in \Gamma)
\end{aligned}
$$

sâo $\Lambda$-homomorfismos se vemos os $\Gamma$-módulos $\Gamma \otimes_{\Lambda} M \in H_{o m}(\Gamma, M)$ como $\Lambda$-módulos por restriçâo de escalares via $\alpha$.

\section{Demonstração:}

1) $i(a m)=1 \otimes a m=1 . a \otimes m=1 \alpha(a) \otimes m=\alpha(a) 1 \otimes m=\alpha(a)(1 \otimes m)=$ $a . i(m)$, para todo $a \in \Lambda$ e $m \in M$.

2) $\pi(a . f)=\pi(a(a) f)=(a(a) f)(1)=f(1 a(a))=f(\alpha(a) 1)=f(a .1)=$ $a f(1)=a \pi(f)$, para todo $a \in \Lambda$ e $f \in \operatorname{Hom}_{\Lambda}(\Gamma, M)$.

3.2: $[8$, III.3.3 e 3.6] Sejam $\alpha: \Lambda \rightarrow \Gamma$ um homomorfismo de anéis (unitários), $M$ um $\Lambda$-módulo e $N$ um $\Gamma$-módulo. Entâo:

1. Dado uma aplicação de $\Lambda$-módulos $f: M \rightarrow N$ (onde $N$ é visto como $\Lambda$-módulo via $a$ ), existe uma única aplicação de $\Gamma$-módulos $\varphi: \Gamma \otimes_{\Lambda} M \rightarrow \Lambda^{\prime}$ tal que $\varphi \circ i=f$. A aplicação $\varphi$ é dada por $\varphi(x \otimes m)=x f(m)$.

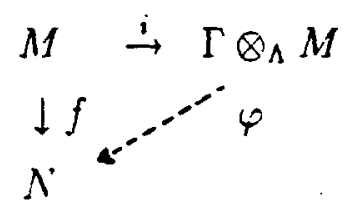


Assim obtemos um isomorfismo

$$
\begin{aligned}
\rho: \operatorname{Hom}_{\Lambda}(M, N) & \cong \operatorname{Hom}_{\Gamma}\left(\Gamma \otimes_{\Lambda} M, N\right) \\
f & \rightarrow \rho(f)=\varphi,
\end{aligned}
$$

com inverso dado por $\rho^{-1}(\varphi)=\varphi \circ i$.

2. Dada uma aplicação de $\Lambda$-módulos $f: N \rightarrow M$, existe uma única aplicação de $\Gamma$-módulos $\psi: N \rightarrow \operatorname{Hom}_{\Lambda}(\Gamma, M)$ tal que $\pi \circ \psi=f$. A aplicação $\psi$ é dada por $\psi(n)(a)=f(a n)$.

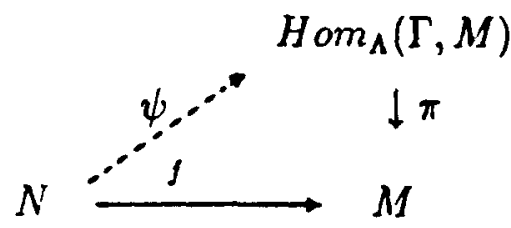

Assim obtemos um isomorfismo

$$
\begin{aligned}
\gamma: \operatorname{Hom}_{\Lambda}(N, M) & \stackrel{\sim}{\rightarrow} \operatorname{Hom}_{\Gamma}\left(N, \operatorname{Hom}_{\Lambda}(\Gamma, M)\right. \\
f & \rightarrow \gamma(f)=\psi
\end{aligned}
$$

com inverso dado por $\gamma^{-1}(\psi)=\pi \circ \psi$.

Sejam $G$ um grupo e $S$ um subgrupo de $G$. Nosso interesse é aplicar a teoria anterior para o homomorfismo de anéis $a: \Lambda=\mathbb{Z}_{2} S \rightarrow \Gamma=\mathbb{Z}_{2} G$, induzido da inclusão $S \subset G$.

Notação: Seja $N$ um $\mathbb{Z}_{2} G$-módulo.Denotaremos por $\quad R \in s_{S}^{G} N \quad$ o $\mathbb{Z}_{2} G$ módulo obtido de $N$ por restrição de escalares de $G$ a $S$ (via $a: \mathbb{Z}_{2} S \rightarrow \mathbb{Z}_{2} G$ ).

3.3 Definição: Scja $M$ um $\mathbb{Z}_{2} S$-módulo. $O \mathbb{Z}_{2} G$-módulo obtido de $M$ por extensâo (resp. co-extensão) de escalares de $\mathbb{Z}_{2} S$ a $\mathbb{Z}_{2} G$ via a é denominado indução (resp. co-indusãa) de $S$ em $G$.

Escreveremos:

$$
\begin{aligned}
\operatorname{Ind} d_{S}^{G} M & =\mathbb{Z}_{2} G \otimes_{S} M . \\
\operatorname{Coind} d_{S}^{G} M & =\operatorname{Hom}_{S}\left(\mathbb{Z}_{2} G, M\right) .
\end{aligned}
$$

Observemos que em $\ln d_{S}^{G} M, \mathbb{Z}_{2} G$ é visto como $\mathbb{Z}_{2} S$-módulo à direita com a $S$-ação $g . s=g \alpha(s)=g s$ ( multiplicaçào à direita), e em $\operatorname{Coind} d_{S}^{G} M, \mathbf{Z}_{2} G$ é visto como $\mathbf{Z}_{2} S$-módulo com a $S$-açâo multiplicação à esquerda $(s \cdot g=s g)$. 
Ind $d_{S}^{G} M$ é visto como $Z_{2} G$-módulo com $G$-açâo dada por $g \cdot\left(g^{\prime} \otimes m\right)=$ $g g^{\prime} \otimes m, \forall g, g^{\prime} \in G$ e $m \in M$; e $C o i n d S_{S}^{G} M$ é visto como $Z_{2} G$-módulo com a $G$-ação $(g . f)\left(g^{\prime}\right)=f\left(g^{\prime} g\right), \quad \forall g, g^{\prime} \in G, \quad f \in H_{\text {om }}\left(\mathbf{Z}_{2} G, M\right)$.

Os módulos da forma $\operatorname{Ind} d_{\{1\}}^{G} M$ e $\operatorname{Coind}_{\{1\}}^{G} M$ são denominados, respectivamente, módulos induzidos e co-induzidos.

Denotaremos o módulo coinduzido $\operatorname{Coin}_{(1)}^{G} \mathbb{Z}_{2}$ por $\overline{\mathbb{Z}_{2} G}$.

3.4 Observaçōes: (a) Considerando os $\mathbf{Z}_{2} S$-homomorfismos do lema 3.1, pode-se facilmente verificar que $i: M \rightarrow I_{n} d_{S}^{G} M(m \rightarrow 1 \otimes m)$ é um $\mathbf{Z}_{2} S$. monomorfismo, e $\pi: \operatorname{Coind}_{S}^{G} M \rightarrow M \quad(f \rightarrow f(1))$, é um $\mathbb{Z}_{2} S$-epimorfismo.

(b) $\mathbb{Z}_{2} G$ é um $\mathbb{Z}_{2} S$-módulo livre, como base podemos tomar qualquer conjunto $E$ de representantes para as classes laterais $g S$. Assim $\mathbf{Z}_{2} G=\bigoplus_{g \in E} g \mathbf{Z}_{2} S$.

Segue de (a) que $M$ é canonicamente $\mathbf{Z}_{2} S$-isomorfo a $1 \otimes M$, portanto podemos identificar $M$ com $1 \otimes M$.

Por outro lado, segue de (b), que $\mathbb{Z}_{2} G \otimes S M$ como grupo abeliano admite a decomposição

$$
\mathbb{Z}_{2} G \otimes S M=\bigoplus_{g \in G / S} g \otimes M
$$

onde $g \otimes M=\{g \otimes m, m \in M\}$. (Por um abuso de notaçào estamos indicando um conjunto de representantes para as classes laterais $g S$ por $G / S)$.

Como $g(1 \otimes m)=g \otimes m$, então $g \otimes M$ é simplesmente o transformado de $1 \otimes m$ pela açâo de $G$. Logo podemos identificar $g \otimes m=g(1 \otimes m)$ com $g M$. Assim temos:

3.5 Proposição: $[8,111.5 .1]$ Seja $M$ um $\mathbb{Z}_{2} S$-módulo.

(i) $O \mathbb{Z}_{2} G$-módulo Inda $M$ contém $M$ como $\mathbb{Z}_{2} S$ - módulo e admite uma decomposiçâo em soma dirte

$$
\operatorname{Ind} d_{S}^{G} M=\bigoplus_{g \in G / S} g M
$$

onde gM é o transformado de $M$ pela ação de $G$.

Analogamente, 
(ii) Coind ${ }_{S}^{G} M$ admite uma decomposição em produto direto, isto é, existe uma familia de sobrejeçōes $\pi_{g}:$ Coind ${ }_{S}^{G} M \rightarrow g M, g \in G / S$ tal que a aplicação correspondente

$$
\operatorname{Coind}_{S}^{G} M \rightarrow \prod_{\rho \in G / S} g M
$$

é um isomorfismo.

Seja $M$ um $\mathbf{Z}_{2} S$-módulo. Consideremos a aplicação

$$
\varphi_{0}: M \rightarrow \operatorname{Coind}_{S}^{G} M \text { onde } m \rightarrow \varphi_{0}(m) \mid \varphi_{0}(m)(g)= \begin{cases}g m & \text { se } g \in S \\ 0 & \text { c.c. }\end{cases}
$$

Então $\varphi_{0}$ é claramente um $\mathbb{Z}_{2} S$-homomorfismo, pois

$$
\begin{gathered}
\varphi_{0}(s m)(g)= \begin{cases}g(s m)=g s m & \text { se } g \in S, \\
0 & \text { c.c. }\end{cases} \\
s \varphi_{0}(m)(g)=\varphi_{0}(m)(g s)= \begin{cases}g s m & \text { se } g s \in S, \\
0 & \text { c. c. }\end{cases}
\end{gathered}
$$

e, como $s \in S$, temos que $g s \in S$ se e somente se $g \in S$.

Considerando em 3.2 (1), $N=\operatorname{Coind}_{S}^{G} M$ e $f=\varphi_{0}$, existe uma única aplicação de $\mathbb{Z}_{2} G$-módulos $\varphi: \operatorname{Ind} d_{S}^{G} M \rightarrow \operatorname{Coind}_{S}^{G} M$ tal que $\varphi \circ i=\varphi_{0}$. $\varphi$ é dada por $\varphi\left(g_{0} \otimes m\right)=g_{0} \varphi_{0}(m)$ e portanto,

$$
\varphi\left(g_{0} \otimes m\right)(g)=\left(g_{0} \varphi_{0}(m)(g)=\varphi_{0}(m)\left(g g_{0}\right)= \begin{cases}g g_{0} m & \text { se } g g_{0} \in S \\ 0 & \text { c.c. }\end{cases}\right.
$$

$O$ resultado seguinte nos diz que podemos sempre ver $\ln d_{S}^{G} M$ como um submódulo de $\operatorname{Coind}_{S}^{G} M$ (via a aplicaçâo $\varphi$ ).

3.6 Proposição: $[8,111.5 .9]$ Seja $M$ um $\mathbb{Z}_{2} S$-módulo.

(i) $A \mathbb{Z}_{2} G$-aplicação $\varphi: \operatorname{Ind}_{S}^{G} M \rightarrow \operatorname{Coind}_{S}^{G} M$ definida acima é um $\mathbb{Z}_{2} G$ monomorfismo, e se escrevermos Inds ${ }_{S}^{G} M$ como uma soma direta $\epsilon$ Coind ${ }_{S}^{G} M$ como um produto direto de cópias de $M$ então $\varphi$ pode ser identificada com a inclusâo canônica da soma no produto. 
(ii) $\operatorname{Se}[G: S]<\infty$ então $\varphi: \operatorname{Ind}_{S}^{G} M \rightarrow \operatorname{Coind}_{S}^{G} M$ é um $\mathbf{Z}_{2} G$-isomorfismo.

3.7 Observação: Em particular podemos considerar (através da $\varphi$ ), $\mathbf{Z}_{2} G \simeq \operatorname{Ind} d_{(1)}^{G} \mathbb{Z}_{2}$ como um submódulo do módulo coinduzido $\overline{\mathbf{Z}_{2} G}=\operatorname{Coind}_{\{1}^{G} \mathbf{Z}_{2}$. Neste caso $\varphi$ é dada por

$$
\varphi\left(g_{0}\right)(g)=\varphi\left(g_{0} \otimes 1\right)(g)=\left\{\begin{array}{ll}
g g_{0} & \text { se } g g_{0} \in\{1\} \\
0 & \text { c. c. }
\end{array}= \begin{cases}1 & \text { se } g=g_{0}^{-1} \\
0 & \text { c. c. }\end{cases}\right.
$$

para todo $g_{0}, g \in G$.

As propriedades seguintes são consequências de 3.2 e 3.6 respectivamente. As apresentaremos aqui para facilitar futuras referencias.

3.8 Lema: $S \epsilon S$ e $T$ sâo subgrupos de $G$ satisfazendo $G \supset T \supset S$ e $M$ em $\mathbf{Z}_{2} S$-módulo então:

(a) Existe um $\mathbf{Z}_{2} G$-isomorfismo natural Coind ${ }_{T}^{G} \operatorname{Coind} d_{S}^{T} M \stackrel{\psi}{\rightarrow}$ Coind $S_{S}^{G} M$.

(b) Existe um $\mathbb{Z}_{2}$ G-monomorfismo natural Ind ${ }_{T}^{G}$ Coind ${ }_{S}^{T} M \stackrel{\varphi}{\rightleftharpoons}$ $\operatorname{Coind}_{T}^{G} \operatorname{Coind}_{S}^{T} M$.

Demonstração: (a) Segue de 3.2 (2) tomando $N=\mathbb{Z}_{2} G, \Gamma=\mathbb{Z}_{2} S, \Lambda=$ $\mathbb{Z}_{2} T$ e $\psi=\gamma^{-1}$.

(b) Basta considerar, em 3.6, $T$ no lugar de $S$ e o $\mathbb{Z}_{2} T$-módulo $C o i n d_{S}^{T} M$ no lugar de $M$.

Antes de apresentarmos o próximo resultado, que será muito útil nos capítulos seguintes, observamos que, dado um subgrupo $T$ de $G$ e um $\mathbb{Z}_{2} T$-módulo $M$, o somando $g M$ de $I n d_{T}^{G} M$ é fechado sob a açâo de $T^{g}:=g T g^{-1}$ pois $g t g^{-1} \cdot(g \otimes m)=g t g^{-1} g \otimes m=$ $g t \otimes m=g \otimes t m$ (para todo $t \in T$ e $m \in M$ ).

Assim $g M$ é um $\mathbb{Z}_{2} T^{g}$-módulo. Em particular, se $T$ é normal em $G$ entâo $g M$ é um $\mathbf{Z}_{2} T$-módulo.

3.9 Proposição (Fórnula de Mackey): [8, III.5.6(b)] Sejam $T$ e $S$ 
subgrupos de $G$. Seja $E$ um conjunto de representantes para as classes duplas $S g T$. Para qualquer $\mathbf{Z}_{2} T$-módulo $M$ existe um $\mathbf{Z}_{2} S$-isomorfismo

$$
\operatorname{Res}_{S}^{G} \operatorname{Ind} d_{T}^{G} M \simeq \bigoplus_{\theta \in E} I n d_{S \cap T \theta}^{S} \operatorname{Res} s_{S \cap T \theta}^{T \theta} g M .
$$

Em particular, se $T$ é normal em $G$, entào existe um $\mathbf{Z}_{2} T$-isomorfismo

$$
R e s_{T}^{G} \ln d_{T}^{G} M \simeq \bigoplus_{g \in G / T} g M .
$$

A seguir, apresentaremos dois lemas, que serão frequentemente usados neste trabalho. Como em nossa referência básica [8] eles estão propostos como exercicios, nós os demonstraremos aqui.

3.10 Lema: Se $M$ é um $\mathbb{Z}_{2} S$-módulo e $N$ um $\mathbb{Z}_{2} G$-módulo então temos um $\mathbb{Z}_{2} G$-isomorfismo

$$
N \otimes \operatorname{In} d_{S}^{G} M \simeq \operatorname{In} d_{S}^{G}\left(R \in s_{S}^{G} N \otimes M\right),
$$

onde o produto tensorial à esquerda tem a G-açâo diagonal e o da direita a $S$-ação diagonal.

Demonstração: Considere a aplicaçào

$$
\begin{aligned}
k: N \otimes\left(\mathbb{Z}_{2} G \otimes S M\right) & \rightarrow \mathbb{Z}_{2} G \otimes_{S}\left(\operatorname{Res}_{S}^{G} N \otimes M\right), \\
n \otimes(g \otimes m) & \rightarrow g \otimes\left(g^{-1} n \otimes m\right),
\end{aligned}
$$

e estendida por linearidade. Para qualquer $s \in S$ temos

$$
\begin{aligned}
k(n \otimes(g \otimes s m)) & =g \otimes\left(g^{-1} n \otimes s m\right)=g \otimes s\left(s^{-1} g^{-1} n \otimes m\right)= \\
& =g s \otimes\left((s g)^{-1} n \otimes m\right)=k(n \otimes(g s \otimes m)),
\end{aligned}
$$

e portanto $k$ está bem definida. Também, para qualquer $g_{0} \in G$, temos

$$
\begin{aligned}
k\left(g_{0}(n \otimes(g \otimes m))\right) & =k\left(g_{0} n \otimes\left(g_{0} g \otimes m\right)\right)=g_{0} g \otimes\left(\left(g_{0} g\right)^{-1} g_{0} n \otimes m\right)= \\
& =g_{0} g \otimes\left(g^{-1} g_{0}^{-1} g_{0} n \otimes m\right)=g_{0}\left(g \otimes\left(g^{-1} n \otimes m\right)\right)= \\
& =g_{0} k(n \otimes(g \otimes m)),
\end{aligned}
$$

e assim $k$ é um $\mathbb{Z}_{2} G$-homomorfismo. Agora, é fácil ver que a aplicaçâo

$$
\begin{aligned}
& l: \mathbb{Z}_{2} G \otimes_{S}\left(\operatorname{Res}_{S}^{G} N \otimes M\right) \rightarrow N \otimes\left(\mathbb{Z}_{2} G \otimes_{S} M\right) \\
& g \otimes(n \otimes m) \rightarrow g n \otimes(g \otimes m)
\end{aligned}
$$


é a inversa de $k$. Portanto $k$ é um $\mathbf{Z}_{2} G$-isomorfismo, como desejado.

ㅁ.

3.11 Lema: $\operatorname{Se}[G: S]=\infty$ entào

$$
H^{0}\left(G ; \operatorname{In} d_{S}^{G} M\right)=\left(\ln d_{S}^{G} M\right)^{G}=0,
$$

para todo $\mathbf{Z}_{2} S$-módulo $M$.

Demonstração: Suponhamos por absurdo que $\left(\ln d_{S}^{G} M\right)^{G} \neq 0$, isto é, que existe $u \in \operatorname{Ind}_{S}^{G} M, u \neq 0$, tal que $g u=u$ para todo $g \in G$.

Sabemos por 3.6 (i), que $I n d_{S}^{G} M=\bigoplus_{g \in E} g M$, onde $E$ é um conjunto de representantes para $G / S$ (com $e \in E$, onde $e$ é o elemento neutro de $G$ ). Assim, $u=g_{1} m_{1}+\ldots+g_{k} m_{k}$, com $g_{i} \in E, g_{i} m_{i} \neq 0$ e $g_{i} m_{i} \neq g_{j} m_{j}$ para todo $i \neq j$ (pois $\left.\bigcap_{g \in E} g M=0\right)$.

Seja $g \in G$. Como a aplicação $\operatorname{Ind} d_{S}^{G} M \rightarrow \operatorname{Ind} d_{S}^{G} M(v \rightarrow g v)$ é uma bijeção, segue que $g g_{i} m_{i} \neq g g_{j} m_{j}$ se $i \neq j$.

Afirmação: $m_{i}=m_{j}=m$, para todo $i \neq j$.

De fato, para cada $i, j$, seja $g:=g_{i} g_{j}^{-1}$. Como $g u=u$, temos que $g g_{1} m_{1}+$ $\ldots+g g_{i} m_{i}+\ldots+g_{i} m_{j}+\ldots+g g_{k} m_{k}=g_{1} m_{1}+\ldots+g_{k} m_{k}$. Agora, $g_{i} m_{j}=g g_{j} m_{j} \neq g g_{r} m_{r}$ se $j \neq r, g_{i} m_{j}$ é não nulo, e $g_{i} m_{j} \neq g_{r} m_{r}$ se $r \neq i$ (pois $g_{1}, g_{r} \in E$ e $\bigcap_{g \in E} g M=0$ ). Logo a única possibilidade é que $g_{i} m_{j}=g_{i} m_{i}$. Daj, $m_{j}=m_{i}$.

Consequentemente, temos que $u=g_{1} m+\ldots+g_{k} m$. Como por hipótese $[G: S]=\infty$, existe $g_{0} \in E, g_{0} \notin\left\{g_{1}, \ldots, g_{k}\right\}$. Seja $g:=g_{0} g_{1}^{-1}$. Do fato que $g u=u$ segue que $g g_{1} m+\ldots+g g_{k} m=g_{1} m+\ldots+g_{k} m$ e dai, como $g g_{1} m \neq g g_{i} m$ se $i \neq 1$ e $g g_{1} m \neq 0$, deve existir algum $j \in\{1, \ldots, k\}$ tal que $g g_{1} m=g_{j} m$. Logo $g_{j}^{-1} g g_{1} m=e m \neq 0 \quad$ e então $\quad g_{j}^{-3} g g_{1} \in S \quad$ (pois $e \in E$ e $\bigcap_{g \in E} g M=0$ ). De onde segue, levando em conta que $g=g_{0} g_{1}^{-1}$, que $g_{j}^{-1} g_{0} \in S$ para algum $j$, mas isto contradiz o fato que $g_{0} \in E-\left\{g_{1}, \ldots, g_{k}\right\}$. Logo nossa suposição inicial é falsa, e portanto $\left(\operatorname{Ind} d_{S}^{G} M\right)^{G}=0$.

Agora, se $S$ é um subgrupo de $G$, consideremos o $\mathbf{Z}_{2}$-módulo livre gerado 
pelas classes laterais $g S, g \in G$, que denotaremos por $\mathbf{Z}_{2}(G / S)$.

Existe uma açâo natural de $G$ em $\mathbb{Z}_{2}(G / S)$ induzida pela multiplicação à esquerda $\left(g_{0}(g S)=g_{0} g S\right)$. Daí, $\mathbb{Z}_{2}(G / S)$ tem uma estrutura de $\mathbb{Z}_{2} G$-módulo à esquerda (cf. $1.2(\mathrm{a}))$.

Logo, se $N$ é um $\mathbf{Z}_{2} G$-módulo, entào podemos dar a $\mathbf{Z}_{2}(G / S) \otimes N$ e $\operatorname{Hom}\left(\mathbb{Z}_{2}(G / S), N\right)$ a estrutura de $\mathbb{Z}_{2} G$-módulo diagonal, como em 1.2 (c).

Sob estas consideraçôes temos:

3.12 Proposição: Sejam $G$ um grupo, $S$ um subgrupo de $G$ e $N$ um $\mathbf{Z}_{2} G$ módulo. Então temos $\mathbf{Z}_{2} G$-isomorfismos naturais

$$
\begin{aligned}
& \mathbb{Z}_{2}(G / S) \otimes N \stackrel{u}{\rightarrow} \quad \operatorname{Ind} \operatorname{Res}_{S}^{G} N, \\
& \operatorname{Hom}\left(\mathbb{Z}_{2}(G / S), N\right) \stackrel{\sim}{\rightarrow} \operatorname{Coind}_{S}^{G} \operatorname{Res}_{S}^{G} N,
\end{aligned}
$$

onde $\mathbb{Z}_{2}(G / S) \otimes N \in \operatorname{Hom}\left(\mathbb{Z}_{2}(G / S), N\right)$ sâo vistos como $\mathbb{Z}_{2} G$-módulos como acima.

Demonstração: A aplicação $u$ é definida por $u(g S \otimes n)=g \otimes g^{-1} n$, $g \in G, n \in N$ e estendida por linearidade. $\dot{E}$ fácil ver que $u$ está bem definida e é um $\mathbf{Z}_{2} G$-homomorfismo. A inversa de $u$ é dada por $g \otimes n \rightarrow g S \otimes g n$.

Analogamente, " é definida por $v(f)(g)=g f\left(g^{-1} S\right), \quad g \in G$ e $f \in \operatorname{Hom}\left(\mathbb{Z}_{2}(G / S), N\right)$, e sua inversa $v^{\prime}$ por $v^{\prime}(h)(g S)=g h\left(g^{-1}\right), g \in G$, $h \in \operatorname{Hom}_{S}\left(\mathbb{Z}_{2} G, r \in s_{S}^{G} N\right)$.

3.13 Observaçōes: (a) Se considerarmos no resultado anterior $N=\mathbf{Z}_{2}$ entào temos os $\mathbb{Z}_{2} G$-isomorfismos naturais

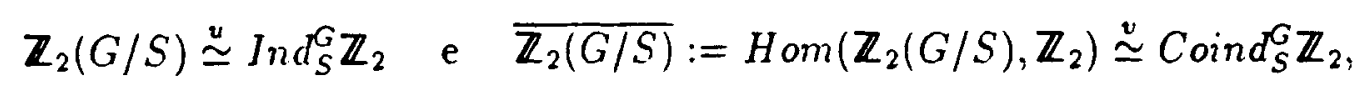

e portanto, por 3.6, obtemos o $\mathbb{Z}_{2} G$-monomorfismo $\mathbb{Z}_{2}(G / S) \stackrel{4}{\rightarrow} \overline{\mathbb{Z}_{2}(G / S)}$. Esta "inclusão" tem, como em $3 . \bar{T}$, a forma simples

$$
\varphi\left(g_{0} S\right)(g S)= \begin{cases}1 & \text { se } g^{-1} S=g_{0} S\left(\Leftrightarrow g g_{0} \in S\right) \\ 0 & \text { c. c. }\end{cases}
$$

(b) Se, agora, considerarmos $S=\{1\}$ e $N^{\prime}$ um $\mathbf{Z}_{2} G$-módulo qualquer, 
então temos os $\mathbb{Z}_{2} G$-isomorfismos

$$
\mathbb{Z}_{2} G \otimes N \stackrel{\sim}{\rightarrow} \operatorname{Ind} d_{\{1\}}^{G} N \quad \text { e } \quad \operatorname{Hom}\left(\mathbb{Z}_{2} G, N\right) \stackrel{\bullet}{\rightarrow} \operatorname{Coind}_{(1)}^{G} N
$$

onde os módulos da esquerda são vistos como $\mathbf{Z}_{2} G$-módulo com a $G$-ação diagonal. Note ainda que se $N$ é um $\mathbf{Z}_{2} G$-módulo trivial (ex: $N=\mathbf{Z}_{2}$ ) então $v$ tem a forma $v(g)(g)=$ $f\left(g^{-1}\right), \forall g \in G$.

$O$ resultado abaixo é também bastante utilizado nos próximos capítulos. Ele dá a relação entre a (co)homologia de um grupo e de um subgrupo.

3.14 Proposição (Lema de Shapiro): [8, IlI.6.2] Se $S$ é um subgrupo de $G$ e $M$ é um $\mathbb{Z}_{2} S$-módulo então

$$
H_{*}(S ; M) \simeq H_{*}\left(G ; \operatorname{Ind} d_{S}^{G} M\right) \quad \epsilon \quad H^{*}(S ; M) \simeq H^{*}\left(G ; \operatorname{Coind}_{S}^{G} M\right)
$$

Pode se verificar que os isomorfismos do lema de Shapiro são dados por

$$
H_{*}(S ; M) \stackrel{(a, i) \cdot}{\rightarrow} H_{*}\left(G ; \operatorname{In} d_{S}^{G} M\right) \quad \text { e } \quad H^{*}(S ; M) \stackrel{(a, \pi)^{\bullet}}{\rightarrow} H^{*}\left(G ; \operatorname{Coind}_{S}^{G} M\right)
$$

onde $(\alpha, i)=$ e $(\alpha, \pi)^{*}$ sào (cf. 2.3 e 2.4) as aplicaçòes induzidas, respectivamente, de

$$
\left(\alpha: S \rightarrow G, \quad i: M \rightarrow \operatorname{Ind}_{S}^{G} M\right) \text { e }, \quad\left(\alpha: S \rightarrow G, \pi: \operatorname{Coind}_{S}^{G} M \rightarrow M\right),
$$

com a $S \rightarrow G$ a inclusào, e $i$ e $\pi$ as $\mathbb{Z}_{2} S$-aplicaçòes dadas em 3.4.

3.15: Seja $G$ um grupo. Considere $P G=\{A \mid A \subseteq G\}$ o conjunto das partes de $G$. $\quad P G$ com a operaçào diferença simétrica $A+B:=A-B \cup B-A=$ $A \cap B^{C} \cup B \cap A^{C}$, é um grupo abeliano, em que todo elemento tem ordem 2 (portanto um $\mathbb{Z}_{2^{-}}$módulo). Dai, considerando a $G$-ação (multiplicaçâo à esquerda) $G \times P G \rightarrow P G$ $((g, A) \rightarrow g \cdot A=\{g \cdot a \mid a \in A\})$, podemos dar a $P G$ uma estrutura de $\mathbb{Z}_{2} G$-módulo (cf. $1.2(\mathrm{a}))$.

É fácil ver que a aplicaçào

$$
\begin{aligned}
\rho: \overline{\mathbb{Z}_{2} G}=\operatorname{Coind}_{\{1\}}^{G} \mathbb{Z}_{2} & \rightarrow P G \\
f & \rightarrow\left\{g \in G \mid f\left(g^{-1}\right)=1\right\}
\end{aligned}
$$


é um $\mathbf{Z}_{2} G$-isomorfismo. Ela é invariante pela ação pois, dado $g_{0} \in G$, temos:

$$
\begin{aligned}
\rho\left(g_{0} . f\right) & =\left\{g_{1} \in G \mid\left(g_{0} \cdot f\right)\left(g_{1}^{-1}\right)=1\right\} \\
& \left.=\left\{g_{1} \in G \mid f\left(g_{1}^{-1} g_{0}\right)=1\right\} \quad \text { (pela açào do Coind }{ }_{\{1\}}^{G} \mathbb{Z}_{2}\right) \\
& =\left\{g_{1} \in G \mid f\left(\left(g_{0}^{-1} g_{1}\right)^{-1}\right)=1\right\} \\
& \left.=\left\{g_{0} g \in G \mid f\left(g^{-1}\right)=1\right\} \quad \text { (considerando } g=g_{0}^{-1} g_{1}\right) \\
& =g_{0} \cdot\left\{g \in G \mid f\left(g^{-1}\right)=1\right\} \\
& =g_{0} . \rho(f) .
\end{aligned}
$$

A inversa de $\rho$ é dada por $P G \rightarrow \operatorname{Ind} d_{\{1\}}^{G} \mathbb{Z}_{2}\left(A \rightarrow f_{A}\right)$ onde,

$$
f_{A}(g)= \begin{cases}1 & \text { se } g^{-1} \in A \\ 0 & \text { c. c. }\end{cases}
$$

Levando em conta estes fatos tem-se, como consequéncia da proposição anterior, o seguinte resultado.

\subsection{Corolário:}

$$
H^{j}(G ; P G)= \begin{cases}\mathbb{Z}_{2} & \text { se } j=0 \\ 0 & \text { se } j \neq 0\end{cases}
$$

Finalizando esta seção, observamos que se $M 1$ é um $\mathbb{Z}_{2} G$-módulo (mais do que um $\mathbb{Z}_{2} S$-módulo), então podemos usar 3.12 e obter a seguinte versão do lema de Shapiro.

3.17 Proposição: Se $S$ é um subgrupo de $G \in M$ é um $\mathbb{Z}_{2} G$-módulo, então

$$
H_{*}(S ; M) \simeq H_{*}\left(G ; \mathbb{Z}_{2}(G / S) \otimes M\right), \quad \text { є } H^{*}(S ; M) \simeq H^{*}\left(G ; H \operatorname{Hom}\left(\mathbb{Z}_{2}(G / S), M\right)\right.
$$

onde $\mathbb{Z}_{2}(G / S) \& M \quad \epsilon \quad H o m\left(\mathbb{Z}_{2}(G / S), M\right)$ sào vistos como $\mathbb{Z}_{2} G$-módulos com a $G$-açâo diagonal.

Nota: Para sermos rigorosos deveríamos escrever na proposiçâo anterior $H_{*}\left(S ; R \epsilon s_{S}^{G} M\right)$ e $H^{*}\left(S ; R \epsilon s_{S}^{G} M\right)$. 


\section{4. $H^{1}(G ; M)$ e extensões de grupos.}

Esta seç̧ão será útil no capítulo Il de nosso trabalho, onde apresentamos uma "generalização" (para o caso relativo), do resultado 4.3 abaixo. Tal seç̧âo é baseada em [8, cap. IV]. Lá o autor apresenta também uma interpretaçào de $H^{2}$ e $H^{3}$ em termos de extensões de grupos, nós estamos interessados somente no caso $H^{1}$. Também ele trabalha sobre $R=\mathbf{Z}$, aqui adaptaremos ao caso $R=\mathbb{Z}_{2}$.

Inicialmente, observamos que uma extensão de um grupo $G$ por um grupo abeliano $M$

$$
0 \rightarrow M \stackrel{i}{\rightarrow} E \stackrel{\pi}{\rightarrow} G \rightarrow 1
$$

dá origem à uma açào de $G$ sobre $M$ (Aqui, estamos denotando o elemento neutro de $G$ por 1). Mais precisamente, dados $g \in G$ e $m \in M$, seja $x \in E$ tal que $\pi(x)=g$. Note que $x i(m) x^{-1} \in \operatorname{Im} i=$ Ker $\pi$ pois $\pi\left(x i(m) \cdot x^{-1}\right)=\pi(x) \pi(i(m)) \pi(x)^{-1}=\pi(x) \cdot 1 \cdot \pi(x)^{-1}=1$. Então definimos

$$
g . m=i^{-1}\left(x i(m) x^{-1}\right) .
$$

Fixemos agora um $Z_{2} G$-módulo $M$. Seja

$$
0 \rightarrow M \stackrel{i}{\rightarrow} E \stackrel{\pi}{\rightarrow} G \rightarrow 1
$$

uma extensão cuja ação de $G$ em $M$. obtida como em (1). coincide com a $G$-ação proveniente do fato de $M$ ser um $\mathbb{Z}_{2} G$-módulo.

Dizemos que (2) é uma extensấo cindida se existe un homomorfismo $l: G \rightarrow E$ tal que $\pi \circ l=i d_{G}$, e um tal homomorfismo é chamado um levantamento da extensâo.

Dois levantamentos $l_{1}$ e $l_{2}$ sào ditos $M$-conjugados se existe um elemento $m \in M$ tal que $l_{1}(g)=i(m) l_{2}(g) i(m)^{-1}$ para todo $g \in G$. (Isto da origem a uma relaçâo de equivalencia e portanto podemos falar em classes de equivalência $[l])$.

4.1 Exemplo: Seja $M$ um $\mathbb{Z}_{2} G$-módulo. Consideremos o grupo $E=M \times G$ com a operaçâo dada por

$$
\left(m_{1}, g_{1}\right) *\left(m_{2}, g_{2}\right)=\left(m_{1}+g_{1} \cdot m_{2} \cdot g_{1} g_{2}\right)
$$


Este grupo é denominado o produto semi-direto de $M$ por $G$ e é denotado por $M \rtimes G$.

A sequência $\quad 0 \rightarrow M \stackrel{i^{\prime}}{\rightarrow} M>G \stackrel{\pi^{\prime}}{\rightarrow} G \rightarrow 1$ onde $i^{\prime}(m):=(m, 1)$ e $\pi^{\prime}(m, g):=g$ é uma extensão cindida pois, por exemplo, $l: G \rightarrow M \rtimes G(g \rightarrow(0, g))$ é um levantamento para esta extensão.

O resultado seguinte nos diz que "a menos de equivalência", todas as extensōes cindidas são como no exemplo acima.

4.2 Proposição: [8, IV.2.1] As seguintes condiçōes sobre a extensão (2) sào equivalentes:

(a) (2) é cindida.

(b) (2) é equivalente à extensão $0 \rightarrow M \stackrel{i^{\prime}}{\rightarrow} M \rtimes G \stackrel{\pi^{\prime}}{\rightarrow} G \rightarrow 1$, isto é, existe uma aplicaçâo $k: E \rightarrow M \rtimes G$ (isomorfismo) tal que o diagrama

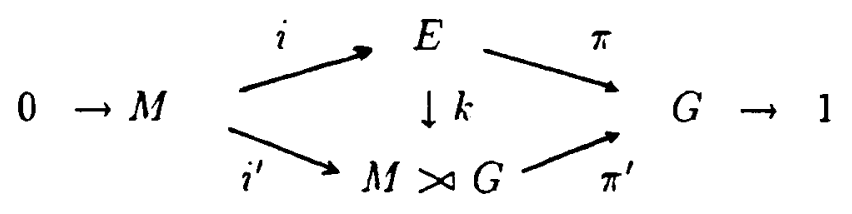

comuta.

Finalmente, temos a seguinte interpretaçào de $H^{1}(G ; M)$.

4.3 Proposição: Para qualquer $\mathbb{Z}_{2} G$-módulo $M$, as classes de $M$-conjugação [l] de levantamentos $l: G \rightarrow M \rtimes G$, da extensâo cindida $\quad 0 \rightarrow M \stackrel{i^{\prime}}{\rightarrow} M \rtimes G \stackrel{\pi^{\prime}}{\rightarrow} G \rightarrow 1$ estâo em correspondência biunívoca com os elementos de $H^{1}(G ; M)$.

Demonstração: Por simplicidade denotaremos o conjunto das classes de $M$-conjuga çào por $\mathcal{L}$. Sabemos por 1.6 que $H^{1}(G ; M)=\frac{\operatorname{Dcr}(G, M)}{P(G, M)}$.

Agora, os levantamentos $l: G \rightarrow M \rtimes G$ estào em correspondência biunivoca com as derivaçôes $d: G \rightarrow M$, pois um homomorfismo $l: G \rightarrow M \rtimes G$ que satisfaz $\pi \circ l=i d_{G}$ tem a forma $l(g)=(d(g), g)$, onde $d: G \rightarrow M$. Temos ainda $l(g) l(h)=l(g h) \Leftrightarrow(d(g)+g d(h), g h)=(d(g h), g h) \Leftrightarrow d(g h)=d(g)+g d(h) \Leftrightarrow$ $d \in \operatorname{Der}(G, M)$. 
Por outro lado, dados dois levantamentos $l_{1}(g)=\left(d_{1}(g), g\right)$ e $l_{2}(g)=$ $\left(d_{2}(g), g\right)$ temos que $l_{1}$ e $l_{2}$ sào $M$-conjugados $\Leftrightarrow l_{1}(g)=i^{\prime}(m) l_{2}(g) i^{\prime}(m)^{-1}$, para algum $m \in M$ e todo $g \in G \Leftrightarrow\left(d_{1}(g), g\right)=\left(m+d_{2}(g)-g m, g\right) \Leftrightarrow d_{2}(g)-d_{1}(g)=$ $g m-m=d_{m}(g) \Leftrightarrow d_{2}-d_{1} \in P(G, M)$.

Dai, facilmente, verifica-se que a aplicação

$$
\begin{aligned}
\frac{D c r(G, M)}{P(G, M)} & \rightarrow \mathcal{L} \\
d+P(G, M) & \rightarrow[l], \quad \text { onde } l(g)=(d(g), g),
\end{aligned}
$$

está bem definida e é uma bijeçào.

\section{Grupos de Dualidade}

Como foj dito no início deste capítulo, $R$ significará para nós o anel $\mathbf{Z}$ dos inteiros ou o corpo $\mathbb{Z}_{2}$.

Sabemos, por 1.4 (b), que na definişào de $H_{*}(G ; M)$ e $H^{*}(G ; M)$ (para $M$ um $R G$-módulo qualquer) podemos escolher uma resolução projetiva arbitrária $F=$ $\left(F_{i}\right)_{i \geq 0}$ de $R$ sobre $R G$.

Analogamente, se considerarmos do ponto de vista topológico (ver 1.9), entâo podemos computar $H_{0}(G ; M)$ e $H^{*}(G ; M)$ em termos de um complexo $K(G, 1)$ arbitrário $Y$.

Uma vez que temos esta liberdade de escolha é razoável tentar escolher $F$ (ou $Y$ ) como sendo o "menor" possível e isto nos leva a varias condiçòes de finitude sobre G.

Por exemplo, se interpretarmos "pequeno" em termos de comprimento de $F$ então somos levados à noção de dimensâo homológica e cohomológica. Ou ainda, se interpretamos pequeno por significar que cada $F_{\mathrm{i}}$ seja finitamente gerado então somos levados a assim chamada condiçâo $F P$.

Nesta seç̧ão introduzimos estas condiçôes de finitude sobre um grupo $G$, recordamos a definiçâo de grupos de dualidade e, em particular, de dualidade de Poincaré, e apresentamos (sem demonstraşào) alguns resultados relevantes desta teoria que serâo 
utilizados nos próximos capitulos. Finalizamos com alguns comentários sobre a conjectura de que todo grupo de dualidade de Poincaré sobre $\mathbf{Z}$ é o grupo fundamental de uma variedade asférica fechada.

Nossa referência básica para esta seç̧ão será as notas de R. Bieri [4]. Porém, quando for conveniente, referimos também a [5], [6] e [9]. Vale observar que K.S. Brown em [8, cap. VIII] apresenta um interessante tratamento deste assunto, mas somente sobre o anel $\mathbf{Z}$.

5.1 Definição: Dizemos que um grupo $G$ é de tipo $(F P)_{n}$ sobre $R, n \in \mathbb{N}$, se existe uma resolução projetiva de $R$ sobrc $R G, F \rightarrow R$, com $F_{i}$ finitamente gerado como $R G$-módulo, para todo $i \leq n$. Se os módulos $F_{i}$ sào finitamente geradospara todo $i$ então dizemos que $G$ é de tipo $(F P)_{\infty}$.

Observe que todo grupo $G$ é de tipo $(F P)_{0}$ sobre $R$ pois, por exemplo, a resoluçào bar (cf. 1.4 (c)) é uma resoluçào projetiva de $R$ sobre $R G$ em que $F_{0}$ é obviamente finitamente gerado. Para $n=1$, temos:

5.2 Proposição: [4, Prop. 2.1] $G$ é de tipo $(F P)_{1}$ sobre $R$ se, e somente se, $G$ é finitamente gerado.

Im resultado sobre grupos de tipo $(F P)_{n}$ que utilizaremos algumas vezes, nos casos simples $n=0,1$, é o seguinte:

5.3 Proposição: Sejam $G$ um grupo $\epsilon\left(M_{i}\right)_{i \in I}$ uma familia de $R G$ módulos. Se $G$ éde tipo $(F P)_{n}$, cntâo

$$
H^{k}\left(G ; \bigoplus_{i \in I} M_{i}\right) \simeq \bigoplus_{i \in I} H^{k}\left(G ; M_{i}\right), \quad \forall k \leq n
$$

Em particular, para qualquer grupo $G$ este isomorfismo $\dot{\epsilon}$ válido no nível $k=0 ; \epsilon$, se $G$ É finitamente gerado, tal isomorfismo sempre ocorre até o nível $k=1$.

5.4 Definição: Um grupo $G$ é denominado grupo de tipo $F P$ sobre $R$ se 
existe uma resoluçâo projetiva de $R$ sobre $R G$ de comprimento finito,

$$
0 \rightarrow F_{n} \rightarrow F_{n-1} \rightarrow \cdots \rightarrow F_{0} \rightarrow R \rightarrow 0
$$

com $F_{i}$ finitamente gerado para todo $i$.

5.5 Proposição: [9, Prop. 1.2] Seja G um grupo. As seguintes condiçōes sâo equivalentes:

(i) Se existe uma sequência crata

$$
0 \rightarrow K \rightarrow F_{n-1} \rightarrow \cdots \rightarrow F_{0} \rightarrow R \rightarrow 0
$$

com $F_{0}, \ldots, F_{n-1} R G$-projetivos, entào $h^{\prime}$ é também projetivo.

(ii) Existe uma resolusâo projetiva de $R$ sobre $R G$ de comprimento finito $n$

$$
0 \rightarrow F_{n} \rightarrow F_{n-1} \rightarrow \cdots \rightarrow F_{0} \rightarrow R \rightarrow 0
$$

(iii) $H^{k}(G ; M)=0$ para todo $R G$-módulo $M \epsilon$ todo $k>n$.

(iv) $H^{n+1}(G ; M)=0$ para todo $R G$-módulo $M$.

(v) Para todo epimorfismo $f: M \rightarrow M^{\prime}$, a aplicação induzida $f^{*}: H^{*}(G ; M) \rightarrow H^{*}\left(G ; M^{\prime}\right)$ é epimorfismo.

5.6 Definição: $A$ dimensão cohomológica de $G$ sobre $R$, denotada por $c d_{R} G, \dot{\epsilon}$ definida como sendo o menor intciro $n \geq 0$ que satisfaz uma das condiçóes equivalentes (i) a (v) da proposiçào anterior. Se nào existe tal inteiro consideramos $c d_{R} G=\infty$. Assim

$$
\begin{aligned}
c d_{R} G & =\inf \{n \mid R \text { admite uma resoluçào projetiva de comprimento } n\} \\
& =\inf \left\{n \mid H^{k}(G ;-)=0 \text { para } k>n\right\} \\
& =\sup \left\{n \mid H^{n}(G ; M) \neq 0 \text { para algum } R G \text {-módulo } M\right\} .
\end{aligned}
$$

Vale um resultado análogo à proposiçào anterior para homologia; porém, por simplicidade, definiremos a dimensâo homológica de $G$ sobre $R$, denotada por $h d_{R} G$, como

$$
h d_{R} G=\sup \left\{n ; H_{n}(G ; M) \neq 0 \text { para algum } R G \text {-módulo } M\right\} .
$$


5.7 Observaçōes: (a) Por conveniência, denotaremos $c d_{\mathbf{Z}_{2}} G$ e $h d_{\mathbf{Z}_{2}} G$ simplesmente por $c d G$ e $h d G$.

(b) É fácil ver que $G$ é de tipo $F P$ sobre $R$ se, e somente se, $G$ é de tjpo $(F P)_{\infty}$ sobre $R$ e $c d_{R} G<\infty$.

(c) R. Bieri em $[4$, cap. II] apresenta uma definiçào mais geral. Ele define dimensão plana (flat) e projetiva de um $\Lambda$-módulo $M$ ( $\Lambda$ anel qualquer com unidade), e então a dimensâo homológica (cohomológica) de um grupo $G$ é obtida da dimensào plana (projetiva) quando consideramos o $R G$-módulo particular $M=R$ com a $G$-a açào trivial.

5.8 Exemplo: Seja $G$ um grupo. Se existe uma variedade compacta Y de dimensâo $n$ que tem o mesmo tipo de homotopia de um complexo $h^{\prime}(G, 1)$ entâo. $c d_{R} G \leq n$. (cf. [8. VIII.8.1])

Apresentamos a seguir algumas das principais propriedades de dimensào homológica e cohomológica.

5.9 Proposição: [4, teor. 4.6] Scja G um grupo, entâo

(i) $h d_{R} G \leq c d_{R} G$.

(ii) St G' é cnumerável entâo $c d_{R} G \leq h d_{R} G+1$.

(iii) $S \epsilon G^{\prime} \dot{\epsilon}$ de tipo $(F P)_{\infty}$ sobre $R$. entâo $c d_{R} G=h d_{R} G$.

A próxima propriedade é consequencia imediata do lema de Shapiro.

5.10 Proposição: [4, Prop. 4.9]) Se St um subgrupo de G. cntâo

(i) $h d_{R} S \leq h d_{R} G$.

(ii) $c d_{R} S \leq c d_{R} G$.

5.11 Definição: Stjam $G$ um grupo $\in$ $R$ um ancl comutativo com unidade.

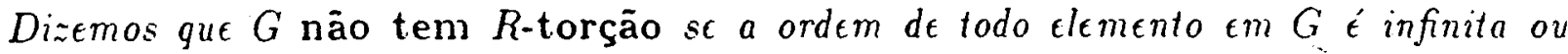
invertivel $\epsilon m R$ (isto $\epsilon$. a ordem é um inteiro $n>0$ tal que n.1 $\epsilon^{\prime}$ invertívét em $R$ onde 1 
é a unidade de $R$ ).

Observe que se $G$ não tem $\mathbb{Z}$-torção (isto é, se $G$ é livre de torçào) então obviamente $G$ nào tem $\mathbb{Z}_{2}$-torçào. A reciproca é falsa pois, por exemplo, $G=\mathbf{Z}_{3}$ não tem $\mathbf{Z}_{2}$-torçào mas não é $\mathbf{Z}$-livre de torçâo.

5.12 Proposição: [4, Props. 4.11 e 4.12]

(i) $S e h d_{R} G$ (ou $c d_{R} G$ ) é finita entâo $G$ nào tem $R$-torção.

(ii) $c d_{R} G=0$ se, e somente se, $G$ é um grupo finito sem $R$-torção.

(iii) $h d_{R} G=0$ se, $\epsilon$ somente se, $G \dot{\epsilon}$ um grupo localmente finito sem $R$ torçào.

O próximo resultado parte (i) é conhecido como Teorema de Serre.

5.13 Teorema: [4, teors. 5.11 e 5.13] Seja $S$ um subgrupo de indice finito em ùm grupo $G$. Se $G$ nâo tem $R$-torçào, então

(i) $c d_{R} S=c d_{R} G$.

(ii) $h d_{R} S=h d_{R} G$.

Grupos de Dualidade: Agora recordaremos o conceito de grupos de dualidade e suas propriedades mais relerantes. Brevemente falando, um grupo de dualidade de Poincaré $G$ (sobre $\mathbb{Z}$ ) é um grupo cuja homologia e cohomologia satisfazem relações de dualidade análogas àquelas válidas para variedades compactas. Estes grupos foram investigados, em vista de seus aspectos algébricos e topológicos, por Bieri em [3], e Johnson e Wall em [19] (1972). Posterjormente, Bieri e Eckmann em [6] (1973), definiram um tipo mais geral de dualidade (sobre $\mathbb{Z}$ ) que inclui o anterjor e leva a novas aplicaçōes em álgebra e topologia; e em [4, cap. 11I] (1976), Bierj extendeu este conceito por considerar grupos de dualidade sobre um anel qualquer comutativo com unidade $R$.

Aqui consideraremos somente os casos de interesse.

5.14 Definiçāo: Um grupo $G$ é denominado grupo de dualidade de dimensão $n$ sobre $R$, ou simplesmente um $D^{n}$-grupo sobre $R\left(\begin{array}{llll}R=\mathbb{Z} & \left.\text { ou } \mathbb{Z}_{2}\right)\end{array}\right.$ 
se existe um $R G$-módulo (à dircita) $C$, chamado módulo dualizante de $G$, tal que tenhamos isomorfismos naturais

$$
H^{k}(G ; M) \simeq H_{n-k}\left(G ; C \otimes_{R} M\right),
$$

para todo $k \in \mathbf{Z}$ e todo $R G$-módulo $M$, onde $C \otimes_{R} M$ é visto como $R G$-módulo com a $G$-ação diagonal.

$S \epsilon C \simeq R$ como $R G$-módulo dizemos que $G$ é um grupo de dualidade de Poincaré de dimensão n sobre $R$, ou simplesmente, um $P D^{n}$-grupo sobre $R$. Neste caso, se a ação de $G$ em $C$ é trivial (isto $\dot{e}, C$ é, a menos de isomorfismo, o G-módulo trivial $R$ dado em $1.2(b))$ dizcmos que $G$ é orientável, caso contrário $G$ é dito $G$ não orientável.

5.15 Observações: (a) Nos capítulos seguintes consideraremos sempre $R=\mathbb{Z}_{2}$, a menos que se especifique ao contrário. Assim, por conveniência, se $G$ é $D^{n}$. grupo sobre $\mathbb{Z}_{2}\left(P D^{n}\right.$-grupo sobre $\left.\mathbb{Z}_{2}\right)$ diremos, simplesmente, que $G$ é um $D^{n}$-grupo $\left(P D^{n}\right.$-grupo), sem falar no anel $\mathbb{Z}_{2}$.

Note que trabalhar com o anel $\mathbb{Z}_{2}$ tem a vantagem de que todo $P D^{n}$-grupo é orientável.

(b) Se $G$ é um grupo de dualidade de dimensâo $n$ sobre $R$, com mödulo dualizante $C$, então pode-se verificar que:

(i) $n=c d_{R} G$ e portanto $G$ nào tem $R$-torsào.

(ii) $C \simeq H^{n}(G ; R G$ i) como $R$ (i-módulos à direita, onde a estrutura de $R G$ módulo em $H^{n}(G ; R G)$ é induzida da estrutura natural de $R G$ como $R G$-módulo à direita, dada pela $G$-aşào multiplicaşào à direita.

Assim, a dimensào n e o módulo dualizante $C$ sâo determinados pelo grupo $G$.

5.16 Exemplo: Se $G=\Pi_{1}\left(X^{\prime}\right)$, onde $X$ é uma variedade fechada (compacta sem bordo), conexa, de dimensão n, asférica (isto é, que tem o mesmo tipo de homotopia de um complexo $K(G, 1)$ ) então $G$ é um $P D^{n}$-grupo sobre $\mathbb{Z}$ (ver [10, p. 135]). Em particular, $G=\Pi_{1}(X)$, onde $X$ é qualquer superfícje fechada diferente de $S^{2}$ e $\mathbb{R P}(2)$, 
é um $P D^{2}$-grupo sobre $\mathbf{Z}$, e $G=\mathbb{Z}^{n}=\Pi_{1}\left(S^{1} \times \ldots \times S^{1}\right)$ (n-vezes) é um $P D^{n}$-grupo sobre $\mathbf{Z}$.

Às vezes é conveniente trabalhar com a seguinte caracterização de grupos de dualidade.

5.17 Proposição: [4, teor. 9.2] Um grupo $G$ é um grupo de dualidade de dimensão n sobre $R$ se, e somente se, as seguintes condiçôes ocorre:

(i) $G$ é de tipo $F P$ sobre $R$.

(ii) $H^{k}(G ; R G)=0$ para $k \neq n$.

(iii) $H^{n}(G ; R G)=C$ é plano como $R$-módulo.

5.18 Corolário: Se $G$ é um grupo de dualidade sobre $R$ então $G$ é finitamente gerado.

Demonstração: Se $G$ é de dualidade então, pela proposição anterior $G$ é de tipo $F P$, e portanto de tipo $F P_{1}$ por 5.7 (b). Dai, o resultado segue de 5.2.

No teorema abaixo reunimos algumas propriedades de dualidade.

5.19 Teorema: [4. teors. $9.9,9.10$ e 9.11 ]

(i) Sejam $G$ um grupo scm R-torção e $S$ um subgrupo de indice finito em $G$. Então $G \dot{\epsilon}$ um $D^{n}$-grupo sobre $R$ st, $\epsilon$ somente st, $S \dot{\epsilon}, \epsilon$ temos, para os módulo dualizantes, o RS-isomorfismo

$$
H^{n}(S ; R S) \simeq R \in s_{S}^{G} H^{n}(G ; R G)
$$

(ii) Seja $N>G \stackrel{\pi}{\rightarrow} Q$ uma seguéncia trata curta de grupos. Se $N$ é um $D^{n}$-grupo sobre $R \in Q \dot{\epsilon}$ um $D^{q}$-grupo sobre $R$ então $G \dot{\epsilon}$ um $D^{n+q}$-grupo sobre $R$, $\epsilon$ os módulos duatizantes estào assim relacionados

$$
H^{r+q}(G ; R G) \simeq H^{q}(Q ; R Q) \circlearrowleft H^{n}\left(N^{\top} ; R N^{\prime}\right) \quad(\text { como } R G \text {-módulos })
$$

onde $G$ atua diagonalmente no produto tensorial (ver observação abaixo). 
(iii) Seja $N \longrightarrow G \stackrel{\pi}{\rightarrow} Q$ uma sequência exata $\operatorname{com} \operatorname{cd}_{R} Q<\infty$ e $N$ de tipo $(F P)_{\infty}$ sobre $R \quad\left(R=\mathbf{Z}\right.$ ou $\left.\mathbb{Z}_{2}\right)$. Se $G \dot{\epsilon}$ um grupo de dualidade sobre $R$ então também são $N \in Q$.

5.20 Observaçōes: (a) Podemos dar uma $G$-açâo à direita em $H^{n}(N ; R N)$ porque $N$ é normal em $G$. Tal ação pode ser assim descrita: Tomamos uma $R G$-resolução projetiva de $R, F \rightarrow R$, que por restriçâo é uma $R N$-resoluçâo projetiva de $R$ e definimos em $\operatorname{Hom}_{R N}(F, R N)$ a $G$-açâo à direita $(f . g)(x)=g^{-1} f(g x) g$, para todo $f \in \operatorname{Hom}_{R N}(F, R N), g \in G$ e $x \in F$. Esta ação induz a $G$-ação em $H^{n}(N ; R N)$ desejada.

A $G$-ação em $H^{q}(Q ; R Q)$ é induzida da $Q$-açâo.

(b) Quando consideramos $R$ um anel arbitrário qualquer (não necessariamente $\mathbb{Z}$ ou $\mathbb{Z}_{2}$ ), temos que exigir em 5.19 (iii) que $R$ seja um domínio de ideiais principais.

O resultado abaixo, embora simples, é muito importante para nós.

5.21 Lema: $S \epsilon G \dot{\epsilon}$ um $D^{n}$-grupo sobre $\mathbb{Z}$ com módulo dualizante $C$ então $G$ é um $D^{n}$-grupo (sobre $\mathbb{Z}_{2}$ ) com módulo dualizante $C^{\prime}=C \Theta_{\mathbf{Z}} \mathbb{Z}_{2}$. Em particular, st $G$ $\dot{\epsilon}$ um $P D^{n}$-grupo sobre $\mathbb{Z}$ entâo $G$ é um $P D^{n}$-grupo.

Demonstração: Segue de 1.7 e do fato que, para todo $\mathbb{Z}_{2} G$-módulo $M$, temos $C \otimes \mathbf{Z} M \simeq C \otimes_{\mathbf{Z}}\left(M \otimes_{\mathbf{Z}} \mathbb{Z}_{2}\right) \simeq C \otimes_{\mathbf{Z}}\left(\mathbb{Z}_{2} \otimes_{\mathbf{Z}} M\right) \simeq\left(C \otimes \mathbf{Z} \mathbb{Z}_{2}\right) \otimes_{\mathbf{Z}_{2}} M$.

Os grupos de dualidade de dimensào 0 e 1 sâo completamente determinados, como mostra o resultado seguinte.

5.22 Proposição: [4, Prop. 9.1i]

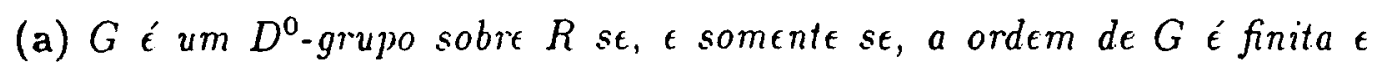
invertivel em $R$.

(b) $G$ é um $D^{1}$-grupo sobre $R$ se, $\epsilon$ somente se, $G$ é finitamente gerado $\epsilon$ 
$c_{R} G=1$

5.23 Exemplos: (a) $O$ grupo trivial $\{1\}$ é o único $D^{0}$-grupo sobre $\mathbf{Z}$.

(b) $\mathbf{Z}_{3}, \mathbf{Z}_{5}$ e mais geralmente todos os grupos finitos de ordem impar, sâo $D^{0}$-grupos (sobre $\mathbb{Z}_{2}$ ).

(c) $\mathbf{Z} * \mathbf{Z}$ é um $D^{1}$-grupo sobre $\mathbb{Z}$. Na realidade $G$ é um $D^{1}$-grupo sobre $\mathbf{Z}$ se, e somente se, $G$ é livre finitamente gerado; e $G \simeq \mathbb{Z}$ é o único $P D^{1}$-grupo sobre $\mathbf{Z}$ (ver [6, Prop. 5.1]).

(d) Segue do lema anterior e de 5.16, que se $G=\Pi_{1}(X)$ onde $X$ é um variedade asférica fechada de dimensão n entâo $G$ é um $P D^{n}$-grupo (sobre $Z_{2}$ ). Em particular, $\mathbb{Z}$ é um $P D^{1}$ e, por 5.19 (ii), $\mathbb{Z} \oplus \mathbb{Z}_{3}$ é um $D^{1}$-grupo.

Estes exemplos mostram claramente que a reciproca do lema anterior é falsa. De fato, o que vale é o seguinte resultado: Seja $G$ um grupo de tipo $F P$ sobre $\mathbb{Z}$. Então $G$ é um $D^{n}$-grupo sobre $\mathbb{Z}$ se, e somente se, $G$ é um $D^{n}$-grupo sobre todo corpo $\mathbb{Z}_{p}$ ( $p$ primo) e sobre o corpo de fraçôes $Q$ de $\mathbb{Z}$ (cf. [4, Prop. 9.13]).

O resultado seguinte será utilizado por nós no capítulo IV. O enunciaremos aqui somente no caso de interesse, a saber, quando $R=\mathbb{Z}_{2}$. Para a demonstraçào ver [5, prop. 5.6 e teor. 5.7$]$.

5.24 Proposição: Sejam $G$ um grupo com $c d G=n<\infty$ e $N$ um subgrupo normal $\epsilon m G$.

(a) Se $N$ é um $D^{n}$-grupo entâo $G$ também é, $\epsilon Q=G / N$ é finito.

(b) Suponhamos que $G$ seja finitamente gerado $\in N$ seja de tipo $F P$ sobre $\mathbb{Z}_{2}$, com $H^{k}\left(N ; \mathbb{Z}_{2} N\right)=0$ para $k \leq n-2$. Se $Q=G / N$ '́ infinito então $c d N^{\prime}=c d G-1$ $\epsilon \operatorname{dim} H^{1}\left(Q, \mathbb{Z}_{2} Q\right) \geq 1$ (ou equivalentemente, $Q$ tem mais $d \epsilon$ um $\epsilon$ d (ver cap II, 4.10)).

Finalizando esta seçào faremos um breve comentário sobre $P D^{n}$-grupos sobre $\mathbb{Z}$.

Conforme vimos no exemplo 5.16, se $G=\Pi_{1}(X)$, onde $X$ é um variedade 
asférica fechada de dimensào $n$, entào $G$ é um $P D^{n}$-grupo sobre $\mathbb{Z}$. $P D^{n}$-grupos sobre $\mathbf{Z}$ obtjdos desta forma são denominados geométricos.

Como todos os exemplos conhecidos de $P D^{n}$-grupos sobre $\mathbb{Z}$ são assim obtidos foi conjecturado que: Todo grupo de dualidade de Poincaré de dimensão $n$ sobre $\mathbf{Z}$ é geométrico.

$\mathrm{O}$ caso $n=1$ é óbvio, pois $G=\mathbb{Z}$ é o único $P D^{1}$-grupo sobre $\mathbb{Z}$, e $G=\Pi_{1}\left(S^{1}\right)$.

Em uma sequência de artigos $B$. Eckmann, H. Müller e P. Linnel [11] e [12], provaram que todos os $P D^{2}$-grupos sobre $\mathbb{Z}$ são geométricos, isto é, são grupos fundamentais infinitos de superfícies.

Para $n=3$, C.B. Thomas em [34] provou que, se $G$ é um $P D^{3}$-grupo sobre $\mathbb{Z}$ solúvel então $G$ é geométrico, de fato, $G$ é o grupo fundamental de uma 3-variedade asférica, orientável.

Não encontramos na literatura a prova da conjectura para o caso geral $n \geq 3$. Assim acreditamos que isto esteja ainda em aberto. 


\section{CAPÍTULO II}

\section{Um Invariante cohomológico para par grupos}

A partir deste capitulo trabalharemos sempre na categoria de $\mathbb{Z}_{2} G$-módulos.

Nós definiremos aqui um invariante cohomológico $E(G, \mathcal{S}, M)$ onde $G$ é um grupo, $\mathcal{S}=\left\{S_{i}\right\}_{i \in J}$ é uma familia de subgrupos com $\left[G: S_{i}\right]=\infty$, $\forall i \in I$, e $M$ é um $\mathbb{Z}_{2} G$-módulo.

Na primeira seç̧ão deste capitulo recordamos o conceito de (co)homologia relativa para pares $(G, \mathcal{S})$, introduzido por Bieri e Eckmann em [7], e alguns fatos que serão utilizados por nós.

Na seç̧ão 2 introduzimos o invariante $E(G, \mathcal{S}, M)$ e em seguida nos restringimos ao caso de interesse, ou seja. $\mathcal{S}=\{S\}$. Neste caso denotamos $E(G, \mathcal{S}, M) \mathrm{sim}$ plesmente por $E(G, S, M)$. Provamos que $E(G, S, M)$ é invariante no par $((G, S), M)$. Verificamos que $E(G, S, M)$ tem uma interpretação em termos de derivaçôes (no caso relatjvo) e derivaçoes principais, o que nos torna possivel computar, em alguns casos, tal invariante. Também comparamos os invariantes $E(G, T, M), E(G, S, M)$ e $E(G, S, N)$ quando $S$ e $T$ sào subgrupos de $G$. com $S \leq T$. e $M$ e $N$ sào $\mathbb{Z}_{2} G$-módulos. Ainda nesta seç̧ào (e até a seç̧âo 4), nos dedicamos ao invariante $E(G, S):=E\left(G, S, \mathbb{Z}_{2}(G / S)\right.$ ) (isto é, tomamos o módulo particular $M=\mathbb{Z}_{2}(G / S)$ ). Verificamos que $E(G, S)$ é invariante no par $(G, S)$ e concluímos a partir daí, que nào existe isomorfismos de pares para $\left(G=\langle a\rangle *\langle b\rangle \simeq \mathbb{Z} * \mathbb{Z} . \quad S=\left\langle a b a^{-1} b^{-1}\right\rangle\right)$ e $(G, K)=(\langle a\rangle *\langle b\rangle,\langle b\rangle)$.

Na seçâo 3 , após recordar o conceito de par de dualidade $\left(D^{n}\right.$-par) provamos o seguinte resultado: Dado um par $(G, S)$ com $[G: S]=\infty$, se $(G, S)$ é um $D^{n}$-par entâo $E(G, S)=1$. Assim nosso invariante dá uma condição necessária para que $(G, S)$ 
seja um $D^{n}$-par. Por exemplo, $(G, S)=(\langle a\rangle *\langle b\rangle,\langle b\rangle)$ não é um $D^{n}$-par pois $E(G, S)=\infty$. Finalizando, obtemos majoraçöes para $E(G, S)$ quando $G, S$ satisfazem outras propriedades de dualidade e exemplificamos.

Na secção 4, relacionamos o nosso invariante $E(G, S)$ com os invariantes ends $e(G)$ e $\epsilon(G, S)$. Recordamos incialmente a definiçào destes ends e suas propriedades majs relevantes, incluindo a relaçào com espaços topológicos. Se $G$ é infinito, tem-se que

$$
\epsilon(G)=1+\operatorname{dim} H^{1}(G, M)=E(G,\{1\})=1+\operatorname{dim} \frac{H^{1}(G,\{1\} ; M)}{P(G,\{1\}, M)}
$$

Uma vez que $E(G, S)=1+\operatorname{dim} \frac{H^{1}(G, S ; M)}{P(G, S, M)}$, nosso objetivo inicial nesta secçào era provar que

$$
\epsilon(G, S)=E(G, S)
$$

e assim obter uma fómula para $\epsilon(G, S)$ envolvendo cohomologia relativa. No entanto a igualdade (*) nem sempre é verdadeira, pois existem pares $(G, S)$ tais que $\epsilon(G, S) \dot{e}$ infinito enquanto que $E(G, S)$ é finito. Na realidade, provamos que $E(G, S) \leq \epsilon(G, S)$ e apresentamos alguns casos em que os invariantes coincidem, por exemplo, quando $S$ é normal em $G$. De fato apresentamos duas provas de que a igualdade é verdadeira no caso em que $S$ é normal em $G$.

Finalmente, na seçâo 5, apresentamos uma interpretaçào topológica para $E(G, S, M)$ (em particular para $E(G, S)$ ) em termos de pares Eilenberg-MacLane. Dai, o end $\epsilon(G, S)$ terá também tal interpretação topológica sempre que $\epsilon(G, S)=E(G, S)$.

\section{Cohomologia Relativa.}

Nesta seç̧âo nós recordamos a definição de cohomologia relativa e alguns fatos básicos, que serão de grande importancia para este trabalho.

A definiçào de cohomologia relativa para um grupo $G$ e uma família $\mathcal{S}=\left\{S_{i}\right\}_{i \in \mathcal{J}}$ de subgrupos de $G$, dada abaixo, foi introduzida por Bieri e Eckmann en 
[7] 1978. Tais autores consideraram os coeficientes na categoria de Z $G$-módulos, porém, tudo pode ser considerado, de modo análogo, na categoria de $\mathbb{Z}_{2} G$-módulos.

R. Ribes introduziu, anteriormente, em [24] 1969, a definiçào de cohomologia relativa $H^{k}(G, S ; M)$ para $G$ um grupo e $S$ um único subgrupo de $G$. Nós recordamos aqui este concejto, e provamos, uma vez que nào encontramos na literatura a justificativa deste fato, que a definiçâo devido a Ribes coincide (a menos de isomorfismo) com a de Bieri e Eckmann tomando $\mathcal{S}=\{S\}$. Assim, de fato, a definição de cohomologia introduzida por Bieri e Eckmann é uma generalizaçâo da apresentada por Ribes.

1.1: Um par grupo $(G, S)$ consiste de um grupo $G$ e uma familia não vazia $\mathcal{S}=\left\{S_{i}, i \in I\right\}$ de subgrupos de $G$ (nào necessariamente distintos).

Seja $\mathbb{Z}_{2}(G / \mathcal{S}) \circ \mathbb{Z}_{2}$-módulo livre gerado pelas classes $g S_{i}$, no qual $G$ atua por multiplicação à esquerda. Assim

$$
\mathbb{Z}_{2}(G / \mathcal{S})=\bigoplus_{i \in I} \mathbb{Z}_{2}\left(G / S_{i}\right)=\bigoplus_{i \in J}\left(\mathbb{Z}_{2} G \otimes_{S_{1}} \mathbb{Z}_{2}\right)
$$

Considere $\varepsilon: \mathbb{Z}_{2}(G / \mathcal{S}) \rightarrow \mathbb{Z}_{2}$ a aplicação aumentação usual, que aplica todo gerador $g S_{i}$ em 1 . Nós denotamos seu núcleo por $\Delta$, ou mais precisamente por $\Delta_{G / s}$.

1.2 Definição: $\operatorname{Sejam~}(G, \mathcal{S})$ um par grupo, $\mathcal{S}=\left\{S_{i}, i \in I\right\} \in M$ um $\mathbb{Z}_{2} G$-módulo. Os grupos de (co)homologia relativa para $(G, S)$ com coeficientes em $M$ sâo, para todo $k \in \mathbb{Z}$, assim definidos:

$$
\begin{aligned}
H^{k}(G, \mathcal{S} ; M) & =H^{k-1}(G ; \operatorname{Hom}(\Delta, M)) \\
H_{k}(G ; \mathcal{S} ; M) & =H_{k-1}(G ; \Delta \otimes M),
\end{aligned}
$$

onde o grupo Hom e o produto tensorial sâo vistos como $\mathbb{Z}_{2} G$-módulos com a açâo diagonal.

1.3 Observações: (a) A definição de homologia e cohomologia (absoluta) de grupos dada em l.1.3 pode ser vista como um caso particular da definição anterior se tomamos a família vazia $(S=\emptyset)$ e definimos, para todo $k \in \mathbb{Z}$,

$$
H_{k}(G, \emptyset ; M)=H_{k}(G ; M) \quad \text { e } \quad H^{k}(G, \emptyset ; M)=H^{k}(G ; M) .
$$


(b) Segue, claramente da definição, que para $k \leq 0$,

$$
H^{k}(G, \mathcal{S} ; M)=0 \text { e } H_{k}(G, \mathcal{S} ; M)=0 \text {. }
$$

(c) Se consideramos a definiçâo de cohomologia relativa na categoria de $\mathbf{Z}_{2} G$ e $\mathbf{Z} G$-módulos, pode-se provar um resultado análogo ao 1.1.7, isto é, dado um $\mathbb{Z}_{2} G$-módulo $M, H^{*}(G, \mathcal{S} ; M) \quad(H .(G, \mathcal{S} ; M))$ com $M$ visto como $\mathbb{Z}_{2} G$-módulo é isomorfo a $H^{*}(G, \mathcal{S} ; M) \quad(H .(G, \mathcal{S} ; M))$ para $M$ visto como $\mathbb{Z}_{2} G$-módulo. Para isto basta observar que, denotando por $\Delta^{\prime}=\operatorname{Ker}\left(\varepsilon^{\prime}: \mathbf{Z}(G / \mathcal{S}) \rightarrow \mathbb{Z}\right)$, temos os $\mathbb{Z}_{2} G$ isomorfismos $\operatorname{Hom}(\Delta, M) \simeq \operatorname{Hom} \mathbf{Z}\left(\Delta^{\prime}, M\right)$ e $\Delta \otimes M \simeq \Delta^{\prime} \otimes \mathbf{Z} M$, e daí, usar l.1.i e a definição de cohomologia relativa (sobre $\mathbb{Z}_{2}$ e $\mathbb{Z}$ ).

A nocào de cohomologia relativa para um par $(G, S)$ constituindo de um grupo $G$ e apenas um subgrupo $S$, dada por Ribes em [24], usa o conceito de funtor derivado. Precisamente, dado um $\mathbb{Z}_{2} G$-módulo $M$, considere o grupo abeliano

$$
\operatorname{Der}(G, S, M)=\left\{f \in \operatorname{Der}(G, M) \mid f_{\mid S}=0\right\} .
$$

onde $\operatorname{Der}(G, M)$ é como em 1.1.5.

É fácil ver que $\operatorname{Der}(G, S,-)$ é um funtor corariante, exato à esquerda, da categoria dos $\mathbb{Z}_{2} G$-módulos à esquerda na categoria dos grupos abelianos. Seja $0 \rightarrow M \rightarrow P_{0} \rightarrow P_{1} \rightarrow P_{2} \cdots \quad$ uma resoluçâo injetiva de $M$ (isto é, uma sequência exata de $\mathbb{Z}_{2} G$-módulos $P_{i}$. que sào injetivos para todo i). Temos entào

$$
0 \rightarrow \operatorname{D\epsilon r}(G, S, M) \rightarrow \operatorname{Der}\left(G, S, P_{0}\right) \rightarrow \operatorname{D\epsilon r}\left(G, S, P_{1}\right) \rightarrow \cdots
$$

Consideremos o complexo de cocadeias:

$$
\mathcal{C}^{*}: 0 \rightarrow D \epsilon r\left(G, S, P_{0}\right) \rightarrow \operatorname{Der}\left(G, S, P_{1}\right) \rightarrow \cdots .
$$

1.4 Definição: S€jam $G$ um grupo. $S$ um subgrupo $t M$ um $\mathbb{Z}_{2} G$-módulo. Entâo $H^{n+1}(G, S ; M)$ é o n-ésimo funtor derivado à dircita $d \epsilon$ $\operatorname{Der}(G, S, M)$, isto $\dot{\epsilon}$

$$
H^{n+1}(G, S: M):=H^{n}\left(\mathcal{C}^{*}\right)
$$


onde $\mathcal{C}^{*}$ é como acima.

1.5: Do fato que $\operatorname{Dcr}(G, S,-)$ é exato à esquerda, segue que

$$
H^{1}(G, S ; M)=\operatorname{Dcr}(G, S, M)
$$

1.6 Lema: Scjam $G$ um grupo $\epsilon \quad S$ um subgrupo de $G$. Então $H^{k}(G, S ; M) \simeq H^{k}(G,\{S\} ; M)$ para todo $k \in \mathbb{Z} \in$ todo $\mathbb{Z}_{2} G$-módulo $M$, ondc $H^{k}(G, S ; M)$ denota a cohomologia definida $\mathrm{\epsilon m} 1.4, \in H^{k}(G,\{S\} ; M)$ à dada $\mathrm{\epsilon m} 1.2$ tomando $\mathcal{S}=\{S\}$.

Demonstração: Por $[24, \operatorname{lema} 1.1), \operatorname{Dcr}(G, S,-) \simeq \operatorname{Hom}_{G}\left(\mathbb{Z}_{2}, \Gamma(-)\right)$ como funtor na categoria de $\mathbb{Z}_{2} G$-nódulos à esquerda, onde $\Gamma(M)=\frac{\operatorname{Hom}\left(\mathbb{Z}_{2} G, M\right)}{\operatorname{Hom} G\left(\mathbb{Z}_{2} G, M\right)}$ e daí

$$
H^{k}(G, S ; M) \simeq H^{k-1}(G ; \Gamma(M))
$$

Logo para provarmos o lema, é suficiente mostrar que $\Gamma(M)$ é $\mathbb{Z}_{2} G$-isomorfo a $\operatorname{Hom}(\Delta, M)$. Isto segue do seguinte diagrama comutativo com linhas exatas:

$$
\begin{aligned}
& 0 \rightarrow \operatorname{Hom}_{G}\left(\mathbb{Z}_{2} G, M\right) \stackrel{j}{\rightarrow} \operatorname{Hom}_{S}\left(\mathbb{Z}_{2} G, M\right) \stackrel{\rho}{\rightarrow} \quad \Gamma(M) \quad \rightarrow 0 \\
& \downarrow \psi \quad \text { (I) } \quad \downarrow \phi \quad \text { (II) } \quad \downarrow \varphi \\
& 0 \rightarrow \operatorname{Hom}\left(\mathbb{Z}_{2}, M\right) \quad \stackrel{\dot{\bullet}}{\rightarrow} \operatorname{Hom}\left(\mathbb{Z}_{2}(G / S), M\right) \stackrel{\stackrel{\bullet}{\rightarrow}}{H o m}(\Delta, M) \rightarrow 0
\end{aligned}
$$

As aplicaçòes horizontais $i^{*}$ e $\varepsilon^{*}$ sào as aplicaçôes induzidas da sequéncia exata $\quad 0 \rightarrow \Delta \stackrel{i}{\rightarrow} \mathbb{Z}_{2}(G / S) \stackrel{\iota}{\rightarrow} \mathbb{Z}_{2} \rightarrow 0 ; \quad j$ é a inclusào e $\rho$ é a projeção canônica. $\dot{E}$ fácil ver que estas aplicasòes sào $\mathbb{Z}_{2} G$-homomorfismos. Os $\mathbb{Z}_{2} G$-isomorfismos verticais $\psi$ e $\phi$ são dados por $\psi(f)(1)=f(1)$ e $\phi(f)(g S)=x f\left(g^{-1}\right)$. (De fato $\phi$ e $\psi^{\prime}$ são exatamente o $\mathbb{Z}_{2} G$-isomorfismo $v^{-1}$ dado em 1.3.12, tomando $M$ no lugar de $\Lambda$ e considerando, para obter $\xi: S=G)$.

A exatidào da primeira sequência é obria. Para a segunda sequência. tem-se que $\varepsilon^{*}$ é injetora e $\operatorname{lm} \varepsilon^{*}=$ Ker $i^{*}$ porque ofuntor $\operatorname{Hom}(-, M)$ é exato à esquerda. Agora, $i^{*}$ é sobrejetora porque $\mathbb{Z}_{2}$ é obviamente $\mathbb{Z}_{2}$-projetivo, e assim, $\varepsilon$ tem inversa á direita, ou equivalentemente (cf.[1S, 1.5.10]), $i$ tem inversa a esquerda. 
Também é fácil verificar que (l) é comutativo, e a aplicação $\varphi$ é definida de modo que (Il) seja comutativo, isto é,

$$
\begin{aligned}
\varphi: \Gamma(M) & \rightarrow \operatorname{Hom}(\Delta, M) \\
\rho(h) & \rightarrow\left(i^{*} \circ \phi\right)(h) .
\end{aligned}
$$

Daí pelo lema dos cinco ([18, 1.5.13]), $\varphi$ é um $\mathbf{Z}_{2} G$-isomorfismo como desejado.

Nós finalizamos esta seç̧ão com o seguinte resultado, que segue essencialmente do fato que a sequência exata curta de $\mathbb{Z}_{2} G$-módulos livres

$$
0 \rightarrow \Delta \rightarrow \mathbb{Z}_{2}(G / S) \rightarrow \mathbb{Z}_{2} \rightarrow 0
$$

permanece exata quando aplicamos o funtor $\operatorname{Hom}(-, M)$, lembrando ainda que

$$
H o m_{G}\left(\bigoplus_{i} \mathbb{Z}_{2}\left(G / S_{i}\right), M\right) \simeq \prod_{i} H o m_{G}\left(\mathbb{Z}_{2}\left(G / S_{i}\right), M\right)
$$

1.7 Proposição: [7, Prop.1.1] $S \epsilon \quad(G, \mathcal{S})$ é um par grupo, $\mathcal{S}=\left\{S_{i}, i \in I\right\}$, c $M \dot{\epsilon}$ um $\mathbb{Z}_{2} G$-módulo entôo temos a sequência exata longa:

$$
0 \rightarrow H^{0}(G ; M) \rightarrow \prod_{i} H^{0}\left(S_{i} ; M\right) \stackrel{\delta}{\rightarrow} H^{1}(G, \mathcal{S} ; M) \stackrel{J}{\rightarrow} H^{1}(G ; M) \stackrel{\text { res }}{\rightarrow} \prod_{i} H^{1}\left(S_{i} ; M\right) \rightarrow \cdots
$$

qué é natural no módulo $M$ e no par grupo $(G, \mathcal{S})$.

Uma consequência imediata da proposiçào anterior é que, se $S=\{S\}$ com $S=\{1\}$, entào $H^{k}(G ; M) \simeq H^{k}(G,\{1\} ; M)$, para todo $k \geq 2$ e todo $\mathbb{Z}_{2} G$-módulo $M$.

\section{2. $\mathrm{O}$ invariante $\mathrm{E}(\mathrm{G}, \mathcal{S}, \mathrm{M})$.}

Nós definiremos aqui um invariante que, como veremos no desenvolvimento do trabalho, generaliza de certo modo, os invariantes ends já existentes.

2.1 Definição: $\operatorname{Scja}(G, \mathcal{S})$ um par grupo, $\operatorname{com} \mathcal{S}=\left\{S_{i}, i \in I\right\} \epsilon$ $\left[G: S_{i}\right]=\infty$. Se $M$ é um $\mathbb{Z}_{2} G$-módulo definimos

$$
E(G, \mathcal{S}, M)=1+\operatorname{dim} \operatorname{Kier} r s_{S, M}^{G}
$$


onde ress, ${ }_{\mathcal{S}, M}^{G}: H^{1}(G ; M) \rightarrow \prod_{i} H^{1}\left(S_{i} ; M\right)$ é a aplicaşāo da sequéncia exata dada em 1.7.

Podemos observar, da exatidão da sequência 1.7, que Ker $\operatorname{res}_{s, M}^{G}=\operatorname{Im} J$ onde $J: H^{1}(G, S ; M) \rightarrow H^{1}(G ; M)$ e, se denotamos $\prod_{i} M^{s_{1}}$ por $M^{S}$, então

$$
\begin{aligned}
E(G, S, M) & =1+\operatorname{dim} \operatorname{Im} J \\
& =1+\operatorname{dim} \frac{H^{1}(G, \mathcal{S} ; M)}{\left(M^{S} / M^{G}\right)} .
\end{aligned}
$$

Seja $\mathcal{C}$ a seguinte categoria: um objeto de $\mathcal{C}$ é um par $\left(\left(G, S=\left\{S_{i}, i \in I\right\}\right) ; M\right)$ onde $(G, S)$ é um par grupo com $\left[G: S_{i}\right]=\infty$ e $M$ é um $Z_{2} G$-módulo. Uma aplicaçào em $\mathcal{C}$ de $\left(\left(G, \mathcal{S}=\left\{S_{i}, i \in I\right\}\right) ; M\right)$ em $\left(\left(L, \mathcal{H}=\left\{H_{j}, j \in J\right\}\right), N\right)$ é uma tripla $(\alpha, \pi, \phi)$ onde, $\alpha: G \rightarrow L \dot{e}$ um homomorfismo de grupos, $\pi: l \rightarrow J$ é uma aplicação tal que $a\left(S_{i}\right) \subset H_{\pi(i)}$, e $\phi: N \rightarrow M$ é um homomorfismo de grupos abelianos tal que $\phi(a(g) . n)=g . \phi(n)$ para $g \in G, n \in N$. Em outras palavras, $\phi$ é um homomorfismo de $\mathbb{Z}_{2} G$-módulos, onde $N$ é visto como $\mathbb{Z}_{2} G$-módulo por restriçào de escalares via $\alpha$, isto é, $g * n:=\alpha(g) \cdot n$.

Note que $(a, \pi, \phi)$ será um isomorfismo em $\mathcal{C}$ se, além disto, exigirmos que $\alpha$ e $\phi$ sejam isomorfismos de grupos, $\pi$ uma bijeção e $\alpha\left(S_{i}\right)=H_{\pi(i)}, \forall i \in I$. Podemos provar que $E(G, \mathcal{S}, M)$ é um invariante na categoria $\mathcal{C}$, isto é, se $((G, \mathcal{S}) ; M)$ e $((L, \mathcal{H}) ; N)$ sào isomorfos na categoria $\mathcal{C}$ entào $E(G, \mathcal{S}, M)=E(L, \mathcal{H}, N)$.

Em nosso trabalho estamos interessados somente no caso em que $\mathcal{S}=\{S\}$. Assim, provaremos aqui a invariança de $E(G, \mathcal{S}, M)$ para este caso (ver 2.3 abaixo). A demonstração, no caso genérico, está feita em detalhes em [1] e usa o fato que $H^{*}(G, \mathcal{S} ; M)$ é um funtor contravariante na categoria $\mathcal{C}$.

2.2 Observação: Por conveniência, no caso em que $S=\{S\}$ denotaremos o par grupo $(G,\{S\})$ por $(G, S)$ (que chamaremos muitas vezes de par de grupos ao invés de par grupo), e se $M$ é um $\mathbb{Z}_{2} G$-módulo, escreveremos $E(G, S, M)$ ao invés de $E(G,\{S\}, M)$. Observemos que neste caso a sequência exata dada em 1.7 tem (levando em conta 1.6) a forma mais simples: 
$0 \rightarrow H^{0}(G ; M) \rightarrow H^{0}(S ; M) \stackrel{6}{\rightarrow} H^{1}(G, S ; M) \stackrel{\jmath}{\rightarrow} H^{1}(G ; M) \stackrel{\text { res } \stackrel{G}{\rightarrow} M}{\rightarrow} H^{1}(S ; M) \rightarrow \cdots$

e se $[G: S]=\infty$,

$$
E(G, S, M)=1+\operatorname{dim} \operatorname{Ker} \operatorname{res}_{S, M}^{G},
$$

onde $r e s_{S, M}^{G}: H^{1}(G ; M) \rightarrow H^{1}(S ; M)$ é a aplicação restrição. (Quando nâo houver confusão denotaremos a aplicaşào $r \epsilon s_{S, M}^{G}$ simplesmente por $\left.r \epsilon s_{S}^{G}\right)$.

2.3 Proposição: $S \in((G, S) ; M)$ e $((L, H) ; N)$ sâo isomorfos na categoria $\mathcal{C}$ então

$$
E(G, S, M)=E(L, H, N) .
$$

Demonstração: Recordemos que $((G, S) ; M)$ e $((L, H) ; N)$ são isomorfos em $\mathcal{C}$ se existem um isomorfismo de grupos $a: G \rightarrow L$ tal que $\alpha(S)=H$, e um isomorfismo de grupos abelianos $\phi: M \rightarrow N$ satisfazendo $\phi(\alpha(g) \cdot n)=g \cdot \phi(n)$. Denotemos por $\alpha^{\prime}$ o isomorfismo restriçào $\alpha_{l}: S \rightarrow H$.

Consideremos o seguinte diagrama:

$$
\begin{array}{ccc}
H^{1}(G ; M) & \stackrel{r_{S} s_{S . M}^{G}}{\rightarrow} & H^{1}(S ; M) \\
(a, \phi)^{*} \downarrow & & \downarrow\left(a^{\prime}, \phi\right)^{*} \\
H^{1}(L ; N) & \stackrel{\operatorname{res}_{H, N}^{L}}{\rightarrow} & H^{1}\left(H ; N^{\prime}\right) .
\end{array}
$$

Podemos facilmente verificar que este diagrama é comutativo e consequentemente,

$$
(a, \phi)^{*}\left(\operatorname{Ker} r \epsilon s_{S, M}^{G}\right) \subset \operatorname{Ker} r e s_{H, N}^{L}
$$

pois, se $u \in \operatorname{Ker} r \epsilon s_{S, M}^{G}$, ou seja, $r \epsilon s_{S, M}^{G}(u)=0$, então $r \epsilon s_{H, N}^{L}\left((\alpha, \phi)^{*}(u)\right)=$ $\left(\alpha^{\prime}, \phi\right)^{*}\left(r e s_{S, M}^{G}(u)\right)=0$.

Portanto temos bem definido o homomorfismo de grupos

$$
(a, \phi)_{i}^{*}: \text { Ker } r s_{S, M}^{G} \rightarrow \operatorname{Ker} r \epsilon s_{H, N}^{L} .
$$

As aplicaçōes verticais $(\alpha, \phi)^{*}$ e $\left(\alpha^{\prime}, \phi\right)^{*}$ sâo isomorfismos uma vez que são induzidas de isomorfismos. 
Dai, $(a, \phi)_{i}^{\dagger}$ é obviamente injetora, e também sobrejetora pois, dado $v \in$ Ker $r \in s_{H, N}^{L} \subset H^{1}(L ; N)$, existe (do fato que $(\alpha, \phi)^{*}$ é sobrejetora) $u \in H^{1}(G ; M)$ tal que $(\alpha, \phi)^{*}(u)=v$. Mas tal $u \in \operatorname{Ker} \operatorname{res}_{S, N}^{G}$ pois $\left(\alpha^{\prime}, \phi\right)^{*}\left(r \epsilon s_{S, M}^{G}(u)\right)=\operatorname{res}_{H, N}^{L}\left((\alpha, \phi)^{*}(u)\right)=$ $r \epsilon s_{H, N}^{L}(v)=0$ e $\left(\alpha^{\prime}, \phi\right)^{*}$ é injetora.

Logo Ker $r e s_{S, M}^{G} \simeq$ Ker $r e s_{H, N}^{L}$, e portanto

$$
E(G, S, M)=1+\operatorname{dim} \operatorname{Ker} r \epsilon s_{S, M}^{G}=1+\operatorname{dim} \operatorname{Ker} r \epsilon s_{H, N}^{L}=E(L, H, N) .
$$

Nota: Conforme observação feita por Alejandro Adem, podemos sob certas hipóteses, espressar nosso invariante $E(G, S, M)$ em termos de característica de Euler parcial (mais 1), como mostra o lema abaixo.

2.4 Lema: Se os tres primeiros grupos de cohomologia da sequência exata 2.2 (1) tem dimensâo finita sobre $\mathbb{Z}_{2} \in[G: S]=\infty$, entâo

$$
E(G, S, M)=1+\operatorname{dim} H^{0}(G ; M)-\operatorname{dim} H^{0}(S ; M)+\operatorname{dim} H^{1}(G, S ; M) .
$$

Demonstração: Sabemos que, se $T: V_{1} \rightarrow V_{2}$ é um homomorfismo de espaços vetoriais (de dimensão finita), então $\operatorname{dim} \operatorname{Ker} T+\operatorname{dim} \operatorname{lm} T=\operatorname{dim} V_{1}$. Daí, usando este fato e a exatidão da sequencia 2.2 (1), obtemos:

$$
\begin{aligned}
\operatorname{dim} \operatorname{Ler} r \epsilon s_{S}^{G} & =\operatorname{dim} \operatorname{Im} J=\operatorname{dim} H^{1}(G, S ; M)-\operatorname{dim} \operatorname{Im} \delta \\
& =\operatorname{dim} H^{1}(G, S ; M)-\left(\operatorname{dim} H^{0}(S ; M)-\operatorname{dim} H^{0}(G ; M)\right)
\end{aligned}
$$

No resultado seguinte nós mostramos que o invariante $E(G, S, M)$ está relacionado com derivaşoes e derivaçoes principais. Isto é interessante pois torna possivel computar $E(G, S, M)$ em muitos casos (ver por exemplo 3.8 e 4.22 abaixo). O subgrupo das derivaçôes principais no caso relativo é dado por

$$
\begin{aligned}
P(G, S, M) & =\left\{d_{m} \in \operatorname{Der}(G, S, M), m \in M \mid d_{m}(g)=g m+m, \forall g \in G\right\} \\
& =\left\{d_{m} \in P(G, M) \mid d_{m \mid S}=0\right\} . \quad \text { (cf.I.1.5) }
\end{aligned}
$$

2.5 Lema: $S \epsilon \in \epsilon s_{S}^{G}: H^{1}(G ; M) \rightarrow H^{1}(S, M)$ é a aplicação restriçâo $€ n t a ̃ o$ 
Ker $r e s_{S}^{G} \simeq \frac{\operatorname{Der}(G, S, M)}{P(G, S, M)}$. Dai, se $[G: S]=\infty$, entào

$E(G, S, M)=1+\operatorname{dim} \frac{H^{1}(G, S ; M)}{P(G, S, M)}=1+\operatorname{dim} \frac{\operatorname{Der}(G, S, M)}{P(G, S, M)}$.

Demonstração: Uma vez que $H^{1}(G ; M)=\frac{\operatorname{Dcr}(G, M)}{P(G, M)}$ (por 1.1.6) e $H^{1}(G, S ; M)=\operatorname{Der}(G, S, M)$ (por 1.5), podemos ver a aplicação $J$ da sequência 2.2(1) como sendo a aplicação $\operatorname{Der}(G, S, M) \rightarrow \frac{\operatorname{Der}(G, M)}{P(G, M)}$, dada por $J(f)=f+P(G, M)$. Logo Ker $J=P(G, S, M)$ e daí, Ker $r s_{S}^{G}=\operatorname{Im} J \simeq \frac{\operatorname{Der}(G, S, M)}{P(G, S, M)}$.

Sabemos, por 1.4.3, que $\frac{\operatorname{Der}(G, M)}{P(G, M)}=H^{1}(G ; M)$ tem uma interpretação via levantamentos de uma extensão cindida de $G$ por $M$. Nós observamos que a seguinte generalizaçâo pode ser provada de modo análogo.

2.6 Proposição: Para todo $\mathbb{Z}_{2} G$-módulo $M$, as classes de $M$-conjugação [l] de levantamentos $l: G \rightarrow M \rtimes G$ satisfazendo $l_{S}=\left(0, I d_{S}\right)$, da extensão cindida

$$
0 \rightarrow M \rightarrow M \rtimes G \rightarrow G \rightarrow 1
$$

estâo em correspondência biunivoca com os elementos de $\frac{\operatorname{Der}(G, S, M)}{P(G, S, M)}$.

Demonstração: Seja $\mathcal{L}^{\prime}$ o conjunto das classes de $M$-conjugasâa $[l]$ tal que $l_{S}=\left(0, i d_{S}\right)$.

Claramente, um levantamento $l$ satisfaz $l_{\mid S}=\left(0, i d_{S}\right)$ se, e somente se, a derivação correspondente $d$ (ver demonstração I.4.3) satisfaz $d_{\mid S}=0$, isto é, $d \in \operatorname{Der}(G, S, M)$. Assim, temos bem definida a aplicaçâo

$$
\begin{aligned}
\frac{D \epsilon r(G, S, M)}{P(G, S, M)} & \rightarrow \mathcal{L} \\
d+P(G, S, M) & \rightarrow l \text { tal que } l(g)=(d(g), g) .
\end{aligned}
$$

e facilmente verifica-se que tal aplicaçâo é bijetora.

A seguir apresentamos algumas propriedades interessantes do invariante $E$. 


\subsection{Proposição:}

(i) Sejam $S, T$ subgrupos de $G$ satisfazendo $S \leq T \leq G$. Sc $[G: T]=\infty$

entâo

$$
E(G, T, M) \leq E(G, S, M)
$$

para todo $\mathbf{Z}_{2} G$-módulo $M$.

(ii) Sejam $N, M \mathbb{Z}_{2} G$-módulos. Se existe um $\mathbb{Z}_{2} G$-homomorfismo $\phi: N \rightarrow M$ tal que o homomorfismo induzido $\phi^{*}: H^{1}(G ; N) \rightarrow H^{1}(G ; M) \dot{\epsilon}$ monomorfismo, entào

$$
E(G, S, N) \leq E(G, S, M)
$$

para todo subgrupo $S$ de $G$ satisfazendo $[G: S]=\infty$.

Demonstração: (i) Se $S \leq T \leq G$ entào $r \epsilon s_{S}^{G}=r \epsilon s_{S}^{T} \circ r \epsilon s_{T}^{G}$, onde

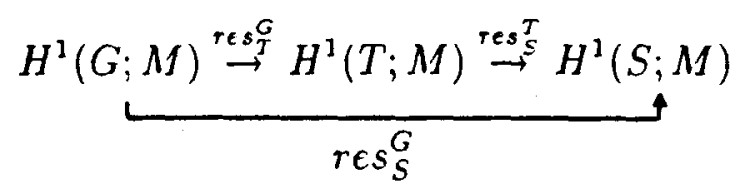

Logo, Ker $r e s_{T}^{G} \subset$ Ker $r \epsilon s_{S}^{G}$. Daí

$$
E(G, T, M)=1+\operatorname{dim} \operatorname{Ker} r \epsilon s_{T}^{G} \leq 1+\operatorname{dim} \operatorname{Ker} r \epsilon s_{S}^{G}=E(G, S, M) .
$$

(ii) Consideremos o diagrama comutativo

$$
\begin{array}{ccc}
H^{1}\left(G ; N^{\prime}\right) & \stackrel{\phi^{\bullet}}{\rightarrow} & H^{3}(G ; M) \\
\downarrow r \epsilon s_{S, N}^{G} & & \downarrow r \epsilon s_{S, M}^{G} \\
H^{1}\left(S ; N^{*}\right) \stackrel{\left(i d_{\underline{S}, \phi}\right)^{\bullet}}{\rightarrow} & H^{1}(S ; M)
\end{array}
$$

Da comutatividade do diagrama, segue que $\phi^{*}\left(\right.$ her $\left.r \varepsilon_{S, N}^{G}\right) \quad \subset$ Ker $r \epsilon s_{S, M}^{G}$. Como $\phi^{*}$ é monomorfismo temos que $\operatorname{dim} \operatorname{Ker} r \epsilon s_{S, N}^{G}=\operatorname{dim} \phi *\left(\operatorname{Ker} r \epsilon s_{S, N^{N}}^{G}\right)$. Daí,

$$
E(G, S, N)=1+\operatorname{dim} \text { Ker } r \epsilon s_{S, N}^{G} \leq 1+\operatorname{dim} \operatorname{ker} r \epsilon s_{S, M}^{G}=E(G, S, M)
$$

2.8 Proposição: Sejam $S, T$ subgrupos de $G$ satisfazendo $S \leq T \leq G$ e $M$ um $\mathbb{Z}_{2} G$-módulo. Se $[T: S]=\infty \quad \epsilon$

$$
\varphi^{*}: H^{1}\left(G ; \operatorname{Ind} d_{T}^{G}\left(\operatorname{In} d_{S}^{T} M\right)\right) \rightarrow H^{1}\left(G ; \operatorname{Coind}_{T}^{G}\left(\operatorname{In} d_{S}^{T} M\right)\right)
$$


é um monomorfismo, onde $\varphi^{*} \quad \dot{c}$ a aplicaçào induzida do mergulho natural $\varphi: \operatorname{lnd} d_{T}^{G}\left(\operatorname{Ind} d_{S}^{T} M\right) \rightarrow C$ oind $d_{T}^{G}\left(\operatorname{Ind} d_{S}^{T} M\right)$ dado $\mathrm{cm} I .3 .6$ (i), então

$$
E\left(G, S, \operatorname{In} d_{S}^{G} M\right) \leq E\left(T, S, \operatorname{Ind} d_{S}^{T} M\right)
$$

Demonstração: Consideremos as seguintes aplicaçòes

$$
\operatorname{Ind} d_{S}^{G} M \stackrel{\Downarrow}{\rightarrow} \operatorname{Ind} d_{T}^{G} \operatorname{In} d_{S}^{T} M \stackrel{\varphi}{\rightarrow} \operatorname{Coind} d_{T}^{G} \operatorname{Ind} d_{S}^{T} M \stackrel{\pi}{\rightarrow} \ln d_{S}^{T} M
$$

onde nos geradores $\psi$ é dada por $\psi(g \otimes m)=g \otimes 1 \otimes m$ e é estendida por linearidade, $\varphi$ é o $\mathbb{Z}_{2} G$-monomorfismo dado em I.3.6 (i) e $\pi$ é o $\mathbf{Z}_{2} T$-epimorfismo como em 1.3.4, isto $\dot{e}, \pi(\varphi(g \otimes 1 \otimes m))=\varphi(g \otimes 1 \otimes m)(1)$.

Claramente $\psi$ é um $\mathbb{Z}_{2} G$-isomorfismo, e portanto $\phi=\pi \circ \varphi \circ \psi$ é um $\mathbb{Z}_{2} T$-homomorfismo.

O par de aplicaçōes $(T \stackrel{a}{\hookrightarrow} G, \phi)$ induz o homomorfismo

$$
(\alpha, \phi)^{*}: H^{1}\left(G ; \operatorname{In} d_{S}^{G} M\right) \rightarrow H^{1}\left(T ; \operatorname{Ind} d_{S}^{T} M\right)
$$

Como $(\alpha, \phi)^{*}=(a, \pi)^{*} \circ \varphi^{*} \circ \psi^{*}$, com

$$
H^{1}\left(G ; \operatorname{Ind} d_{S}^{G} M\right) \stackrel{\psi^{*}}{\rightarrow} H^{1}\left(G ; \operatorname{Ind} d_{T}^{G} \operatorname{Ind} d_{S}^{T} M\right) \stackrel{\varphi^{*}}{\rightarrow} H^{1}\left(G ; \operatorname{Coind} T_{T}^{G} \operatorname{Ind} d_{S}^{T} M\right) \stackrel{(a, \pi)^{*}}{\rightarrow} H^{1}\left(T ; \operatorname{Ind} d_{S}^{T} M\right)
$$

onde $(a, \pi)^{*}$ é o isomorfismo do lema de Shapiro (1.3.14), segue da hipótese da proposição e do fato que $\psi *$ é obviamente isomorfismo, que $(a, \phi)^{*}$ é um monomorfismo.

Consideremos o seguinte diagrama comutativo:

$$
\begin{aligned}
& H^{1}\left(G ; \operatorname{In} d_{S}^{G} M\right) \stackrel{r \in s_{S}^{G}}{\rightarrow} H^{1}\left(S ; \operatorname{In} d_{S}^{G} M\right) \\
& \qquad(a, \phi)^{*} \quad \quad \downarrow \phi^{*}=(i d: S \rightarrow S, \phi)^{*} \\
& H^{1}\left(T ; \operatorname{In} d_{S}^{T} M\right) \stackrel{r \in s_{S}^{T}}{\rightarrow} H^{1}\left(S ; \operatorname{In} d_{S}^{T} M\right)
\end{aligned}
$$

Se $[f] \in$ lier $r \epsilon s_{S}^{G}$ segue, da comutatividade do diagrama, que $(a, \phi)=([f]) \in \operatorname{ker} r \epsilon s_{S}^{T}$. Portanto está bem definida a aplicaçâo

$$
(a, \phi)_{1}^{*}: \text { Ker } r \epsilon s_{S}^{G} \rightarrow \text { Ker } r \epsilon s_{S}^{T} .
$$


Dai, como $(\alpha, \phi)^{\bullet}$ é monomorfismo, temos que

$$
\begin{aligned}
E\left(G, S, \ln d_{S}^{G} M\right) & =1+\operatorname{dim} \text { Ker } \operatorname{res}_{S}^{G} \\
& \leq 1+\operatorname{dim} \operatorname{Ker} r e s_{S}^{T}=E\left(T, S, \operatorname{Ind}_{S}^{T} M\right) .
\end{aligned}
$$

2.9 Corolário: Sejam $S, T$ subgrupos de $G$ satisfazendo $S \leq T \leq G$, e $M$ um $\mathbb{Z}_{2} G \cdot$ módulo. Se $[G: S]=\infty \in[G: T]<\infty$ então

$$
E\left(G, S, \operatorname{In} d_{S}^{G} M\right) \leq E\left(T, S, \operatorname{In} d_{S}^{T} M\right) .
$$

Demonstração: Se $[G: T]=\infty$ entâo temos por I.3.6(ii), que $\operatorname{Ind} d_{T}^{G} \stackrel{\mathscr{}}{\simeq}$ $\operatorname{Coind}_{T}^{G} N$, para todo $\mathbb{Z}_{2} T$-módulo $N$, em particular, para $N=\operatorname{Ind} T$ S. Logo, a aplicaçâo induzida $\varphi^{*}: H^{1}\left(G ; \ln d_{T}^{G} \ln d_{S}^{T} M\right) \rightarrow H^{1}\left(G ; C o i n d_{T}^{G} \operatorname{In} d_{S}^{T} M\right)$ é um $\mathbf{Z}_{2} G$-isomorfismo e portanto o resultado segue da proposição anterior.

\section{$O$ invariante $E(G, S)$ :}

Seja $G$ é um grupo e $S$ um subgrupo de $G$ com $[G: S]=\infty$. Nós fixaremos, a partir de agora, o $\mathbb{Z}_{2} G$-módulo particular $M=\mathbb{Z}_{2}(G / S) \simeq \operatorname{Ind} d_{S}^{G} \mathbb{Z}_{2}$ (ver 1.3.13(a)). Por simplicidade, $E\left(G, S, \mathbb{Z}_{2}(G / S)\right)$ será denotado por $E(G, S)$.

2.10 Lema: $E(G, S)$ é um invariante algébrico para pares de grupos $(G, S)$ $\operatorname{com}[G: S]=\infty$, isto $e_{\text {, se }} a:(G, S) \rightarrow\left(L, K^{\prime}\right)$ é um isomorfismo de pares de grupos, então $E(G, S)=E\left(L, K^{\prime}\right)$.

Demonstração: Seja a $:(G, S) \rightarrow\left(L, K^{\prime}\right)$ um isomorfismo de pares de grupos, isto é, $a: G \rightarrow L$ é um isomorfismo de grupos com $a(S)=K$. Por 2.3 é suficiente provar que existe $\phi: \mathbb{Z}_{2}\left(L / l^{\prime}\right) \rightarrow \mathbb{Z}_{2}(G / S)$, isomorfismo de grupos abelianos, tal que $\phi(a(g) \cdot u)=g . \phi(u)$, para $g \in G$ e $u \in \mathbb{Z}_{2}(L / K)$ em outras palavras, $\left((G, S) ; \mathbb{Z}_{2}(G / S)\right)$ e $\left(\left(L, K^{\prime}\right) ; \mathbb{Z}_{2}\left(L / K^{\prime}\right)\right)$ sào isomorfos na categoria $\mathcal{C}$.

Considere $\phi: \mathbb{Z}_{2}\left(L / K^{\prime}\right) \rightarrow \mathbb{Z}_{2}(G / S)$ definida nos geradores por $\phi\left(x K^{\prime}\right)=a^{-1}(x) S$ para $x \in L$, e estendida por linearidade. Claramente $\phi \dot{e}$ um isomorfismo de grupos. Além disto, $\phi\left(\alpha(g) . x K^{\prime}\right)=\phi\left((\alpha(g) x) K^{\prime}\right)=\alpha^{-1}(\alpha(g) x) S=\left(g \alpha^{-1}(x)\right) S=$ $g . \phi\left(x K^{\prime}\right)$. Portanto $\phi$ está nas condiçôes desejada. 
2.11 Observação: A existència de isomorfismos separados $G \rightarrow L$ e $S \rightarrow K$ não implica que $E(G, S)=E(L, K)$.

De fato, seja $G=\langle a\rangle *\langle b\rangle \simeq \mathbb{Z} * \mathbb{Z}$ e consideremos os seguintes subgrupos de $G$ :

$$
S=\left\langle a b a^{-1} b^{-1}\right\rangle \text { e } K=\langle b\rangle
$$

Temos obviamente que $S \simeq K \simeq \mathbf{Z}$. No entanto, veremos na seç̧ão seguinte que, $E(G, S)=1$ enquanto que $E\left(G, K^{\prime}\right)=\infty$ (ver 3.7 e 3.8 , respectivamente).

Logo, como uma aplicação do nosso invariante, podemos concluir que não existe isomorfismos de pares de grupos $\left(<a>*<b>,\left\langle a b a^{-1} b^{-1}\right\rangle\right) \rightarrow$ $(<a\rangle *\langle b\rangle,\langle b\rangle)$.

Concluindo esta seç̧ão nós reescreveremos, para facilitar futuras referências, o corolário 2.9 em termos do invariante $E(G, S)$.

2.12 Corolário: Sejam $S \in T$ subgrupos de $G$ satisfazendo $S \leq T \leq G$. Se $[G: S]=\infty$ e $[G: T]<\infty$, entào

$$
E(G, S) \leq E(T, S)
$$

Demonstração: Basta considerar, em 2.9, $M=\mathbb{Z}_{2}$.

\section{3. $\mathrm{E}(\mathrm{G}, \mathrm{S})$ e Dualidade.}

Nesta seç̧ão apresentamos alguns resultados para o invariante $E(G, S)$ quando $G, S$ ou $(G, S)$ satisfazem certas condiçoes de dualidade. Dentre eles destacamos o seguinte: "Se $(G, S)$ é um par de dualidade de dimensâon $\left(D^{n}\right.$-par) entâo $E(G, S)=1$ ". Assim, nosso invariante $E(G, S)$ dá uma condição necessária para que $(G, S)$ seja um $D^{n}$-par.

Este resultado é uma "generalização" do resultado já existente para um grupo $G$. A saber, "se $G$ é um $D^{n}$-grupo $(n>1)$ entào $e(G)=1$ " (ver 4.11 , na seç̧ão seguinte). 
0 conceito de par de dualidade para um par grupo $(G, \mathcal{S}), \mathcal{S}=\left\{S_{i}\right\}_{i \in l}$ foi introduzido por Bieri e Eckmann em [7, p. 299] para o anel $R=\mathbf{Z}$. No entanto, tudo pode ser feito de modo análogo para $R=\mathbf{Z}_{2}$. É neste caso que estamos interessados e na família $\mathcal{S}=\{S\}$. Para facilitar ao leitor, nós recordamos aqui este conceito e suas propriedades mais relevantes. Para major detalhes ver referência acima.

3.1 Definição: Um par grupo $(G, \mathcal{S})$ é chamado um par de dualidade de dimensão $n$ (sobre $\mathbf{Z}_{2}$ ), ou simplesmente um $D^{n}$-par, se existe um $\mathbf{Z}_{2} G$-módulo $C$ $e$ isomorfismos naturais:

$$
\begin{aligned}
& H^{k}(G ; M) \simeq H_{n-k}(G, \mathcal{S} ; C \otimes M) \quad \epsilon \\
& H^{k}(G, \mathcal{S} ; M) \simeq H_{n-k}(G ; C \otimes M)
\end{aligned}
$$

para todo $\mathbb{Z}_{2} G$-módulo $M \in$ todo $k \in \mathbb{Z}$.

$C$ é chamado o módulo dualizante de $(G, \mathcal{S})$. Se $C \simeq \mathbb{Z}_{2}$ como um grupo abeliano, o par de dualidade $(G, \mathcal{S})$ é chamado um par de dualidade de Poincaré, ou simplesmente um $P D^{n}$-par (neste caso os isomorfismos (1) e (2) são equivalentes).

3.2 Observações:(a) A definição de $D^{n}$-grupos (sobre $\mathbf{Z}_{2}$ ) dado em I.5.14, pode ser vista como un caso particular do conceito de $D^{n}$-pares se consideramos o par grupo $(G, \mathcal{S})$ com $\mathcal{S}=\emptyset \quad$ (familia vazja) e tomamos, como em 1.3(a),

$$
H^{k}(G, \emptyset ; M)=H^{k}(G ; M) \text { e } H_{k}(G, \emptyset ; M)=H_{k}(G ; M) .
$$

(b) Se $(G, \mathcal{S})$ é um $D^{n}$-par com módulo dualizante $C$ entâo

$$
H^{n}\left(G, \mathcal{S} ; \mathbb{Z}_{2} G\right) \simeq C \quad \text { (como } \mathbb{Z}_{2} G \text {-módulos) }
$$

Dai, $C$ é determinado pelo par $(G, \mathcal{S})$ e, uma vez que $H^{k}(G, \mathcal{S} ; M)=0$ para todo $M$ e $k>n$, e $H^{n}\left(G, \mathcal{S} ; \mathbb{Z}_{2} G\right)=C \neq 0$, temos que o inteiro $n$ é também determinado por $(G, \mathcal{S})$ (compare com 1.5.15 (b)).

(c) Como em I.5.21 pode-se provar, levando em consideração 1.3(c), que se $(G, \mathcal{S})$ é um $D^{n}$-par sobre $\mathbb{Z}$ entào $(G, \mathcal{S})$ é um $D^{n}$-par sobre $\mathbb{Z}_{2}$. 
3.3 Proposição: $\left[7\right.$, teor. 4.2] Se $(G, \mathcal{S}), \quad \mathcal{S}=\left\{S_{i}, i \in I\right\} \neq \emptyset$, é um $D^{n}$-par com módulo dualizante $C$, entâo

(i) $G$ é um $D^{\text {n-1 }}$-grupo com módulo dualizante $\Delta \otimes C$ (com a G-açào diagonal).

(ii) Cada $S_{i}$ é um $D^{n-1}$-grupo com módulo dualizanté $C$ (considerado como $S_{i}$-módulo por restriçâo).

(iii) $\mathcal{S}$ é uma familia finita de subgrupos.

Antes de estabelecer o próximo resultado, que nos dá exemplos de $P D^{n}$-pares, recordamos aqui quando um par grupo $(G, \mathcal{S})$ é realizado topologicamente por um par Eilenberg-MacLane.

3.4: Seja $(G, \mathcal{S})$ um par grupo, onde $\mathcal{S}=\left\{S_{i}, i \in I\right\}$. Dizemos que $(G, \mathcal{S})$ é realizado topologicamente por um par Eilenberg-MacLane $(X, Y)=K(G, \mathcal{S}, 1)$ se:

(i) $X$ é um complexo celular $h^{\prime}(G, 1)$

(ii) $Y$ é um subcomplexo cujas componentes $Y_{i}, i \in I$, são complexos $K\left(S_{i}, 1\right)$; o que significa que a aplicação $\pi_{1}\left(Y_{i}\right) \rightarrow \pi_{1}(X)$, induzida pela inclusão $Y_{i} \subset X$, é injetiva e aplica $\pi_{1}\left(Y_{i}\right)$ sobre $S_{i} \subset G$ (depois de uma conveniente escolha de caminhos conectando pontos base).

3.5 Teorema: [7, teor. 6.3$] S \epsilon(G, \mathcal{S})$ é realizado topologicanente por um par Eilemberg-MacLane $(X, Y)$ onde $X$ é uma variedade de dimensão n, com bordo, compacta, orientável $\epsilon \quad Y=\partial X \quad$ (nâo necessariamente conexo), entâo $(G, \mathcal{S})$ é um $P D^{n}$-par.

Nota: De fato, em [7] conclui-se que, nas hipóteses acima, $(G, S)$ é um $P D^{n}$-par sobre $\mathbb{Z}$ mas, por 3.2(c), tem-se que $(G, \mathcal{S})$ é um $P D^{n}$-par (sobre $\mathbb{Z}_{2}$ ).

Agora estamos em condiçôes de estabelecer e exemplificar o nosso resultado.

3.6 Proposição: Sejam $G$ um grupo $\epsilon S$ um subgrupo $d \epsilon G$ com $[G: S]=\infty$. Se $(G, S) \dot{\epsilon}$ um $D^{n}$-par, então $E(G, S)=1$. Ou equivalentemente; st 
$E(G, S) \neq 1$ então $(G, S)$ nào é um $D^{n}$-par.

Demonstração: Observemos, incialmente, que se $(G, S)$ é um $D^{1}$-par então, por 3.3, $G$ e $S$ sâo $D^{0}$-grupos (sobre $\mathbb{Z}_{2}$ ). Portanto são grupos finitos (por 1.5.22). Dai $[G: S\}<\infty$. Logo, nas hipóteses acima, temos necessariamente $n>1$.

Considere a sequencia exata 2.2(1) $\operatorname{com} M=\mathbf{Z}_{2}(G / S)$,

$$
\begin{aligned}
0 \rightarrow H^{0}\left(G ; \mathbf{Z}_{2}(G / S)\right) \rightarrow & H^{0}\left(S ; \mathbb{Z}_{2}(G / S)\right) \stackrel{\delta}{\rightarrow} H^{1}\left(\left(G, S ; \mathbf{Z}_{2}(G / S)\right) \stackrel{J}{\rightarrow}\right. \\
& \rightarrow H^{1}\left(G ; \mathbb{Z}_{2}(G / S)\right) \stackrel{\text { resG }}{\rightarrow} H^{1}\left(S ; \mathbb{Z}_{2}(G / S)\right) \rightarrow \cdots
\end{aligned}
$$

Seja $C$ o módulo dualizante de $(G, S)$. Entâo

$$
\begin{aligned}
H^{1}\left(G, S ; \mathbb{Z}_{2}(G / S)\right) & \simeq H_{n-1}\left(G ; C \otimes \mathbb{Z}_{2}(G / S)\right) \\
& \simeq H_{n-1}\left(G ; C \otimes I_{n}^{G} \mathbb{Z}_{2}\right) \\
& \simeq H_{n-1}\left(G ; \operatorname{Ind} d_{S}^{G}\left(C \otimes \mathbb{Z}_{2}\right)\right) \\
& \simeq H_{n-1}\left(S ; C \otimes \mathbb{Z}_{2}\right) \\
& \simeq H^{0}\left(S ; \mathbb{Z}_{2}\right) \\
& \simeq \mathbb{Z}_{2}
\end{aligned}
$$

(por Lema de Shapiro: 1.3.14)

Agora, observe que $H^{0}\left(G ; \mathbb{Z}_{2}(G / S)\right)=\left(\operatorname{In} d_{S}^{G} \mathbb{Z}_{2}\right)^{G}=0$ uma vez que $[G: S]=\propto($ ver 1.3 .11$)$, e $H^{0}\left(S ; \mathbb{Z}_{2}(G / S)\right)=\mathbb{Z}_{2}(G / S)^{S}$ contém $\{0,1 S\} \simeq \mathbb{Z}_{2}$. Daítemos $\mathbb{Z}_{2} \subset \mathbb{Z}_{2}(G / S)^{S} \stackrel{\delta}{\succ} \mathbb{Z}_{2}$ (con $\delta$ monomorfismo): o que nos dá $\mathbb{Z}_{2}(G / S)^{S} \simeq \mathbb{Z}_{2}$. Assim, $\operatorname{Ker} r \epsilon s_{S}^{G}=\operatorname{Im} J \simeq \frac{H^{1}\left(G, S ; \mathbb{Z}_{2}(G / S)\right)}{\mathbb{Z}_{2}(G / S)^{S}}=0$ e portanto $E(G, S)=1$.

3.7 Exemplo: Se $G=\langle a\rangle *\langle b\rangle \simeq \mathbb{Z} * \mathbb{Z}$ e $S=\left\langle a b a^{-1} b^{-1}\right\rangle \simeq \mathbb{Z}$ então $E(G, S)=1$. De fato, seja $X$ um toro dos quais um disco aberto $D^{2}$ foi removido como mostra a figura abaixo:

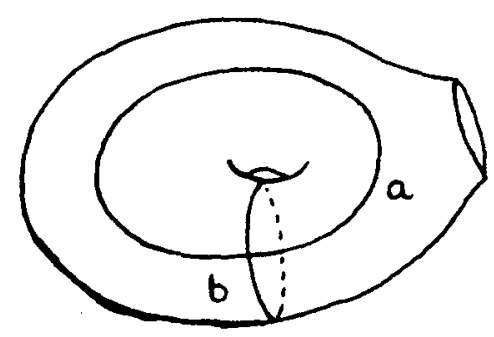


Temos que $\Pi_{1}(X)=\langle a\rangle *\langle b\rangle=G, \Pi_{1}(\partial X)=\left\langle a b a^{-1} b^{-1}\right\rangle=S$ e $(X, \partial X)$ é um par Eilenberg-Maclane que realiza $(G, S)$. Por $3.5,(G, S)$ é um $P D^{2}$-par. Logo $E(G, S)=1$, pela proposiçâo anterior.

No exemplo seguinte usamos 3.6 para concluir que um determinado par $(G, S)$ nào é de dualidade. Além disto, sabemos por 3.3 , que se $(G, S)$ é um $D^{n}$-par entào $G$ e $S$ são $D^{n-1}$-grupos. O exemplo abaixo nos mostra que a proposição 3.6 é falsa se nós substituirmos a hipótese " $(G, S)$ é um $D^{n}$-par" pela condição mais fraca " $G$ e $S$ são $D^{n-1}$-grupos". Tal exemplo é computado usando a interpretação de $E(G, S)$ em termos de derivaçôes e derivaçôes principais, dada em 2.5 .

3.8 Exemplo: Se $G=\langle a\rangle *\langle b\rangle \simeq \mathbb{Z} * \mathbb{Z}$ e $S=\langle b\rangle$ então $E(G, S)=\infty$. Observe que $G$ é um $D^{1}$-grupo, $S$ é um $P D^{1}$-grupo e no entanto, por 3.6 , $(G, S)$ não será um $D^{2}$-par.

De fato, todo elemento de $G$ pode ser unicamente escrito na forma $a^{\alpha_{1}} b^{\beta_{2}} \ldots a^{\alpha_{n}} b^{\beta_{n}}$ onde $n \geq 1, a_{i}, \beta, \in \mathbb{Z}$ e sâo não nulos, exceto possivelmente para $\alpha_{1}$ e $\beta_{n}$.

Seja $f$ um elemento de $\operatorname{Der}\left(G, S, \mathbb{Z}_{2}(G / S)\right)$. Afirmamos que $f$ depende somente de $f(a)$. Mais precisamente, temos

$$
f\left(a^{a}\right)=\sum_{i=0}^{o-1} a^{i} f(a) \quad \text { e } \quad f\left(a^{-o}\right)=\sum_{i=0}^{o} a^{-i} f(a), \quad \forall a>0 .
$$

(2) $\left\{\begin{aligned} f\left(a^{a} b^{\beta}\right) & =a^{\alpha} f\left(b^{\beta}\right)+f\left(a^{\alpha}\right)=f\left(a^{\alpha}\right) \quad \text { visto que } f_{1 S}=0, \\ f\left(a^{\alpha_{1}} b^{\beta_{1}} a^{\alpha_{2}} b^{\beta_{2}}\right) & =a^{\alpha_{1}} b^{3_{1}} f\left(a^{\alpha_{2}} b^{\beta_{2}}\right)+f\left(a^{\alpha_{1}} b^{\beta_{1}}\right)=a^{\alpha_{1}} b^{\beta_{1}} f\left(a^{\alpha_{2}}\right)+f\left(a^{\alpha_{1}}\right) \\ \text { e mais geralmente, } & \\ f\left(a^{\alpha_{1}} b^{\beta_{1}} \ldots a^{\alpha_{n}} b^{\beta_{n}}\right) & =\sum_{j=1}^{n} a^{\alpha_{1}} b^{\beta_{1}} \ldots a^{\alpha_{-1}} b^{\beta_{j-1}} f\left(a^{\alpha_{\jmath}}\right) .\end{aligned}\right.$

Estes fatos podem ser facilmente provados pelo princípio de indução finita (induçào sobre a e $n$ respectivamente). Vejamos por exemplo (1) no caso das potêncjas negativas.

(i) 0 caso $\alpha=1$ é verdadeiro, pois $0=f(1)=f\left(a^{-1} \cdot a\right)=a^{-1} f(a)+f\left(a^{-1}\right)$ 
e portanto $f\left(a^{-1}\right)=a^{-1} f(a)$.

(ii) Suponhamos que a igualdade é valida para $\alpha$, isto é, $f\left(a^{-\alpha}\right)=\sum_{i=1}^{a} a^{-i} f(a)$.

(iii) Provemos que a igualdade é verdadeira para $\alpha+1$ :

$$
\begin{aligned}
f\left(a^{-(a+1)}\right) & =f\left(a^{-a} \cdot a^{-1}\right)=a^{-\alpha} f\left(a^{-1}\right)+f\left(a^{-a}\right) \\
& \stackrel{(i)}{=} a^{-a} \cdot a^{-1} f(a)+f\left(a^{-o}\right) \\
& \stackrel{(i i)}{=} a^{-(a+1)} f(a)+\sum_{i=1}^{\alpha} a^{-i} f(a) \\
& =\sum_{i=1}^{\alpha+1} a^{-i} f(a) .
\end{aligned}
$$

Agora, para duas classes laterais de $S$, nós temos, $g_{1} S=g_{2} S$ se, e somente se, $g_{1}=g_{2} b^{\beta}$ para algum $\beta \in \mathbb{Z}$. Dai, $E:=\{1\} \cup\left\{a^{\alpha_{1}} b^{\beta_{1}} \ldots a^{\alpha_{k-1}} b^{\beta_{k-1}} a^{\alpha_{k}}\right.$ onde $k \geq 1$, $a_{j}, \beta_{j} \in \mathbb{Z}$ sâo nào nulos, exceto para $\left.a_{1}\right\}$, é um conjunto de representantes para as classes laterais à esquerda de $S$ em $G$.

Das considerações anteriores temos que todo elemento $u \in \mathbb{Z}_{2}(G / S)$ dá origem a uma derivação $f$ em $\operatorname{Der}\left(G, S, \mathbb{Z}_{2}(G / S)\right)$, basta tomarmos $f(a)=u, f(b)=0$ e estender de modo geral usando (1) e (2).

Sejam $f_{j}, j \in \mathbb{Z}$, as derjvaçōes obtidas deste modo considerando $f_{j}(a):=b^{j} a S$.

Usando o fato que $\mathbb{Z}_{2}(G / S)$ é o grupo abeliano livre gerado pelas classes laterais $g S$, com $g \in E$, podemos verificar que $f_{j}$ nào pertence a $P\left(G, S, \mathbb{Z}_{2}(G / S)\right), \forall j \in \mathbb{Z}$, e $\left\{f_{j}+P\left(G, S, \mathbb{Z}_{2}(G / S)\right): j \in \mathbb{Z}\right\}$ é um conjunto infinito de elementos linearmente independentes de $\frac{D \epsilon r\left(G, S, \mathbb{Z}_{2}(G / S)\right)}{P\left(G, S, \mathbb{Z}_{2}(G / S)\right)}$. Logo, por $2.5, E(G, S)=\infty$.

Nós finalizamos esta seç̧ão com um resultado que é útil para computarmos $E(G, S)$ no caso em que $G$ e $S$ satisfazem certas propriedades de dualidade.

3.9 Proposição: $S \epsilon j a m G$ um $D^{n}$-grupo com módulo dualizante $C \in S$ um subgrupo de $G$.

(i) Se $S$ é um $D^{n-1}$-grupo com módulo dualizante $R \epsilon s_{S}^{G} C$ (em particular, se $G$ e $S$ são grupos de dualidade de Poincaré) entâo $E(G, S) \leq 2$. 
(ii) Se hdS $\leq n-2$ entào $E(G, S)=1$.

Demonstração: Primeiro observamos que, em ambos os casos, $[G: S]=\infty$ e portanto $E(G, S)$ está definido. Isto segue de 1.5.13, 1.5.15(b) e 1.5.9(iii).

$$
\text { (i) } \begin{array}{rlr}
H^{1}\left(G ; \mathbb{Z}_{2}(G / S)\right) & \simeq H_{n-1}\left(G ; C \otimes \mathbb{Z}_{2}(G / S)\right) & \text { (por I.5.14) } \\
& \simeq H_{n-1}\left(G ; C \otimes \operatorname{Ind} d_{S}^{G} \mathbf{Z}_{2}\right) & (\text { por I.3.13(a)) } \\
& \simeq H_{n-1}\left(G ; \operatorname{Ind} d_{S}^{G}\left(\operatorname{Res}_{S}^{G} C \otimes \mathbf{Z}_{2}\right)\right) & \text { (por I.3.10) } \\
& \simeq H_{n-1}\left(S ; R \epsilon s_{S}^{G} C \otimes \mathbb{Z}_{2}\right) & \text { (por I.3.14) } \\
& \simeq H^{0}\left(S ; \mathbb{Z}_{2}\right) & \text { (por hipótese (i)) } \\
& \simeq \mathbb{Z}_{2} &
\end{array}
$$

Dai, visto que ker $r s_{S}^{G}$ é um submódulo de $H^{1}\left(G ; \mathbb{Z}_{2}(G / S)\right)$, temos que $E(G, S)=1+\operatorname{dim} \operatorname{Ker} r \epsilon s_{S}^{G} \leq 1+\operatorname{dim} H^{1}\left(G ; \mathbb{Z}_{2}(G / S)\right) \leq 2$.

(ii) Se $h d S \leq n-2$ então $H_{n-1}(S ; M)=0$ para todo $\mathbb{Z}_{2} S$-módulo $M$, em particular $H_{n-1}\left(S ; R \in s_{S}^{G} C \otimes \mathbb{Z}_{2}\right)=0$. Logo pelo mesmo raciocínio de (i) obtemos que $H^{1}\left(G ; \mathbb{Z}_{2}(G / S)\right)=0$ e portanto $E(G, S)=1$.

3.10 Exemplos: (a) Seja $G=\mathbb{Z}^{k}$ e $S=\mathbb{Z}^{r}$, com $k>r \geq 2$. Por I.5.23(d), $G$ é um $P D^{k}$-grupo e $S$ é um $P D^{\top}$-subgrupo. Daí, por $3.9, E(G, S) \leq 2$ se $r=k-1$ e $E(G, S)=1$ se $r \leq k-2$.

(b) Se $G=\Pi_{3}\left(K^{\prime} B\right)=\left\langle a, b ; a^{2} b^{2}=1\right\rangle$ e $S=\langle a\rangle$, então $E(G, S) \leq 2$.

(c) Seja $G=(\mathbb{Z} \oplus \mathbb{Z}) \rtimes \mathbb{Z}$, onde $\theta: \mathbb{Z} \rightarrow \operatorname{Aut}(\mathbf{Z} \oplus \mathbb{Z})$ é dada por

$$
\theta(c)(a, b)=\left[\begin{array}{ll}
1 & 0 \\
2 & 1
\end{array}\right]^{c}\left[\begin{array}{l}
a \\
b
\end{array}\right]=\left[\begin{array}{cc}
1 & 0 \\
2 c & 1
\end{array}\right]\left[\begin{array}{l}
a \\
b
\end{array}\right]=(a, 2 c a+b)
$$

A operação em $G$ é dada por

$$
((a, b) . c)+\left(\left(a_{1}, b_{1}\right), c_{1}\right)=\left((a, b)+\theta(c)\left(a_{1}, b_{1}\right), c+c_{1}\right)=\left(a+a_{1}, b+b_{1}+2 c a_{1}, c+c_{1}\right) .
$$

Temos que $G$ é um $P D^{3}$-grupo, pois $G$ é o grupo fundamental de uma variedade asférica de dimensâo 3 (cf. [23]).

Seja $S=\{((a, b), 0) ; a, b \in \mathbb{Z}\}$. Temos que $S$ é um subgrupo (normal) de $G$ isomorfo a $\mathbb{Z} \oplus \mathbb{Z}$. Dai $S$ é um $P D^{2}$-subgrupo e portanto $E(G, S) \leq 2$. 
Analogamente, se considerarmos $K=\{((0, b), c) ; b, c \in \mathbf{Z}\}$, temos que $K$ é um subgrupo (abeliano e normal) de $G$ e portanto também $E(G, K) \leq 2$.

(d) Se $G$ é como no exemplo anterior e $S=\{((0,0), c) ; c \in \mathbb{Z}\}$ então $S$ é um subgrupo de $G$ (não normal). Como $S$ é um $P D^{1}$-subgrupo, segue de 3.9 (ii) que $E(G, S)=1$. Se $G^{\prime}$ é qualquer extensào finita de $G$, entào também $E\left(G^{\prime}, S\right)=1$ pois, por 2.12, $E\left(G^{\prime}, S\right) \leq E(G, S)$.

Nota: De fato, concluiremos (na seç̧âo seguinte) que em (a), $E\left(\mathbf{Z}^{k}, \mathbb{Z}^{k-1}\right)=2$ e em $(\mathrm{c}), E(G, S)=E\left(G, K^{\prime}\right)=2$.

\section{4. $E(G, S)$ e o Invariante end $e(G, S)$.}

Os principais resultados desta seç̧âo surgiram da tentativa em dar uma interpretação para o invariante end $\epsilon(G, S)$ de Houghton [17] e Scott [26], em termos de cohomologia relativa. Na realidade grande parte deste trabalho teve sua origem aqui.

Com o propósito de situar o leitor na teoria de ends e evitar frequentes consultas às referências, apresentamos, inicialmente, uma breve revisão dos conceitos e resultados básicos: (sem demonstraşào) da teoria de ends de grupos $e(G)$ e ends de pares de grupos $e(G, S)$. Para majores detalhes ver [9], [26] e [2i].

\section{Ends de Grupos}

A teoria de ends de grupos teve sua origem na teoria de ends de espaço. A definição de ends de espaço foi introduzida por Freudenthal [13] (1931). Para um estudo do conjunto de ends de $X$, onde $X$ é um espaço topológico, nós sugerimos [29]. Aqui estamos interessados somente no número de ends, nâo necessariamente no conjunto de ends.

4.1 Definição: Seja $X$ um complexo simplicial localmente finito. Para todo 
subcomplexo $K$, o número de componentes de $X-K$ é finito. Seja $n(K)$ o número de componentes infinitas (ou equivalentemente, cujo fecho é nào compacto), então o número de ends de $X$ é definido por:

$$
e(X)=\sup \left\{n(K), K \subseteq X, K^{\prime} \text { finito }\right\}
$$

Nota: Esta definição vale igualmente para um $C W$-complexo localmente finito, se nós simplesmente trabalharmos com células ao invés de simplexos.

\subsection{Exemplos:}

(i) $e(X)=0 \Leftrightarrow X$ é um complexo simplicial finito (ou equivalentemente, $X$ é compacto).

(ii) $\epsilon(\mathbb{R})=2$ pois, para qualquer compacto $K$, existe um intervalo fechado $I$ tal que $K \subset I$. Claramente $\mathbb{R}-I$ tem duas componentes $C_{1}, C_{2}$, ambas infinitas, e uma componente de $\mathbb{R}$ - $h$ que nào intercepta nenhuma destas componentes está inteiramente contida em $I$ e portanto é finita.

Nós ilustramos o caso em que $K$ tem duas componentes conexas:

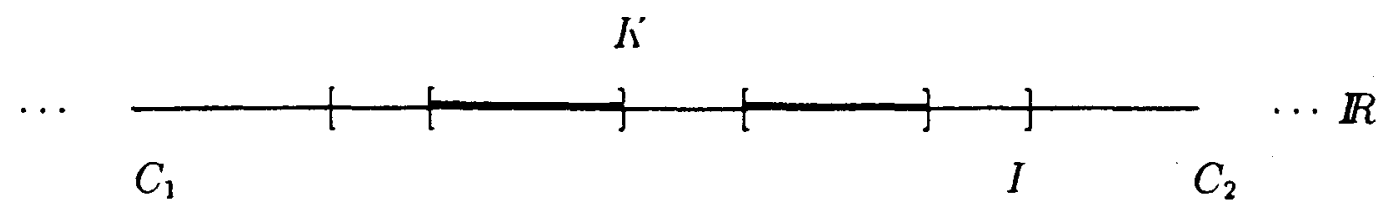

(iii) $\epsilon\left(\mathbb{R}^{n}\right)=1$ se $n \geq 2$.

Hopf em [16] (1943) mostrou que se um grupo finitamente gerado $G$ atua livremente sobre um complexo simplicial $\hat{X}$ tal que o quociente $\hat{X} / G$ é um complexo simplicial finito entào $\epsilon(\hat{X})$ depende somente de $G$. Assim, no caso em que $G$ é finitamente gerado, podemos definir o número de ends de $G$ como sendo $\epsilon(\hat{X})$.

Foi Specker em [30] (1949) quem apresentou uma definição de número de ends de $G$, para qualquer grupo $G$ (nâo necessariamente finitamente gerado). Sua definição é puramente algébrica. Antes de introduzirmos a definição faremos algumas consideraçōes. 
4.3: Seja $G$ um grupo. Por I.3.7, $\mathbb{Z}_{2} G$ pode ser considerado como um $\mathbf{Z}_{2} G$. submódulo de $\left.\overline{\mathbf{Z}_{2} G}=\operatorname{Coind} G\right\} \mathbb{Z}_{2}$. Denotemos por $E G$ o $\mathbf{Z}_{2} G$-módulo quociente $\frac{\overline{\mathbf{Z}_{2} G}}{\mathbf{Z}_{2} G}$.

Conforme vimos em 1.3.15, existe um $\mathbb{Z}_{2} G$-isomorfismo $\rho: \overline{Z_{2} G} \rightarrow P G$. Considere $F G=\{A \subset G \mid A$ é finito $\}$. É fácil verificar que a imagem do $\mathbf{Z}_{2} G$-monomorfismo

$$
\mathbb{Z}_{2} G \stackrel{\oplus}{\hookrightarrow} \overline{\mathbb{Z}_{2} G} \stackrel{\rho}{\rightarrow} P G
$$

é exatamente $F G$. Em outras palarras, o submódulo $\mathbb{Z}_{2} G$ de $\overline{\mathbf{Z}_{2} G}$ corresponde via o isomorfismo $\rho$, ao submódulo $F G$ de $P G$. Assim temos o $\mathbb{Z}_{2} G$-isomorfismo

$$
E G=\frac{\overline{\mathbb{Z}_{2} G}}{\mathbb{Z}_{2} G} \simeq \frac{P G}{F G} .
$$

4.4 Definição: $O$ número de ends $d \epsilon G$ é definido como

$$
\epsilon(G)=\operatorname{dim} H^{0}(G ; E G)=\operatorname{dim}\left(\frac{P G}{F G}\right)^{G} .
$$

No caso em que $G$ é finitamente gerado este número $e(G)$ (obtido de forma algébrica) é igual ao número de ends de um espaşo topológico construido a partir de $G$ como segue:

Suponhamos que $G$ é gerado por $U=\left\{x_{1} \ldots, x_{n}\right\}$ e seja $\Gamma_{U}$ o diagrama $d \epsilon$ Cayley correspondente para $G$. Isto é, $\Gamma_{U}$ tem como vértices os elementos de $G$ e para todo $i$ existe uma aresta ligando os pontos $g$ a $x_{i} g$. Note que claramente $G$ atua à direita sobre $\Gamma_{U}$.

4.5 Proposição: [2خ, prop. 5.2] Se $G$ é gerado por $U=\left\{x_{1}, \ldots, x_{n}\right\}$ entào $\epsilon(G)=\epsilon\left(\Gamma_{U}\right)$.

4.6 Corolário: (i) $\epsilon(\mathbb{Z})=2$ pois $U=\{1\}$ gera $\mathbb{Z} \in \Gamma_{\{1\}}=\mathbb{R}$.

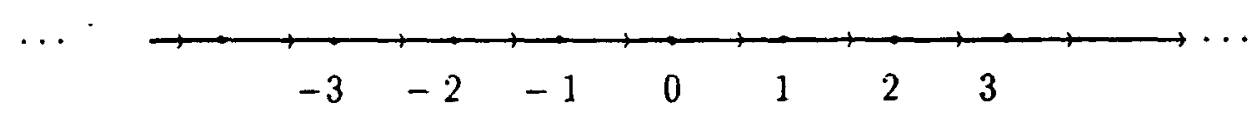


(ii) $\varepsilon\left(\mathbf{Z}_{\mathbf{2}} * \mathbf{Z}_{\mathbf{2}}\right)=2$.

(iii) $\epsilon(\mathbf{Z} * \mathbf{Z})=\infty$

Esta conexão pode ser estendida.

4.7 Proposição: [26, lema 1.2] Se $G$ atua livremente sobre um complexo contro $\dot{X}$, com quociente finito $X$ (equivalentemente, $\dot{X} \rightarrow X$ é um recobrimento regular conexo, com grupo de recobrimento $G$ ), entāo $e(G)=\epsilon\left(\hat{X}^{\prime}\right)$.

Observe que o quociente $X$ sendo finito implica que $G$ é finitamente gerado, e portanto o resultado anterior confirma que no caso em que $G$ é finitamente gerado, a definição dada por Hopf para o número de ends de $G$ coincide com a definição algébrica dada por Specker.

4.8 Corolário: $S \in G$ atua livremente no $\mathbb{R}^{n}, n>1$, com quociente compacto $\epsilon$ ntão $\epsilon(G)=1$. Em particular $\epsilon\left(\mathbb{Z}^{k}\right)=1$ se $k \geq 2$.

No lema seguinte nós reunimos algumas propriedades de $e(G)$.

4.9 Lema: $[27]$

(i) $\epsilon(G)=0 \Leftrightarrow G$ '́ finito.

(ii) Sc $S$ ć um subgrupo d€ $G$ tal que $[G: S]<\infty$ entâo $\epsilon(G)=\epsilon(S)$.

(iii) $S \epsilon S$ é um subgrupo finito normal de $G$ entâo $\epsilon(G)=\epsilon(G / S)$.

Se $G$ é qualquer grupo infinito, nós temos a seguinte interpretaçâo de $\epsilon(G)$ em termos de cohomologia de grupos.

4.10 Proposição: $S \epsilon G$ t́ um grupo infinito entâo

$$
\epsilon(G)=1+\operatorname{dim} H^{1}\left(G ; \mathbb{Z}_{2} G\right)
$$

4.11 Corolário: $S \epsilon G \epsilon^{\prime}$ um $D^{n}$-grupo, $n>1$, entâo $\epsilon(G)=1$. 
Um resultado interessante que, no caso em que $S$ é finitamente gerado, pode ser visto em [27, corol. 5.9] é o seguinte:

4.12 Proposição: Seja $G$ um grupo. Então $e(G)=0,1,2$ ou $\infty$.

Existe uma classificação completa para grupos com dois ends (devida a Hopf, 1943).

4.13 Teorema: [27, teor. 5.12] As seguintes condiçōes são equivalentes para grupos finitamente gcrados:

(i) $\epsilon(G)=2$.

(ii) $G$ tem um subgrupo cíclico infinito de índice finito.

(iii) $G$ tem um subgrupo normal $\boldsymbol{h}$ tal que $G / K$ é isomorfo a $\mathbb{Z}$ ou ao grupo diedral infinito $D_{\infty}=\mathbb{Z}_{2} * \mathbb{Z}_{2}$.

$O$ end $e(G, S)$

O conceito de número de ends $\epsilon(G, S)$ de um par grupo $(G, S)$, onde $S$ é um subgrupo de $G$, é uma genẹralizaçâo do número de ends de um grupo.

A definição natural de $\epsilon(G, S)$ é derida a Houghton [17] (1974) e foi estabelecida para grupos topológicos. Scott em [26] (1977), explorou este invariante para grupos discretos.

De modo similar a 4.3 , podemos, por 1.3 .13 , considerar $\mathbb{Z}_{2}(G / S)$ como um $\mathbb{Z}_{2} G$-submódulo de $\overline{\mathbb{Z}_{2}(G / S)}$, e o $\mathbb{Z}_{2} G$-módulo quociente $E(G / S)=\frac{\overline{\mathbb{Z}_{2}(G / S)}}{\mathbb{Z}_{2}(G / S)} \simeq$ $\frac{P(G / S)}{F(G / S)}$

4.14 Definição: Sejam $G$ um grupo $\epsilon S$ um subgrupo de G. Então: por $d \epsilon$ finiçâo,

$$
\epsilon(G, S)=\operatorname{dim} H^{0}(G ; E(G / S))=\operatorname{dim}\left(\frac{P(G / S)}{F(G / S)}\right)^{G}
$$


No lema seguinte agrupamos algumas propriedades de $\epsilon(G, S)$. Elas estào contidas em [26]

\subsection{Lema:}

(i) $\epsilon(G,\{1\})=\epsilon(G)$.

(ii) $\epsilon(G, S)=0 \Leftrightarrow[G: S]<\infty$.

(iii) Se $S \subset T \subset G$, com $[G: T]<\infty$ entào $\epsilon(G, S)=\epsilon(T, S)$.

(iv) Se $S$ é um subgrupo normal cm $G$, entâo $\epsilon(G, S)=\epsilon(G / S)$.

(v) Sejam $A \in S$ grupos nào triviais. $S \in A=S=\mathbb{Z}_{2}$ entào $\epsilon(A * S, S)=1$, caso contrário $\epsilon(A * S, S)=\infty$.

(vi) Se $G$ é um grupo livrc e $S$ é um subgrupo finitamente gerado de $G$ tal que $[G: S]=\infty$ entâo $\epsilon(G, S)=\infty$.

O invariante $e(G, S)$ também está relacionado com espaços topológicos como nos mostra o resultado abaixo, que é uma extensão das proposiçôes 4.5 e 4.7 (cf. $[26$, lemas 1.1 e 1.2$])$.

\subsection{Proposição:}

(i) Scjam $G$ um grupo finitamente gerado, $S$ um subgrupo de $G \in \Gamma$ o diagrama de Cayley associado a $G$ como em 4.5. Se $\Gamma / S$ denota o quociente de $\Gamma$ pela asáo à direita de $S$ entâo $\epsilon(G, S)=\epsilon(\Gamma / S)$.

(ii) Se $X$ é um CW -complexo finito com espaço de recobrimento regular conexo $\hat{X}$ cujo grupo de recobrimento $\dot{\epsilon} G$ e $S$ é um subgrupo de $G$, entâo $\epsilon(G, S)=$ $\epsilon(\hat{X} / S)$, onde $\hat{X} / S$ é o quociente de $\hat{X}$ pela aşâo do subgrupo $S$.

É válido para $(G, S)$ um resultado similar ao 4.12 acima, porém com alguma restriçào.

4.17 Proposição: [17, teor. 4.1] Se $G \epsilon S$ são finitamente gerados $\epsilon$ $\left[N_{G}(S): S\right]=\infty$, onde $\Lambda_{G}(S)$ denota o normalizador de $S$ em $G$, então $\epsilon(G, S)=$ 1,2 , ou $\infty$. 
De modo geral $e(G, S)$ pode assumir qualquer valor $n \in \mathbb{N}$, como nos mostra o resultado seguinte.

4.18 Proposição: [26, lema 2.2] Seja $G$ o grupo fundamental de uma superfície fechada $F$ e sejn $S$ o grupo fundamental de uma subsuperfície compacta incompressivel $X$ de $F$. Entào $\epsilon(G, S)$ é igual ao número de componentes de bordo de $X$.

Recordamos que um circulo $C$ mergulhado em uma superficie $F$ é incompressivel se a aplicação natural $\Pi_{1}(C) \rightarrow \Pi_{1}(F)$ é injetiva; e uma subsuperfície compacta $X$ em uma superfície $F$ é incompressível se toda componente de bordo de $X$ é incompressível em $F$.

4.19 Exemplo: Seja $G$ o grupo fundamental de uma superfície fechada orientável $F$ de genus 3 , e seja $S$ o grupo fundamental da subsuperfície incompressivel $X$ de $F$ como na figura abaixo. Entào $e(G, S)=5$.

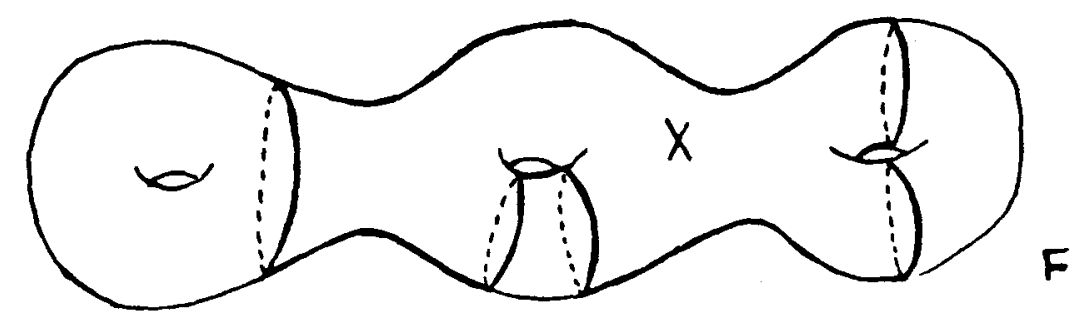

Agora estamos em condiçôes de analisar a relação entre os dois invariantes $E(G, S)$ e $e(G, S)$

Conforme inicialmente observamos, os resultados desta secção surgiram da tentativa em dar uma interpretaçâo para $\epsilon(G, S)$ em termos de cohomologia relativa.

4.20: Seja $G$ um grupo infinito entào. por 4.10 e 2.5, temos

$$
\begin{aligned}
\epsilon(G) & =1+\operatorname{dim} H^{1}\left(G ; \mathbb{Z}_{2} G\right) \\
& =1+\operatorname{dim} \frac{D c r\left(G, \mathbb{Z}_{2} G\right)}{P\left(G, \mathbb{Z}_{2} G\right)}=E(G,\{1\})
\end{aligned}
$$


Agora uma vez que $\epsilon(G,\{1\})=\epsilon(G)$, segue de 2.5 , que

$$
e(G,\{1\})=E(G,\{1\})=1+\operatorname{dim} \frac{H^{1}\left(G,\{1\} ; \mathbf{Z}_{2} G\right)}{P\left(G,\{1\}, \mathbf{Z}_{2} G\right)} .
$$

Nós notamos que para $\epsilon(G, S)$ onde $S$ é um subgrupo nào trivial de $G$ com $[G: S]=\infty$, não existe uma fórmula análoga (usando cohomologia relativa). Nossa esperança inicial era provar que $\epsilon(G, S)=E(G, S)$ e assim, por 2.5, obter uma fórmula similar para $e(G, S)$. Mas isto é falso em geral, como mostra o exemplo seguinte:

4.21 Exemplo: Se $G=\langle a\rangle *\langle b\rangle \simeq Z * Z$ e $S=\left\langle a b a^{-1} b^{-1}\right\rangle$ então $E(G, S)=1<\epsilon(G, S)=\infty$.

De fato, que $E(G, S)=1$ foi visto no exemplo 3.7 , e que $e(G, S)=\infty$ segue de 4.15 (vi).

Entretanto, provaremos no teorema 4.24 abaixo, que a desigualdade $E(G, S) \leq \epsilon(G, S)$ é sempre verdadeira, e que a igualdade ocorre quando $S$ é normal em $G$. Isto foi motivado nos seguintes exemplos:

4.22 Exemplo: Se $G=\mathbb{Z} \oplus \mathbb{Z}=\langle(t, 1) \cdot(1, s)>$ e $S=\{1\} \oplus \mathbb{Z}=$ $<(1, s)>\simeq \mathbb{Z}$. Então

$$
e(G, S)=E(G, S)=1+\operatorname{dim} \frac{H^{3}\left(G, S: \mathbb{Z}_{2}(G / S)\right)}{P\left(G, S, \mathbb{Z}_{2}(G / S)\right)}=2 .
$$

Como $S$ é normal em $G$, segue de 4.15 (iv) e 4.6 , que $\epsilon(G, S)=\epsilon(G / S)=$ $e(\mathbb{Z})=2$. Daí temos (por 3.10 (a)) a desigualdade $E(G, S) \leq 2=e(G, S)$. Para provar a igualdade usamos a interpretaçào de $E(G, S)$ em termos de derivaçooes e derivaçôes principais (lema 2.5), isto é, mostraremos que

$$
\frac{\operatorname{D\epsilon r}\left(G, S, \mathbb{Z}_{2}(G / S)\right)}{P\left(G, S, \mathbb{Z}_{2}(G / S)\right)} \simeq \mathbb{Z}_{2}
$$

Por simplicidade, denotaremos a classe $(t .1) S$ por $\mathbf{t}$, e assim, $G / S=\langle\mathbf{t}\rangle$. Os seguintes passos fornecem a prova.

1- Uma derivaçào $f \in \operatorname{Der}\left(G, S, \mathbb{Z}_{2}(G / S)\right)$. somente depende de $f(t, 1)$, 
pois, para todo $k>0$ e $j \in \mathbf{Z}$, nós temos

$$
\begin{aligned}
& f\left(t^{k}, s^{j}\right)=f\left(t^{k}, 1\right)=\sum_{i=0}^{k-1}\left(t^{i}, 1\right) f(t, 1), \quad \text { e } \\
& f\left(t^{-k}, s^{j}\right)=f\left(t^{-k}, 1\right)=\sum_{i=1}^{k}\left(t^{-i}, 1\right) f(t, 1) .
\end{aligned}
$$

(lsto pode ser provado por indução sobre k.)

2- Se $f(t, 1)=\mathbf{t}^{k}+1$ entào $f \in P\left(G, S, \mathbf{Z}_{2}(G / S)\right)$.

De fato, para $k>0$, temos $f=d_{m}$ onde $m=\mathrm{t}^{k-1}+\mathrm{t}^{k-2}+\ldots+1 \in \mathbf{Z}_{2}(G / S)$.

Para $k=-n<0$, nós temos $f=d_{m}$ onde $m=\mathrm{t}^{-n}+\mathrm{t}^{-n+1}+\cdots+\mathrm{t}^{-1}$. O caso $k=0$ é obvio.

3- Suponhamos que $f(t, 1)=\mathbf{t}^{i_{1}}+\cdots+\mathbf{t}^{i_{r}}$, com $i_{j} \neq i_{k}$, para todo $0 \leq j \neq k \leq r$. Se $r \equiv 0(\bmod 2)$ entào $f \in P\left(G, S, \mathbb{Z}_{2}(G / S)\right)$. Se $r \equiv 1(\bmod 2)$ então $f-\psi \in P\left(G, S, \mathbb{Z}_{2}(G / S)\right)$, onde $\xi^{\prime} \in \operatorname{Der}\left(G, S, \mathbb{Z}_{2}(G / S)\right)$ é dada por $\psi(t, 1)=\mathbf{1}$ e $\psi \notin P\left(G, S, \mathbb{Z}_{2}(G / S)\right)$.

A prova disto usa 1 e 2 notando que $f(t, 1)=\left(t^{i_{1}}+1\right)+\cdots+\left(t^{i_{r}}+1\right)+r .1=$ $d_{m}(t, 1)+r .1$.

4- Finalizando nós definimos o isomorfismo

$$
\begin{aligned}
\eta: \frac{\operatorname{Der}\left(G, S, \mathbb{Z}_{2}(G / S)\right)}{P\left(G, S, \mathbb{Z}_{2}(G / S)\right)} \rightarrow \mathbb{Z}_{2} \\
f+P\left(G, S, \mathbb{Z}_{2}(G / S)\right) \rightarrow \varepsilon(f(t, 1)),
\end{aligned}
$$

onde $\varepsilon: \mathbb{Z}_{2}(G / S) \rightarrow \mathbb{Z}_{2}$ é a aplicaçào dada no início do capitulo, isto é, que aplica todo gera.dor $g S$ em 1 .

Analogamente, nós temos

\subsection{Exemplos:}

(i) Se $G=\mathbb{Z}^{k}, S=\mathbb{Z}^{k-1}, k \geq 3$ entào $E(G, S)=\epsilon(G, S)=2$

(ii) Se $G=\mathbb{Z} \oplus \mathbb{Z}_{n}$ e $S=\mathbb{Z}_{n}, n>1$ onde $\mathbb{Z}_{n}$ é o grupo cíclico de ordem finita $n$, entào $E(G, S)=\epsilon(G, S)=2$. 
4.24 Teorenza: $S c G$ é um grupo es $S \dot{u}$ um subgrupo de $G \operatorname{com}[G: S]=\infty$, então $E(G, S) \leq \epsilon(G, S)$.

Alćm disto, se $S$ é normal em $G$, a igualdade ocorre, ou seja,

$$
\epsilon(G, S)=E(G, S)=1+\operatorname{dim} \frac{H^{1}\left(G, S ; \mathbb{Z}_{2}(G / S)\right)}{P\left(G, S ; \mathbb{Z}_{2}(G / S)\right)}
$$

Para demonstrarmos o teorema necessitamos do seguinte lema elementar, que nos dá uma condiçâo necessária e suficiente para que $e(G, S)=E(G, S)$.

4.25 Lema: $S \epsilon j a(G, S)$ um par grupo com $[G: S]=\infty$.

(i) Se $\varphi^{*}: H^{1}\left(G ; \mathbb{Z}_{2}(G / S)\right) \rightarrow H^{1}\left(G ; \overline{\mathbb{Z}_{2}(G / S)}\right)$ é a aplicaçâo induzida do $\mathbb{Z}_{2} G$-monomorfismo natural $\varphi: \mathbb{Z}_{2}(G / S) \rightarrow \overline{\mathbb{Z}_{2}(G / S)}$ (dado $\epsilon m$ I.3.13), então

$$
\epsilon(G, S)=1+\operatorname{dim} \operatorname{Ricr} \varphi^{*}
$$

(ii) $E(G, S)=e(G, S) \Leftrightarrow \operatorname{dim} \operatorname{Ker} r \epsilon s_{S}^{G}=\operatorname{dim} \operatorname{Ker} \varphi^{*}$, onde $r \in s_{S}^{G}: H^{1}\left(G ; \mathbb{Z}_{2}(G / S)\right) \rightarrow H^{1}\left(S ; \mathbb{Z}_{2}(G / S)\right)$ é a aplicaçâo restriçâa.

Demonstração: (ii) é uma consequencia imediata de (i) e da definiçâo de $E(G, S)$. Vejamos (i):

A sequência exata curta

$$
0 \rightarrow \mathbb{Z}_{2}(G / S) \stackrel{\leftrightarrow}{\rightarrow} \overline{\mathbb{Z}_{2}(G / S)} \rightarrow E(G, S)=\frac{\overline{\mathbb{Z}_{2}(G / S)}}{\mathbb{Z}_{2}(G / S)} \rightarrow 0,
$$

induz (ver I.2.2(ii)') a sequencia exata longa

$$
\begin{gathered}
0 \rightarrow H^{0}\left(G: \mathbb{Z}_{2}(G / S)\right) \rightarrow H^{0}\left(G ; \overline{\mathbb{Z}_{2}(G / S)}\right) \rightarrow H^{0}(G ; E(G / S)) \stackrel{\wp}{\rightarrow} \\
\rightarrow H^{1}\left(G ; \mathbb{Z}_{2}(G / S)\right) \stackrel{\stackrel{\bullet}{\bullet}^{\rightarrow}}{\rightarrow} H^{1}\left(G ; \overline{\mathbb{Z}_{2}(G / S)}\right) \rightarrow \cdots
\end{gathered}
$$

Agora, $H^{0}\left(G ; \mathbb{Z}_{2}(G / S)\right)=0$ visto que $[G: S]=\infty$ (1.3.11) e $H^{0}\left(G ; \overline{\mathbb{Z}_{2}(G / S)}\right) \simeq H^{0}\left(S ; \mathbb{Z}_{2}\right) \simeq \mathbb{Z}_{2}$ por 1.3.17. Daí nós temos a sequencia exata curta

$$
0 \rightarrow \mathbb{Z}_{2} \rightarrow H^{0}(G ; E(G / S)) \rightarrow \operatorname{Ker} \varphi^{*} \rightarrow 0
$$


e portanto (ver 1.14), $e(G, S)=1+\operatorname{dim} \operatorname{Ker} \varphi^{*}$, como desejado.

Demonstração do teorema 4.24: Seja $A=\varphi\left(Z_{2}(G / S)\right)$ onde $\varphi: \mathbb{Z}_{2}(G / S) \rightarrow \overline{\mathbb{Z}_{2}(G / S)}$ é como no lema acima. Temos que $\mathbf{Z}_{2}(G / S)$ é $\mathbf{Z}_{2} G$-isomorfo (via $\varphi$ ) ao submódulo $A$ de $\overline{\mathbf{Z}_{2}(G / S)}$ e portanto temos os isomorfismos

$$
H^{1}\left(G ; \mathbb{Z}_{2}(G / S)\right) \stackrel{\dot{\oplus}}{\rightarrow} H^{1}(G, A), \quad \text { e } \frac{\operatorname{Der}\left(G, S, \mathbb{Z}_{2}(G / S)\right)}{P\left(G, S, \mathbf{Z}_{2}(G / S)\right)} \simeq \frac{\operatorname{Der}(G, S, A)}{P(G, S, A)}
$$

Daj

$$
E(G, S)=1+\operatorname{dim} \frac{\operatorname{Der}(G, S, A)}{P(G, S, A)} .
$$

Sejam $\alpha: S \hookrightarrow G$ e $k: A \hookrightarrow \overline{\mathbb{Z}_{2}(G / S)}$ as inclusões (de grupos e módulos, respectivamente), e $\pi^{\prime}$ a composiçào das aplicaçôes $A \stackrel{k}{\leftrightarrow} \overline{\mathbb{Z}_{2}(G / S)} \stackrel{v}{\rightarrow} \operatorname{Coind}_{S}^{G} \mathbb{Z}_{2} \stackrel{\pi}{\rightarrow} \mathbb{Z}_{2}$, onde $v$ é o $\mathbb{Z}_{2} G$-isomorfismo dado em 1.3 .13 e $\pi$ é o $\mathbb{Z}_{2}$ S-epimorfismo dado em 1.3.4. Assim $\pi^{\prime}(l)=v(l)(1)=l(1 S), \forall l \in A \subset \operatorname{Hom}\left(\mathbb{Z}_{2}(G / S), \mathbb{Z}_{2}\right)$.

Consideremos as aplicaçôes induzidas ( 1 , secção 2)

$$
H^{1}\left(G ; \mathbb{Z}_{2}(G / S)\right) \stackrel{\varphi^{*}}{\rightarrow} H^{1}(G ; A) \stackrel{k^{*}}{\rightarrow} H^{1}\left(G ; \overline{\mathbb{Z}_{2}(G / S)}\right) \stackrel{\bullet^{*}}{\rightarrow} H^{1}\left(G ; \operatorname{Coind}_{S}^{G} \mathbb{Z}_{2}\right) \stackrel{(a, \pi)^{*}}{\rightarrow} H^{1}\left(S ; \mathbb{Z}_{2}\right) .
$$

Como $\dot{\varphi}, v^{*}$ e $(a, \pi)^{*}$ sào isomorfismos. temos

$$
\text { Ker } \varphi^{*} \simeq \operatorname{lier} k^{*}=\operatorname{lier} t \text {. }
$$

Por outro lado, o homomorfismo

$$
\psi: \frac{\operatorname{Der}(G, S, A)}{P(G, S, A)} \rightarrow \frac{\operatorname{Der}(G, A)}{P(G, A)}=H^{1}(G ; A)
$$

definido por $\psi(f+P(G, S, A))=[f]=f+P(G, A)$ é um monomorfismo e daj́

$$
\frac{\operatorname{Dcr}(G, S, A)}{P(G, S, A)} \simeq \operatorname{Im} \psi^{\prime \prime}=\{[f] \mid f \in \operatorname{D\epsilon r}(G . S, A)\}
$$

Logo, para provar a primeira parte do teorema é suficiente provar que

$$
\operatorname{In} \psi \subset \text { lier } t
$$

pois dai, temos: 


$$
\begin{array}{rlrl}
E(G, S) & =1+\operatorname{dim} \frac{\operatorname{Dcr}(G, S, A)}{P(G, S, A)} & & \text { (por (1)) } \\
& =1+\operatorname{dim} \operatorname{Im} \psi & & \text { (por (3)) } \\
& \leq 1+\operatorname{dim} \operatorname{Ker} t & & \text { (por (4)) } \\
& =1+\operatorname{dim} \operatorname{Ker} \varphi^{*} & \text { (por (2)) } \\
& =e(G, S) & & \text { (pelo lema anterior) }
\end{array}
$$

Provemos entâo (4): Temos que $t$ é a aplicaçào induzida de $\left(\alpha, \pi^{\prime}\right)$. Sejam $P \rightarrow \mathbf{Z}_{2}$ e $F \rightarrow \mathbf{Z}_{2}$ as resoluçōes Bar para $S$ e $G$ respectivamente (como em $\operatorname{I.14}(\mathrm{c})$ )). Claramente a inclusào $\tau: P \rightarrow F$ é uma aplicação de cadeia compatível com $\alpha$. Daí temos o seguinte diagrama

$$
\begin{aligned}
& 0 \rightarrow C^{0}(G, A) \quad \stackrel{\delta^{3}}{\rightarrow} \quad C^{1}(G, A) \quad \stackrel{\delta^{2}}{\rightarrow} \ldots \\
& \downarrow \quad \downarrow \operatorname{Hom}\left(\tau, \pi^{\prime}\right) \\
& 0 \rightarrow C^{0}\left(S, \mathbb{Z}_{2}\right) \stackrel{\overline{\delta^{1}}}{\rightarrow} \quad C^{1}\left(S, \mathbb{Z}_{2}\right) \quad \rightarrow \ldots
\end{aligned}
$$

onde $\operatorname{Hom}\left(\tau, \pi^{\prime}\right)(f)=\pi^{\prime} \circ f \circ \tau$ para todo $f \in C^{1}(G, A)$, e $t: H^{1}(G ; A) \rightarrow H^{1}\left(S ; \mathbb{Z}_{2}\right)$ é tal que $t[f]=\left(\alpha, \pi^{\prime}\right)^{-}([f])=\left[\pi^{\prime} \circ f \circ \tau\right]$.

Seja $[f] \in \operatorname{Im} \psi \subset H^{1}(G, A)$. Como $f \in \operatorname{Der}(G, S, A)$ temos que $f_{\mid S}=0$. Daí $\pi^{\prime} \circ f \circ \tau: S \rightarrow \mathbb{Z}_{2}$ satisfaz $\left(\pi^{\prime} \circ f \circ \tau\right)(s)=\pi^{\prime}(f(s))=f(s)(1 S)=0$. Logo $t[f]=\left[\pi^{\prime} \circ f \circ \tau\right]=0$ e assim Im $\psi$ ' C Ker $t$.

Suponhamos agora que $S$ é normal em $G$. Entào a açào de $S$ em $\mathbb{Z}_{2}(G / S)$ é trivial $\left(s . g S=s g S=g\left(g^{-1} s g\right) S=g S\right)$.

Usando este fato provaremos que

Ker $k^{*} \subset \operatorname{Im} \psi=\{[f] \mid f \in D \in r(G, S, A)\}$

dai teremos $e(G, S) \leq E(G, S)$, que junto com a primeira parte do teorema nos fornece a igualdade desejada.

Provemos (5): Seja $F \rightarrow \mathbb{Z}_{2}$ a resolução Bar para $G$ e considere o seguinte diagrama

$$
\begin{aligned}
& 0 \rightarrow \quad C^{0}(G, A) \quad \stackrel{\delta^{1}}{\rightarrow} \quad C^{1}(G, A) \quad \stackrel{\delta^{2}}{\rightarrow} \ldots \\
& \downarrow \\
& 0 \rightarrow C^{0}\left(G, \overline{\mathbb{Z}_{2}(G / S)}\right) \stackrel{\overline{\delta^{1}}}{\rightarrow} C^{1}\left(G, \overline{\mathbb{Z}_{2}(G / S)}\right) \stackrel{\overline{\mathcal{E}^{2}}}{\rightarrow} \ldots
\end{aligned}
$$


onde $k^{*}(f)=k \circ f$. Temos que a induzida $k^{*}: H^{1}(G ; A) \rightarrow H^{1}\left(G ; \mathbb{Z}_{2}(G / S)\right)$ é tal que $k^{*}[f]=\left[k^{*}(f)\right]=[k \circ f]$.

Seja $[f] \in \operatorname{Ker} k^{*} \subset H^{1}(G ; A)$. Como $f \in \operatorname{Ker} \delta^{2}=\operatorname{Dcr}(G, A)$, para provar (5) basta provar que $f_{\mid S}=0$ ou seja, $f(s) \in A \subset \operatorname{Hom}\left(\mathbb{Z}_{2}(G / S), \mathbf{Z}_{2}\right)$ é a aplicaçào nula para todo $s \in S$. Mas $k^{*}[f]=0$ implica que $k \circ f \in \operatorname{Im} \overline{\delta^{1}}$ e portanto existe $u \in C^{0}\left(G, \overline{\mathbf{Z}_{2}(G / S)}\right)$ tal que $k \circ f=\overline{\delta^{1}}(u)$. Logo $f(g)=(k \circ f)(g)=\overline{\delta^{1}}(u)(g)=g \cdot u[]-u[]$ para todo $g \in G$, em particular, $f(s)=s u[]-u[], \forall s \in S$. Daí, usando o fato que as açồes de $S$ em $\mathbb{Z}_{2}$ e $\mathbb{Z}_{2}(G / S)$ sào triviais, temos que, para qualquer $\bar{g}=g S \in \mathbb{Z}_{2}(G / S)$, $f(s)(\bar{g})=s . u[](\bar{g})-u[](\bar{g})=s u[]\left(s^{-1} \bar{g}\right)-u[] \bar{g}=u[](\bar{g})-u[](\bar{g})=0$, isto é, $f_{\mid S}=0$, como queríamos.

4.26: Sejam $G, S$ e $h^{\prime}$ como no exemplo 3.10(c). Como $S$ e $h$ são subgrupos normais em $G$ segue, do teorema anterior, que $E(G, S)=\epsilon(G, S)=\epsilon(G / S)=\epsilon(\mathbb{Z})=2$. Analogamente $E\left(G, K^{\prime}\right)=2$.

4.27 Proposição: Se a aplicação restriçâo $r \epsilon s_{S}^{G}: H^{1}\left(G ; \mathbb{Z}_{2}(G / S)\right) \rightarrow$ $H^{1}\left(S ; \mathbb{Z}_{2}(G / S)\right)$ é trivial $\epsilon[G: S]=\infty$ entâo

$$
E(G, S)=\epsilon(G, S) .
$$

Demonstração: Seja $\varphi^{*}$ a aplicaçào dada em 4.25. Se $r \epsilon s_{S}^{G}=0$ então her $r \epsilon s_{S}^{G}=H^{1}\left(G ; \mathbb{Z}_{2}(G / S)\right) \supset \operatorname{Ker} \varphi^{*}$ e dai $\epsilon(G, S) \leq E(G, S)$ (por 4.25).

Pelo teorema anterior, $E(G, S) \leq \epsilon(G, S)$. Logo obtemos a igualdade.

4.28 Corolário: Stjam $G$ um grupo $\epsilon S$ um subgrupo $d \epsilon G$ com $[G: S]=\propto$. Se $S$ é finito de ordem impar entào $E(G . S)=\epsilon(G, S)$.

Demonstração: Se $|S|$ é impar entào $|S|$ é invertível en $\mathbb{Z}_{2}(G / S)$. Daí. por [8, III.10.2], $H^{1}\left(S: \mathbb{Z}_{2}(G / S)\right)=0$.

Logo $r \epsilon s_{S}^{G}$ é trivial e portanto o result ado segue da proposição anterior.

4.29 Observação: Nào sabemos se o resultado acima é verdadeiro para $|S|$ par. 
A seguir apresentamos uma outra forma (usando derivaçōes) de ver que $E(G, S)=\epsilon(G, S)$ se $S$ é subgrupo normal em $G$.

4.30 Proposição: Sejam $G$ um grupo e $S$ um subgrupo de $G$ de índice infinito. Se $S$ é um subgrupo normal em $G$ então $e(G, S)=E(G, S)$.

Demonstração: Como $S$ é normal em $G$ e $[G: S]=\infty$ temos que $G / S \dot{e}$ um grupo infinito. Dai, por 4.15(iv) e 4.10 temos que

$$
\begin{aligned}
\epsilon(G, S) & =\epsilon(G / S)=1+\operatorname{dim} H^{1}\left(G / S ; \mathbb{Z}_{2}(G / S)\right) \\
& =1+\operatorname{dim} \frac{\operatorname{Dcr}\left(G / S, \mathbb{Z}_{2}(G / S)\right)}{P\left(G / S, \mathbb{Z}_{2}(G / S)\right)} .
\end{aligned}
$$

Logo para provar a proposiçào é suficiente, pelo lema 2.5 , mostrar que os grupos $A=\frac{\operatorname{Der}\left(G / S, \mathbb{Z}_{2}(G / S)\right)}{P\left(G / S, \mathbb{Z}_{2}(G / S)\right)}$ e $B=\frac{\operatorname{Der}\left(G, S, \mathbb{Z}_{2}(G / S)\right)}{P\left(G, S, \mathbb{Z}_{2}(G / S)\right)}$ são isomorfos.

Seja $\psi: A \rightarrow B$ definida por

$\psi\left(f+P\left(G / S, \mathbb{Z}_{2}(G / S)\right)\right)=\hat{f}+P\left(G, S, \mathbb{Z}_{2}(G / S)\right)$, onde $\hat{f}(g):=f(\bar{g})$.

Claramente $\hat{f}$ é uma derivaçào (visto que $f$ é uma derivação) e $\tilde{f_{\mid S}}=0$ pois $\tilde{f}(s)=f(\bar{s})=f(1)=0$.

Além disso, se $f \in P\left(G / S, \mathbb{Z}_{2}(G / S)\right)$ entào $f=d_{m}$ para algum $m=\overline{g_{1}}+\ldots+\bar{g}_{t} \in \mathbb{Z}_{2}(G / S)$ dai como $\bar{g} m=g m$, temos que $\hat{f}(g)=f(\bar{g})=d_{m}(\bar{g})=$ $\bar{g} m-m=g m-m=d_{m}(g)$, isto é, $\hat{f} \in P\left(G, S, \mathbb{Z}_{2}(G / S)\right)$.

భ' é um homomorfismo pois $(f \dot{+} h)(g):=(f+h)(\bar{g})=f(\bar{g})+h(\bar{g})=$ $\hat{f}(g)+\hat{h}(g)=(\hat{f}+\hat{h})(g)$ para todo $g \in G$.

Agora observemos que dado $\dot{f} \in \operatorname{Der}\left(G, S, \mathbb{Z}_{2}(G / S)\right)$ podemos definir uma derivação $f: G / S \rightarrow \mathbb{Z}_{2}(G / S)$ por $f(\bar{g}):=\hat{f}(g)$. Temos que $f$ está bem definida pois se $\overline{g_{1}}=\overline{g_{2}}$ então $g_{1}^{-1} g_{2} \in S$. Daj, como $\tilde{f}_{\mid S}=0$ e $\tilde{f}$ é derivaçào temos $0=\tilde{f}\left(g_{1}^{-1} g_{2}\right)=$ $g_{1}^{-1} \hat{f}\left(g_{2}\right)+\hat{f}\left(g_{1}^{-1}\right)$ e portanto $g_{1}^{-1} \dot{f}\left(g_{2}\right)=\hat{f}\left(g_{1}^{-1}\right)=g_{1}^{-1} \hat{f}\left(g_{1}\right)$, de onde segue a igualdade $\tilde{f}\left(g_{2}\right)=\tilde{f}\left(g_{1}\right)$. Temos entào uma aplicaçào bem definida $\varphi: B \rightarrow A$ dada por $\varphi\left(\tilde{f}+P\left(G, S, \mathbb{Z}_{2}(G / S)\right)\right)=f+P\left(G / S, \mathbb{Z}_{2}(G / S)\right)$ onde $f(\bar{g})=\tilde{f}(g)$.

É fácil ver que $\varphi$ é uma inversa para $\psi$ e portanto obtemos o isomorfismo desejado. 
Finalizandò esta seç̧ào nós observamos que o invariante $E(G, S, M)$ foi definido somente quando $[G: S]=\infty$, mas é interessante observar ( compare com o lema 2.5), o seguinte fato:

4.31 Lema: $S \epsilon[G: S]<\infty$ entâo $\frac{D \epsilon r\left(G, S, \mathbf{Z}_{2}(G / S)\right)}{P\left(G, S, \mathbf{Z}_{2}(G / S)\right)}=0$.

Demonstração: Sabemos que se $[G: S]<\infty$ entâo $\epsilon(G, S)=$ $\operatorname{dim} H^{0}(G ; E(G / S))=0$. Logo, da sequência exata (1) (na demonstração de 4.25), obtemos que $\operatorname{Ker} \varphi^{*}=\operatorname{Im} \delta=0$. Agora observe que nào usamos o fato que $[G: S]=\infty$ para obter (2) e (4) na demonstração de 4.24, logo as afirmaçōes (2) e (4) são também válidas para $[G: S]<\infty$. Dai temos,

$\operatorname{dim} \frac{\operatorname{D\epsilon r}\left(G, S, \mathbb{Z}_{2}(G / S)\right)}{P\left(G, S, \mathbb{Z}_{2}(G / S)\right)}=\operatorname{dim} \operatorname{Im} \psi \leq \operatorname{dim} \operatorname{Ker} t=\operatorname{dim} \operatorname{Ker} \varphi^{*}=0$.

\section{Uma interpretação para $E(G, S, M)$ em termos de cohomologia relativa de complexos.}

Sejam $G$ um grupo, $S$ um subgrupo de $G$ e $M$ um $\mathbb{Z}_{2} G$-módulo qualquer. Nesta seç̧ào apresentamos uma interpretação para $E(G, S, M)$ (em particular para $E(G, S)$ ) em termos de cohomologia relativa de complexos, mais precisamente, em termos de pares Eilenberg-Maclane $(X, Y)=K(G, S, 1)$. Dai, o invariante end $e(G, S)$ também terá tal interpretação sempre que a igualdade $e(G, S)=E(G, S)$ for verdadeira.

Para obter esta interpretaçâo faremos uso de um resultado, devido a Bierj e Eckmann, que nos dá, uma interpretaçâo topológica da sequência exata do par grupo $\left(G, S=\left\{S_{i}\right\}_{i \in J}\right)$.

5.1 Proposição: [i, teor.1.3] Stjam $(G, \mathcal{S})$ um par grupo $\in M$ um $\mathbb{Z}_{2} G$-módulo. Se (X.Y) $\epsilon$ um par Eilenberg-MacLane realizando topologicamente $(G, \mathcal{S})$ entâo as sequências exatas de cohomologia do par grupo $(G, S)$ (com coeficientes em $M$ ) 
$\epsilon$ do par $\left(X, Y^{\prime}\right)$ (tomada com cocficientes locais $\mathcal{M}$ ) sâo isomorfas.

Mais precisamente, temos os seguintes diagramas comutativos (a menos de sinal como indicado)

$$
\begin{aligned}
& \cdots \rightarrow H^{k}(G, \mathcal{S} ; M) \rightarrow H^{k}(G ; M) \rightarrow \prod_{i} H^{k}\left(S_{i} ; M\right) \rightarrow H^{k+1}(G, \mathcal{S} ; M) \rightarrow \cdots \\
& \downarrow(-1)^{k+1} \downarrow \quad \downarrow(-1)^{k+1} \downarrow \\
& \cdots \rightarrow H^{k}(X, Y ; \mathcal{M}) \rightarrow H^{k}(X ; \mathcal{M}) \rightarrow \prod_{i} H^{k}\left(Y_{i} ; \mathcal{M}\right) \rightarrow H^{k+1}\left(X, Y_{i} ; \mathcal{M}\right) \rightarrow \cdots
\end{aligned}
$$

onde as aplicaçôes verticais sâo todas isomorfismos.

Nota: Na realidade em [7], os autores apresentam o resultado anterior para $\mathbb{Z} G$-módulos, mas por 1.3(c), tal resultado é igualmente válido para $\mathbb{Z}_{2} G$-módulos.

Vejamos agora a interpretaçào topológica para $E(G, S, M)$.

5.2 Proposição: Sejam $G$ um grupo, $S$ um subgrupo de $G$ com $[G: S]=$ $\infty \in M^{\prime}$ um $\mathbb{Z}_{2} G$-módulo. Se $(X, Y)$ é um um par Eilenberg-MacLane realizando topologicamente $(G, S)$ entâo

$$
E(G, S, M)=1+\operatorname{dim} \frac{H^{1}(X, Y ; \mathcal{M})}{M^{S} / M^{G}}
$$

$\epsilon$ em particular,

$$
E(G, S)=1+\operatorname{dim} \frac{H^{1}\left(X, Y ; \mathbb{Z}_{2}(G / S)\right)}{\left(\mathbb{Z}_{2}(G / S)\right)^{S}},
$$

onde $H^{1}(X . Y ; \mathcal{M}) \in H^{1}\left(X, Y ; \mathbb{Z}_{2}(G / S)\right)$ são tomadas com coeficientes locais dados pelos $\mathbb{Z}_{2} G$-módulos $M \in \mathbb{Z}_{2}(G / S)$, respectivamente.

Demonstração: Pela proposiçâo anterior temos o seguinte diagrama comutativo (a menos de sinal) com linhas exatas e isomorfismos verticais

$$
\begin{aligned}
& 0 \rightarrow H^{0}(G ; M) \rightarrow H^{0}(S ; M) \stackrel{\mathcal{\varepsilon}}{\rightarrow} H^{1}(G, S ; M) \stackrel{\lrcorner}{\rightarrow} H^{1}(G ; M) \stackrel{\text { res⿱宀 } \stackrel{G}{\rightarrow}}{\rightarrow} H^{1}(S ; M) \rightarrow \cdots \\
& \downarrow \simeq \quad \downarrow \simeq(-1) \quad \downarrow \simeq \quad \downarrow \simeq \quad \downarrow \simeq \\
& 0 \rightarrow H^{0}(X ; \mathcal{M}) \rightarrow H^{0}(Y ; \mathcal{M}) \stackrel{\stackrel{\xi^{\prime}}{\rightarrow}}{H^{1}}(X, Y ; \mathcal{M}) \stackrel{J^{\prime}}{\rightarrow} H^{1}(X ; \mathcal{M}) \stackrel{\ulcorner}{\rightarrow} H^{1}(Y ; \mathcal{M}) \rightarrow \cdots
\end{aligned}
$$


Dai Ker $J^{\prime}=\operatorname{Im} \delta^{\prime} \simeq \operatorname{lm} \delta \simeq M^{S} / M^{G}$ e portanto,

$$
\text { Ker } r \in s_{S, M}^{G} \simeq \operatorname{Ker} r=\operatorname{Im} J^{\prime} \simeq \frac{H^{1}(X, Y ; \mathcal{M})}{M^{S} / M^{G}}
$$

onde identificamos Ker $J^{\prime} \operatorname{com} M^{S} / M^{G}$ via o isomorfismo anterior.

Logo $E(G, S, M)=1+\operatorname{dim} \operatorname{Ker} r s_{S, M}^{G}=1+\operatorname{dim} \frac{H^{1}(X, Y ; \mathcal{M})}{M^{S} / M^{G}}$.

5.3 Corolário: Sejam $G$ um grupo, $S$ um subgrupo de $G$ com $[G: S]=\infty$. Se $(X, Y)$ é un par Eilenberg-MacLane realizando topologicamente $(G, S) \epsilon$ $\epsilon(G, S)=E(G, S)$ (por excmplo no caso em que $S$ é normal em $G$ ) então

$$
\epsilon(G, S)=1+\operatorname{dim} \frac{H^{1}\left(X, Y ; \mathbf{Z}_{2}(G / S)\right)}{\left(\mathbf{Z}_{2}(G / S)\right)^{S}}
$$

onde $H^{1}\left(X, Y ; \mathbb{Z}_{2}(G / S)\right)$ é tomada com coeficientes locais.

Demonstração: É consequência imediata da proposição anterior. $O$ caso $S$ normal em $G$ segue de 4.24 .

\subsection{Exemplos:}

(1) Sejam $G=\mathbb{Z} \oplus \mathbb{Z}$ e $S=\{1\} \oplus \mathbb{Z}$. O par $(G, S)$ é realizado topologjcamente por $\left(X, Y^{\prime}\right)=\left(S^{1} \times S^{1}, S^{1} \times\{1\}\right) \equiv\left(T^{2}, S^{1}\right)$. Logo, para todo $\mathbb{Z}_{2} G$-módulo $M$, temos

$$
E(G, S, M)=1+\operatorname{dim} \frac{H^{1}\left(T^{2}, S^{1}, \mathcal{M}\right)}{M^{S} / M^{G}} .
$$

Em particular, como $S$ é normal em $G$, temos por 4.24 , que

$$
\epsilon(G, S)=E(G, S)=1+\operatorname{dim} \frac{H^{1}\left(T^{2}, S^{1} ; \mathbb{Z}_{2}(G / S)\right)}{\left(\mathbb{Z}_{2}(G / S)\right)^{S}}=2 .
$$

(2) Consideremos o grupo $G=\langle a\rangle *\langle b\rangle \simeq \mathbb{Z} * \mathbb{Z}$ e os subgrupos $S_{1}=\left\langle a b a^{-1} b^{-1}\right\rangle$ e $S_{2}=\langle b\rangle$. Seja $X$ um toro $T^{2}$ com disco aberto remorido. Temos que $\left(G, S_{1}\right)$ e $\left(G, S_{2}\right)$ são realizados topologicamente, respectivamente, pelos pares Eilenberg-MacLane $\left(X, Y_{1}=\partial X\right)$ (ver 3.7 - figura) e $\left(X, Y_{2}\right)$ onde e $Y_{2}$ é o subcomplexo, representado pelo gerador $b$ de $\Pi_{1}\left(T^{2}\right)$. Logo, pela proposição anterior, se $M$ é um $\mathbb{Z}_{2} G$-módulo, 
$E\left(G, S_{1}, M\right)=1+\operatorname{dim} \frac{H^{1}\left(X^{\prime}, Y_{1} ; \mathcal{M}\right)}{M^{S_{1}} / M^{G}}$ e $E\left(G, S_{2}, M\right)=1+\operatorname{dim} \frac{H^{1}\left(X, Y_{2} ; \mathcal{M}\right)}{M^{S_{2}} / M^{G}}$

Em particular, levando em consideraçào 4.21 e 3.8 , temos

$$
\begin{aligned}
& E\left(G, S_{1}\right)=1+\operatorname{dim} \frac{H^{1}\left(X, Y_{1} ; \mathbf{Z}_{2}\left(G / S_{1}\right)\right)}{\left(\mathbf{Z}_{2}\left(G / S_{1}\right)^{S_{1}}\right.}=1<e\left(G, S_{1}\right)=\infty \quad \text { e } \\
& E\left(G, S_{2}\right)=1+\operatorname{dim} \frac{H^{1}\left(X, Y_{2} ; \mathbf{Z}_{2}\left(G / S_{2}\right)\right.}{\left(\mathbf{Z}_{2}\left(G / S_{2}\right)\right)^{S_{2}}}=\infty=e\left(G, S_{2}\right) .
\end{aligned}
$$

(3) Sejam $G=\mathbb{Z} \oplus \mathbf{Z}_{2}$ e $S=\{1\} \oplus \mathbf{Z}_{2}$. O par $(G, S)$ é realizado topologicamente por $(X, Y)=\left(S^{1} \times \mathbb{R} P(\infty),\{1\} \times \mathbb{R} P(\infty)\right)$. Dai

$$
E(G, S, M)=1+\operatorname{dim} \frac{H^{1}\left(S^{1} \times \mathbb{R P}(\infty), \mathbb{R} P(\infty) ; \mathcal{M}\right)}{M^{S} / M^{G}},
$$

para todo $\mathbb{Z}_{2} G$-módulo $M$, e como $S$ é normal em $G$,

$$
\epsilon(G, S)=E(G, S)=1+\operatorname{dim} \frac{H^{1}\left(S^{1} \times \mathbb{R} P(\infty), \mathbb{R} P(\infty) ; \mathbb{Z}_{2}(G / S)\right)}{\mathbb{Z}_{2}(G / S)^{S}}=2 .
$$

(4) Sejam $G$ um grupo infinito e $S=\{1\}$ o subgrupo trivial. Considere $X$ um complexo $K(G, 1)$, e o vértice $v_{0} \in X$. 0 par $\left(X, v_{0}\right)$ é um par Eilenberg-MacLane realizando $(G, S)$. Logo, se $M$ é um $\mathbb{Z}_{2} G$-módulo,

$$
E(G,\{1\}, M)=1+\operatorname{dim} \frac{H^{\prime}\left(X, v_{0}, \mathcal{M}\right)}{M / M^{G}} .
$$

En particular,

$$
\epsilon(G)=1+\operatorname{dim} \frac{H^{1}\left(X^{\prime}, v_{0} ; \mathbb{Z}_{2} G\right)}{\mathbb{Z}_{2} G}
$$




\section{CAPÍTULO III}

\section{O Invariante $\tilde{\mathbf{E}}(\mathbf{G}, \mathbf{S})$}

Neste capitulo dados $(G, S)$ com $[G: S]=\infty$, estudamos o invariante $E(G, S, M)$ para o $\mathbb{Z}_{2} G$-módulo particular $M=\mathcal{F}_{S} G$ definido abaixo. Por simplicidade $E\left(G, S, \mathcal{F}_{S} G\right)$ será denotado por $\hat{E}(G, S)$. Na seç̧ào 1 provamos (uma vez que não encontramos a justificativa deste fato) que $\mathcal{F}_{S} G$ é isomorfo ao módulo induzido Ind $d_{S} \overline{Z_{2} S}=\mathbb{Z}_{2} G \otimes S \overline{\mathbb{Z}_{2} S}$. Isto é interessante pois em alguns casos é conveniente usar $\mathcal{F}_{S} G$ e em outros $\operatorname{Ind} d_{S}^{G} \overline{\mathbb{Z}_{2} S}$. Também estendemos a definiçâo de $\tilde{E}(G, S)$ de modo a incluir o caso em que $[G: S]<\infty$ e verificamos que, considerando esta definiçâo, tem-se: $\quad \tilde{E}(G, S)=0 \Longleftrightarrow[G: S]<\infty$. Na seç̧ào 2 apresentamos várias propriedades de $\dot{E}(G, S)$. Na seç̧ào 3 analisamos a relaçâo entre $\hat{E}(G, S)$ e o invariante end $\tilde{\epsilon}(G, S)$ definido por Kropholler e Roller [22].

Como veremos (observaçào 3.6$), \quad \hat{E}(G, S)$ tem certa vantagem em relaçâo a $\hat{\epsilon}(G, S)$ pois $\bar{\epsilon}(G, S)$ fornece alguma informaşâo a respeito de $S$ somente quando $\epsilon(G)=1$ uma vez que, se $\epsilon(G)=\infty$ entâo $\hat{\epsilon}(G, S)=\infty$ para todo subgrupo $S$ de $G$. No entanto para $\dot{E}$ temos exemplos em que $\epsilon(G)=\infty$ e $\dot{E}(G, S) \neq \hat{E}(G, T)$ para subgrupos distintos $S$ e $T$ de $G$, o que nos mostra que $\hat{E}(G, S)$ nos dá informaçào a respeito de $S$ também no caso em que $\epsilon(G)=\propto$. Além disto, $\hat{E}(G, S)$ nos dá uma condição necessária para que $(G, S)$ seja um $D^{n}$-par. o que não ocorre com $\hat{\epsilon}(G, S)$.

\section{O Invariante $\tilde{E}(G, S)$}

Sejam $G$ um grupo, $S$ um subgrupo de $G$ e 


$$
\mathcal{F}_{S} G:=\{B \subset G \mid B \subset F . S \text { para algum subconjunto finito } F \text { de } G\} \text {. }
$$

Claramente $\mathcal{F}_{S} G$ é um $\mathbf{Z}_{2} G$-submódulo de $P G$ com as operaçōes induzidas. Consideremos o $\mathbb{Z}_{2} G$-módulo $I n d G \overline{\mathbb{Z}_{2} S}=\mathbf{Z}_{2} G \otimes_{S} \overline{\mathbf{Z}_{2} S}$ com a $G$-ação natural de módulo induzido $\left(g \cdot\left(g_{1} \otimes m\right)=g g_{1} \otimes m\right)$.

Veremos, no lema abaixo, que é natural considerar Ind $G \overline{\mathbb{Z}_{2} S}$ como um submódulo de $\overline{\mathbb{Z}_{2} G}$, e que este submódulo pode ser identificado, via o isomorfismo $\rho: \overline{\mathbb{Z}_{2} G} \rightarrow P G \quad$ (ver em 1.3.15), com o submódulo $\mathcal{F}_{S} G$ de $P G$.

\subsection{Lema: $O s$ módulos $\mathbb{Z}_{2} G \otimes_{S} \overline{\mathbb{Z}_{2} S}$ e $\mathcal{F}_{S} G$ sâo $\mathbf{Z}_{2} G$-isomorfos.}

Demonstração: Consideremos as aplicaçōes $\mathbf{Z}_{2} G \otimes_{S} \overline{\mathbb{Z}_{2} S}=\operatorname{Ind} d_{S}^{G} \operatorname{Coind}_{\{1\}}^{S} \mathbb{Z}_{2} \stackrel{\varphi}{\rightarrow} \operatorname{Coind}_{S}^{G} \operatorname{Coind}_{\{1\}}^{S} \mathbb{Z}_{2} \stackrel{⿱ ⺌}{\rightarrow} \operatorname{Coind}_{\{1\}}^{G} \mathbb{Z}_{2}=\overline{\mathbb{Z}_{2} G} \stackrel{\rho}{\rightarrow} P G$, onde $\rho$ é o $\mathbb{Z}_{2} G$-isomorfismo dado em 1.3.15, e $\varphi$ e $\psi$ sào as $\mathbb{Z}_{2} G$-aplicaçôes dadas em I.3.8, isto é, se $g_{0} \otimes h \in \mathbb{Z}_{2} G \odot_{S} \operatorname{Hom}\left(\mathbb{Z}_{2} S, \mathbb{Z}_{2}\right)=\mathbb{Z}_{2} G \Theta_{s} \overline{\mathbb{Z}_{2} S}$, então

$$
\varphi\left(g_{0} \otimes h\right)(g)= \begin{cases}\left(g g_{0}\right) \cdot h & \text { se } g g_{0} \in S \\ 0 & \text { caso contrário, }\end{cases}
$$

e se $f \in \operatorname{Hom}_{S}\left(\mathbb{Z}_{2} G, \operatorname{Hom}\left(\mathbb{Z}_{2} S, \mathbb{Z}_{2}\right)\right), \psi^{\prime}(f)(g)=f(g)(1)$, onde $1 \in \mathbb{Z}_{2} S$.

Como $\varphi$ é $\mathbb{Z}_{2} G$-monomorfismo e $\psi$ é $\mathbb{Z}_{2} G$-isomorfismo, podemos ver $\mathbb{Z}_{2} G \otimes S \overline{\mathbb{Z}_{2} S}$ como um $\mathbb{Z}_{2} G$-submódulo de $\overline{\mathbb{Z}_{2} G}$, que será $\mathbb{Z}_{2} G$-isomorfo (via $\rho$ ) ao $\mathbb{Z}_{2} G$ submódulo $\rho\left((\psi \circ \varphi)\left(\mathbb{Z}_{2} G \otimes s \overline{\mathbb{Z}_{2} S}\right)\right)$ de $P G$.

Logo para concluir o lema, basta provar o seguinte fato:

Afirmaşão: $\left.\rho(\dot{\psi} \circ \circ)\left(\mathbb{Z}_{2} G \Theta_{S} \overline{\mathbb{Z}_{2} S}\right)\right)=\mathcal{F}_{S} G$.

Isto segue das seguintes observaçoes:

(i) Um elemento $B \in \mathcal{F}_{S} G \Leftrightarrow B=g_{0} S_{0} \cup \ldots \cup g_{k} S_{k}$, com $g_{i} \in G, S_{i} \subset S$ e $k \in N$.

(ii) Da mesma forma que temos o $\mathbb{Z}_{2} G$-isomorfismo $\rho: \overline{\mathbb{Z}_{2} G} \rightarrow P G$, podemos também definir o $\mathbb{Z}_{2} S$-isomorfismo $\hat{\rho}: \overline{\mathbb{Z}_{2} S} \rightarrow P S$. Se considerarmos a injeção natural $\overline{\mathbb{Z}_{2} S} \stackrel{\dot{J}}{\rightarrow} \overline{\mathbb{Z}_{2} G}, \quad h \rightarrow \hat{h}$, onde $\hat{h}$ é definida nos geradores por

$$
\hat{h}(g)= \begin{cases}h(g) & \text { se } g \in S \\ 0 & \text { caso contrário }\end{cases}
$$


então temos o seguinte diagrama comutativo:

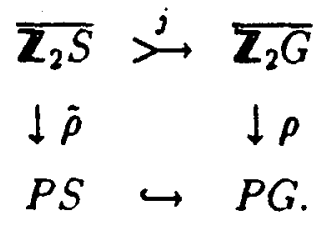

(iii) Se $g_{0} \otimes h_{0}$ é um elemento básico de $\mathbb{Z}_{2} G \otimes s \overline{\mathbf{Z}_{2} S}$ então $(\psi \circ \varphi)\left(g_{0} \otimes h_{0}\right)\left(g^{-1}\right)= \begin{cases}{\left[\left(g^{-1} g_{0}\right) \cdot h_{0}\right](1)=h_{0}\left(g^{-1} g_{0}\right)} & \text { se } g^{-1} g_{0} \in S \\ 0 & \text { caso contrário. }\end{cases}$

Daí

$$
\begin{aligned}
\rho\left((\psi \circ \varphi)\left(g_{0} \otimes h_{0}\right)\right) & =\left\{g \in G \mid(\psi \circ \varphi)\left(g_{0} \otimes h_{0}\right)\left(g^{-1}\right)=1\right\} \\
& =\left\{g \in G \mid g^{-1} g_{0} \in S \text { e } h_{0}\left(g^{-1} g_{0}\right)=1\right\} \\
& =\left\{g \in G \mid g^{-1} g_{0} \in S \text { e } h_{0}\left(\left(g_{0}^{-1} g\right)^{-1}\right)=1\right\} \\
& =\left\{g \in G \mid g_{0}^{-1} g \in \dot{\rho}\left(h_{0}\right)\right\} \\
& =\left\{g \in G \mid g_{0}^{-1} g=s \in \dot{\rho}\left(h_{0}\right)\right\} \\
& =\left\{g_{0} . s \mid s \in \dot{\rho}\left(h_{0}\right)\right\},
\end{aligned}
$$

isto é, $\rho\left((\psi \circ \varphi)\left(g_{0} \otimes h_{0}\right)\right)=g_{0} . S_{0} \in \mathcal{F}_{S} G$, onde $S_{0}=\tilde{\rho}\left(h_{0}\right) \subset S$.

Agora se $u=g_{0} \otimes h_{0}+g_{1} \otimes h_{1} \in \mathbb{Z}_{2} G \otimes s \overline{\mathbb{Z}_{2} S}$ então

$$
\begin{aligned}
& \rho(\psi \circ \varphi)(u)=\left\{g \in G \mid \rho\left(\psi^{\prime} \circ \varphi\right)\left(g_{0} \odot h_{0}+g_{1} \otimes h_{1}\right)\left(g^{-1}\right)=1\right\} \\
&=\left\{g \in G \mid \rho\left(\psi^{\prime} \circ \varphi\right)\left(g_{0} \odot h_{0}\right)\left(g^{-1}\right)+\rho\left(\psi^{\prime} \circ \varphi\right)\left(g_{1} \otimes h_{1}\right)\left(g^{-1}\right)=1\right\} \\
&=\left\{\begin{array}{l}
\left.\rho \in G \mid \begin{array}{l}
\rho\left(\psi^{\prime} \circ \varphi\right)\left(g_{0} \odot h_{0}\right)\left(g^{-1}\right)=1 \text { ou (exclusivo) } \\
\rho(\psi \circ \varphi)\left(g_{1} \odot h_{1}\right)\left(g^{-1}\right)=1
\end{array}\right\} \\
=
\end{array}\right. \\
& g_{0} . S_{0} \cup g_{1} \cdot S_{1} \in \mathcal{F}_{S} G, \text { onde } S_{i}=\dot{\rho}\left(h_{i}\right) \subset S, i=0,1 .
\end{aligned}
$$

Mais geralmente, se $u=g_{0} \otimes h_{0}+g_{1} \otimes h_{1}+\cdots+g_{k} \otimes h_{k} \in \mathbb{Z}_{2} G \otimes s \overline{\mathbb{Z}_{2} S}$, então $\rho(\psi \circ \varphi)(u)=g_{0} . S_{0} \cup \ldots \cup g_{k} . S_{k}, \operatorname{com} S_{i}=\dot{\rho}\left(h_{i}\right), i=0,1, \ldots, k$.

(iv) Se $B=g_{0} . S_{0} \cup \ldots \cup g_{k} . S_{k}$ é um elemento qualquer de $\mathcal{F}_{S} G$, então é fácil ver que

$$
B=\rho(\psi \circ \varphi)\left(g_{0} \otimes \hat{\rho}^{-1}\left(S_{0}\right)+\cdots+g_{k} \otimes \hat{\rho}^{-1}\left(S_{k}\right)\right) \in \rho\left((\psi \circ \varphi)\left(\mathbb{Z}_{2} G \otimes \otimes_{S} \overline{\mathbb{Z}_{2} S}\right)\right) .
$$




\section{O invariante $\hat{\mathbf{E}}(\mathbf{G}, \mathbf{S})$ :}

Sejam $G$ um grupo e $S$ um subgrupo de $G$ com $[G: S]=\infty$. Se $M=$ $\mathcal{F}_{S} G \simeq \mathbf{Z}_{2} G \otimes s \overline{\mathbf{Z}_{2} S} \simeq \operatorname{Ind} d_{S}^{G} P S$ entào denotaremos $E(G, S, M)$ por $\dot{E}(G, S)$. Isto é,

$$
\hat{E}(G, S)=1+\operatorname{dim} \operatorname{Ker} \operatorname{res}_{S}^{G},
$$

onde $r \epsilon s_{S}^{G}: H^{1}\left(G ; \mathcal{F}_{S} G\right) \rightarrow H^{1}\left(S ; \mathcal{F}_{S} G\right)$ é a aplicação restrição.

1.2 Lema: $\tilde{E}(G, S)$ é um invariante algébrico para pares de grupos $(G, S)$ $\operatorname{com}[G: S]=\infty$.

Demonstração: Seja $\alpha:(G, S) \rightarrow(L, K)$ um isomorfismo de pares de grupos. Vamos provar que existe $\phi: \mathcal{F}_{K} L \rightarrow \mathcal{F}_{S} G$, isomorfismo de grupos abelianos, tal que $\phi(\alpha(g) . u)=g . \phi(u)$, para $g \in G$ e $u \in \mathcal{F}_{k} L$. Dai $\left((G, S), \mathcal{F}_{S} G\right)$ e $\left((L, K), \mathcal{F}_{K} L\right)$ são isomorfos na categotegoria $\mathcal{C}$, definida em II.2, e então, por II.2.3 teremos

$$
\tilde{E}(G, S)=E\left(G, S, \mathcal{F}_{S} G^{\prime}\right)=E\left(L, K, \mathcal{F}_{K} L\right)=\hat{E}\left(L, K^{\prime}\right)
$$

Primeiro observemos que o isomorfismo $\alpha_{\mid}: S \rightarrow K$ induz um isomorfismo $\gamma: \overline{Z_{2} K^{\prime}} \rightarrow \overline{Z_{2} S}$, onde nos geradores, $\gamma(f)$ é dado por $\gamma(f)(s)=f(\alpha(s))$ e é estendido por linearidade.

Agora, considere a aplicaçào $\phi: \mathbb{Z}_{2} L \Theta_{K} \overline{\mathbb{Z}_{2} K^{\prime}} \rightarrow \mathbb{Z}_{2} G \otimes_{S} \overline{\mathbb{Z}_{2} S}$, definida nos geradores por $\phi(l \odot f)=a^{-1}(l) \odot \gamma(f)$ (e estendida por linearidade). Claramente $\phi$ é um isomorfismo de grupos abelianos. Além disso,

$$
\begin{aligned}
\phi(a(g) .(l \otimes f)) & =\phi(\alpha(g) l \otimes f)=a^{-1}(a(g) l) \otimes \gamma(f) \\
& =g a^{-1}(l) \otimes \gamma(f)=g \cdot\left(\alpha^{-1}(l) \otimes \gamma(f)\right) \\
& =g \cdot \phi(l \otimes f) .
\end{aligned}
$$

Logo, levando em conta 1.1, tem-se o resultado desejado.

Nota: De acordo com o lema 2.5 do capítulo anterior, para $M=\mathcal{F}_{S} G$,

$$
\text { Ker } r \epsilon s_{S}^{G} \simeq \frac{D \epsilon r\left(G, S, \mathcal{F}_{S} G\right)}{P\left(G, S, \mathcal{F}_{S} G\right.},
$$


e, se $[G: S]=\infty$, entào $\dot{E}(G, S)$ tem a seguinte interpretação:

$$
\dot{E}(G, S)=1+\operatorname{dim} \frac{\operatorname{Der}\left(G, S, \mathcal{F}_{S} G\right)}{P\left(G, S, \mathcal{F}_{S} G\right)} .
$$

$\dot{E}$ interessante (de modo similar à ll.4.31) analisar o quociente $\frac{\operatorname{Der}\left(G, S, \mathcal{F}_{S} G\right)}{P\left(G, S, \mathcal{F}_{S} G\right)}$ no caso em que $[G: S]<\infty$.

1.3 Lema: $S \epsilon[G: S]<\infty \operatorname{cntäo} \frac{D \operatorname{cr}\left(G, S, \mathcal{F}_{S} G\right)}{P\left(G, S, \mathcal{F}_{S} G\right)}=0$.

Demonstraşão: Se $[G: S]<\infty$ entào $\mathcal{F}_{S} G=P G$. Mas $H^{1}(G ; P G)=0$ por 1.3.16. Logo temos

$$
\frac{\operatorname{Der}\left(G, S, \mathcal{F}_{S} G\right)}{P\left(G, S, \mathcal{F}_{S} G\right)} \simeq \operatorname{Ker} r \varepsilon_{S}^{G} \subset H^{1}\left(G ; \mathcal{F}_{S} G\right)=H^{1}(G ; P G)=0
$$

A proposição seguinte nos dá uma forma de estender a definição de $E(G, S)$ de modo a abranger o caso em que $[G: S]<\infty$ (Note que para $E(G, S)$ nâo conseguimos obter isto). Para demonstrar tal proposiçâo precisamos do resultado abaixo, afirmado em $[20$, p. 54$]$.

1.4 Lema: Seja $\mathcal{A}_{S} G=\left\{A \subset G \mid A+g A \in \mathcal{F}_{S} G, \forall g \in G\right\}$. Então a aplicaçâo

$$
\begin{aligned}
\beta: \mathcal{A}_{S} G & \rightarrow \operatorname{Der}\left(G, \mathcal{F}_{S}(i)\right. \\
B & \rightarrow \beta(B), \quad \text { onde } \quad \beta(B)(g):=B+g B, \forall g \in G,
\end{aligned}
$$

é sobrejetora, isto é, $\operatorname{Der}\left(G, \mathcal{F}_{S} G\right)=P\left(G, \mathcal{A}_{S} G^{\prime}\right)$.

Demonstração: Como $\frac{D \epsilon r(G, P G)}{P(G, P G)}=H^{1}(G ; P G)=0$ e $\mathcal{F}_{S} G \subset P G$, temos que $\operatorname{Der}\left(G, \mathcal{F}_{S} G\right) \subset \operatorname{Der}(G, P G)=P(G, P G)$.

Dai, se $f \in D \in r\left(G, \mathcal{F}_{S} G\right)$ entào $f \in P(G, P G)$; logo existe $B \in P G$ tal que $f=d_{B}$, isto é, $f(g)=B+g B, \forall g \in G$, onde $B \in P G$. Mas do fato que $f(g) \in \mathcal{F}_{S} G$ para todo $g$ (pois $f \in \operatorname{Der}\left(G, \mathcal{F}_{S} G\right.$ )), segue que $B+g B \in \mathcal{F}_{S} G, \forall g \in G$, isto é, $B \in \mathcal{A}_{S} G$.

Portanto $f=\beta(B) \in P\left(G, \mathcal{A}_{S} G\right)=\operatorname{lm} \beta$. 
1.5 Proposição: $S e(G, S)$ é um par de grupos satisfazendo $[G: S]=\infty$, então a sequência

$$
0 \rightarrow\{\emptyset, G\} \stackrel{a}{\rightarrow} \frac{\mathcal{A}_{S} G \cap(P G)^{S}}{\mathcal{F}_{S} G \cap(P G)^{S}} \stackrel{\dot{\beta}}{\rightarrow} \frac{D \epsilon r\left(G, S, \mathcal{F}_{S} G\right)}{P\left(G, S, \mathcal{F}_{S} G\right)} \rightarrow 0,
$$

com aplicaçôes $\alpha$ e $\hat{\beta}$ dadas por: $\alpha(A):=[A]=A+\mathcal{F}_{S} G \cap(P G)^{S}$ e $\hat{\beta}([B]):=\beta(B)+$ $P\left(G, S, \mathcal{F}_{S} G\right)$, onde $\beta$ é como no lema anterior, é exata.

Demonstração: A aplicaçào $\alpha$ é injetora pois, $\alpha(G)=0 \Rightarrow$ $G \in \mathcal{F}_{S} G \cap(P G)^{S} \Rightarrow G \subset F . S$ para algum subconjunto finito $F$ de $G$. Dai $[G: S]<\infty$, o que é uma contradição.

Vejamos que Ker $\dot{\beta}=\operatorname{lm} a=\{[\emptyset],[G]\}$.

Claramente $\operatorname{Im} \alpha \subset \operatorname{lier} \hat{\beta}$ pois, para todo $g \in G, \beta(\theta)(g)=0$ e $\beta(G)(g)=G+g G=G+G=0$ e dai $\hat{\beta}([\phi])=\tilde{\beta}([G])=0$. Agora, se $[B] \epsilon$ $\frac{\mathcal{A}_{S} G \cap(P G)^{S}}{\mathcal{F}_{S} G \cap(P G)^{S}}$ é um elemento de $\operatorname{Ker} \hat{\beta}$ entào $\beta(B) \in P\left(G, S, \mathcal{F}_{S} G\right)$. Logo existe $A \in$ $\mathcal{F}_{S} G$ satisfazendo $\beta(B)=d_{A}$, isto é, $B+g B=A+g A$, para todo $g \in G$. Daí $B+A=g(B+A)$, para todo $g \in G$, ou seja, $B+A \in(P G)^{G}=\{\emptyset, G\}$ e portanto $[B]=[0]=\alpha(\theta)$ (se $B+A=\emptyset)$ ou $[B]=[G]=a(G)$ (se $B+A=G)$.

Finalmente, para ver que $\hat{\beta}$ é sobrejetora basta usar o lema anterior.

1.6 Corolário: $S \epsilon[G: S]=\infty$, entâo

$$
\tilde{E}(G, S)=\operatorname{dim} \frac{\mathcal{A}_{S} G \cap(P G)^{S}}{\mathcal{F}_{S} G \cap(P G)^{S}}=\operatorname{dim} \frac{\left(\mathcal{A}_{S} G\right)^{S}}{\left(\mathcal{F}_{S} G\right)^{S}} .
$$

Demonstração: Se $[G: S]=\propto$ então, pela proposição anterior,

$$
\operatorname{dim} \frac{\mathcal{A}_{S} G \cap(P G)^{S}}{\mathcal{F}_{S} G \cap(P G)^{S}}=1+\operatorname{dim} \frac{D c r\left(G, S, \mathcal{F}_{S} G\right)}{P\left(G, S, \mathcal{F}_{S} G\right)}=\tilde{E}(G, S)
$$

Podemos entâo definir, para qualquer par de grupos $(G, S) \operatorname{com}[G: S]$ não necessariamente infinito,

$$
\tilde{E}(G, S)=\operatorname{dim} \frac{\mathcal{A}_{S} G \cap\left(P G_{i}\right)^{S}}{\mathcal{F}_{S} G \cap\left(P(i)^{S}\right.}
$$


Nesta condiçòes temos:

1.7 Lema: $\dot{E}(G, S)=0$ se, $\epsilon$ somente sc, $[G: S]<\infty$.

Demonstração: $\Leftrightarrow$ Suponhamos por absurdo que $[G: S]=\infty$. Então (por 1.5 ou definição inicial de $\hat{E}(G, S)$ ) tem-se $\tilde{E}(G, S) \geq 1$, o que contradiz a hipótese. Logo $[G: S]<\infty$.

$(\Leftrightarrow)$ Se $[G: S]<\infty$ entào $\mathcal{F}_{S} G=P G=\mathcal{A}_{S} G$. Daí $\hat{E}(G, S)=0$.

1.8 Lema: $\hat{E}(G,\{1\})=\epsilon(G)$.

Demonstração: Se $S=\{1\}$ entào temos que $(P G)^{S}=P G$, $\mathcal{F}_{S} G=\{A \subset G \mid A \subseteq F, F$ finito de $G\}=F G$, e $\frac{\mathcal{A}_{S} G}{\mathcal{F}_{S} G}=\left(\frac{P G}{F G}\right)^{G}$. Dai $\hat{E}(G,\{1\})=$ $\operatorname{dim}\left(\frac{P G}{F G}\right)^{G}=\epsilon(G)$.

\section{Propriedades.}

Nesta seç̧ào apresentaremos algumas propriedades de $\tilde{E}(G, S)$.

Inicialmente, estudamos a relação entre os dois invariantes introduzido por nós, $E(G, S)$ e $\dot{E}(G, S)$.

2.1 Proposição: $S \epsilon(G, S)$ é um par de grupos com $[G: S]=\infty$ entâo

$$
E(G, S) \leq \hat{E}(G, S)
$$

Demonstração: Consideremos a sequência exata de $\mathbb{Z}_{2} S$-módulos

$$
0 \rightarrow \mathbb{Z}_{2}=\{\emptyset, S\} \rightarrow P S \rightarrow Q=\frac{P S}{\{\emptyset, S\}} \rightarrow 0 .
$$

Como $\mathbb{Z}_{2} G$ é $\mathbb{Z}_{2}$ S-livre obtemos, tensorizando por $\mathbb{Z}_{2} G$, a seguinte sequência exata:

$$
0 \rightarrow \mathbb{Z}_{2} G \otimes S \mathbb{Z}_{2} \stackrel{\circ}{\rightarrow} \mathbb{Z}_{2} G \otimes_{S} P S \stackrel{\pi}{\rightarrow} \mathbb{Z}_{2} G \otimes S Q \rightarrow 0 .
$$

Esta sequência induz a sequência exata longa em cohomologia 


$$
\begin{aligned}
0 \rightarrow H^{0}\left(G ; \operatorname{Ind}_{S}^{G} \mathbb{Z}_{2}\right) \rightarrow H^{0}\left(G ; \operatorname{Ind}_{S}^{G} P S\right) \rightarrow H^{0}\left(G ; \operatorname{Ind}_{S}^{G} Q\right) \rightarrow \\
\quad \rightarrow H^{1}\left(G ; \operatorname{Ind}_{S}^{G} \mathbb{Z}_{2}\right) \stackrel{\phi^{*}}{\rightarrow} H^{1}(G ; \operatorname{Ind} G S S) \rightarrow H_{S}^{1}\left(G ; \operatorname{Ind}_{S}^{G} Q\right) \rightarrow \cdots
\end{aligned}
$$

Uma vez que $[G: S]=\infty$ temos, por 1.3.11, que

$$
H^{0}\left(G ; \ln d_{S}^{G} \mathbf{Z}_{2}\right)=H^{0}\left(G ; \operatorname{In} d_{S}^{G} P S\right)=H^{0}\left(G ; \operatorname{In} d_{S}^{G} Q\right)=0
$$

e portanto $\phi^{*}: H^{1}\left(G ; \operatorname{Ind} d_{S}^{G} \mathbb{Z}_{2}\right) \rightarrow H^{1}\left(G ; \operatorname{Ind} d_{S}^{G} P S\right)$ é um monomorfismo. Dai, aplicando Il.2.7(ii) para $N=\operatorname{Ind} d_{S}^{G} \mathbf{Z}_{2}$ e $M=\operatorname{Ind} G S S$, temos que

$$
E(G, S)=E\left(G, S, \operatorname{In} d_{S}^{G} \mathbb{Z}_{2}\right) \leq E\left(G, S, \operatorname{In} d_{S}^{G} P S\right)=\dot{E}(G, S)
$$

2.2 Corolário: Se $G=\langle a\rangle *\langle b\rangle \simeq \mathbb{Z} * \mathbb{Z} \epsilon S=\langle b\rangle$, entào $\tilde{E}(G, S)=\infty$.

Demonstração: Vimos no exemplo II.3.8, que $E(G, S)=\infty$, logo o resultado segue da desigualdade acima.

A igualdade na proposição anterior nem sempre ocorre como mostra o exemplo abaixo.

2.3 Exemplo: Sejam $G=\mathbb{Z}_{2} * \mathbb{Z}_{2}$ e $S=\mathbb{Z}_{2}$. Entào $E(G, S)=1<$ $\hat{E}(G, S)=2$. De fato, por II.4.15(v), $\epsilon(G, S)=1$. Logo, pelo teorema II.4.24 também $E(G, S)=1$. Agora, que $\hat{E}(G, S)=2$, seguirá de 3.10 (secçào seguinte) e do fato que $\epsilon(G)=2$.

2.4 Observação: Apesar das desigualdades

$$
E(G, S) \leq \epsilon(G, S) \quad \text { e } \quad E(G, S) \leq \hat{E}(G, S)
$$

serem verdadeiras, não existe nenhuma relação entre $\epsilon(G, S)$ e $\tilde{E}(G, S)$ pois, no exemplo acima temos que

$$
\epsilon(G, S)=1<\hat{E}(G, S)=2
$$


Agora, se $(G, S)$ é como no exemplo 11.3 .7 concluiremos por $2.8(\mathrm{i})$ abaixo que $\tilde{E}(G, S)=1$, pois $(G, S)$ é um $P D^{2}$-par. No entanto $\epsilon(G, S)=\infty$ por II.4.15(vi). Logo

$$
\tilde{E}(G, S)=1<e(G, S)=\infty .
$$

2.5 Proposição: Sejam $S$ e $T$ subgrupos de $G$ satisfazendo $S \leq T \leq G$. Se $[G: T]=\infty$ então:

(i) $E\left(G, T, \mathcal{F}_{S} G\right) \leq \dot{E}(G, S) \leq E\left(G, S, \mathcal{F}_{T} G\right)$, e

(ii) $E\left(G, T, \mathcal{F}_{S} G\right) \leq \dot{E}(G, T) \leq E\left(G, S, \mathcal{F}_{T} G\right)$.

Demonstração: Vejamos apenas (i). A afirmação (ii) prova-se de modo análogo.

A desigualdade $E\left(G, T, \mathcal{F}_{S} G^{\prime}\right) \leq \dot{E}(G, S)=E\left(G, S, \mathcal{F}_{S} G\right)$ é obtida de II.2.7(i) tomando $M=\mathcal{F}_{S} G$.

Para obter $\tilde{E}(G, S) \leq E\left(G, S, \mathcal{F}_{T} G\right)$ nós usamos o fato que a inclusão $\mathcal{F}_{S} G \subset \mathcal{F}_{T} G$ induz um mononorfismo $\phi^{*}: H^{1}\left(G ; \mathcal{F}_{S} G\right) \rightarrow H^{1}\left(G ; \mathcal{F}_{T} G\right)$. Para provarmos este fato (que é afirmado em [20,4.2(ii)]), raciocinamos como na proposiçâo anterior.

Consideremos a sequência exata

$$
0 \rightarrow \operatorname{In} d_{S}^{T} \overline{\mathbb{Z}_{2} S} \stackrel{i}{\rightarrow} \overline{\mathbb{Z}_{2} T} \rightarrow Q=\frac{\overline{\mathbb{Z}_{2} T}}{\operatorname{Ind} d_{S}^{T} \overline{\mathbb{Z}_{2} S}} \rightarrow 0,
$$

onde $i$ é a composta $I n d_{S}^{T} \overline{\mathbb{Z}_{2} S} \stackrel{\varphi}{\hookrightarrow} \operatorname{Coind} d_{S}^{T} \overline{\mathbb{Z}_{2} S} \stackrel{\psi}{\rightarrow} \overline{\mathbb{Z}_{2} T}$, e $\varphi$ e $\psi$ sào como em I.3.8.

Visto que $\mathbb{Z}_{2} G$ é livre como $\mathbb{Z}_{2} T$-módulo, Ind $d_{T}^{G}$ é um funtor exato. Se aplicarmos este funtor na sequência acima obtemos

$$
0 \rightarrow \operatorname{In} d_{S}^{G} \overline{\mathbb{Z}_{2} S} \simeq \operatorname{In} d_{T}^{G} \operatorname{In} d_{S}^{T} \overline{\mathbb{Z}_{2} S} \rightarrow \operatorname{In} d_{T}^{G} \overline{\mathbb{Z}_{2} T} \rightarrow \operatorname{In} d_{T}^{G} Q \rightarrow 0 .
$$

Isto induz a sequência exata longa em cohomologia

$$
\begin{array}{r}
0 \rightarrow H^{0}\left(G ; \operatorname{Ind}_{S}^{G} \overline{\mathbb{Z}_{2} S}\right) \rightarrow H^{0}\left(G ; \ln d_{T}^{G} \overline{\mathbb{Z}_{2} T}\right) \rightarrow H^{0}\left(G ; \operatorname{Ind}_{T}^{G} Q\right) \rightarrow \\
\rightarrow H^{1}\left(G ; \operatorname{Ind}_{S}^{G} \overline{\mathbb{Z}_{2} S}\right) \stackrel{\oplus^{*}}{\rightarrow} H^{1}\left(G ; \operatorname{Ind} d_{T}^{G} \overline{\mathbb{Z}_{2} T}\right) \rightarrow \cdots
\end{array}
$$

Como $[G: S]=\infty$, temos que os tres primeiros termos da sequência sâo nulos e dai $\phi^{*}: H^{1}\left(G ; \operatorname{Ind} d_{S}^{G} \overline{\mathbb{Z}_{2} S}\right) \rightarrow H^{1}\left(G ; \operatorname{Ind} d_{T}^{G} \overline{\mathbb{Z}_{2} T}\right)$ é monomorfismo como queríamos. 
Logo, aplicando II.2.7(ii), para $N=\operatorname{In} d_{S}^{G} \overline{\mathbb{Z}_{2} S} \simeq \mathcal{F}_{S} G$ e $M=\operatorname{In} d_{T}^{G} \overline{\mathbb{Z}_{2} T} \simeq$ $\mathcal{F}_{T} G$, obtemos

$$
\dot{E}(G, S)=E\left(G, S, \mathcal{F}_{S} G\right) \leq E\left(G, S, \mathcal{F}_{T} G\right)
$$

\subsection{Corolário:}

(i) Sejam $S \leq T \leq G \operatorname{com}[G: T]=\infty$. Sc $[T: S]<\infty$ então $\tilde{E}(G, T) \leq \tilde{E}(G, S)$.

(ii) Se T' é um subgrupo finitamente gerado de $G,[G: T]=\infty \in \epsilon(T)=1$ entâo $e(G) \leq \hat{E}(G, T)$.

\section{Demonstração:}

(i) Se $[T: S]<\infty$ entâo $\mathcal{F}_{S} G=\mathcal{F}_{T} G$. Dai a desigualdade é consequência imediata da proposiçâo anterior.

(ii) Considerando $S=\{1\}$ na proposiçào anterior, como $\mathcal{F}_{\{1\}} G=F G \simeq$ $\mathbf{Z}_{2} G$ obtemos $E\left(G, T, \mathbf{Z}_{2} G\right) \leq \hat{E}(G, T)$.

Agora, por 1.3 .9 (considerando $\{1\}$ no lugar de $T, T$ no lugar de $S$ e $M=\mathbb{Z}_{2}$ ), obtemos o $\mathbb{Z}_{2} T$-isomorfismo

$$
\mathbb{Z}_{2} G \simeq \ln d_{\{1\}}^{G} \mathbb{Z}_{2} \simeq \bigoplus_{g \in T \backslash G} \operatorname{Ind} d_{\{1\}}^{T} g \mathbb{Z}_{2}
$$

onde $T \backslash G$ é um conjunto de representantes para as classes laterais à direita de $T$ em $G$. Daí,

$$
\begin{aligned}
& H^{1}\left(T ; \mathbb{Z}_{2} G\right) \simeq H^{1}\left(T ; \bigoplus_{g \in T \backslash G} \operatorname{Ind}_{\{1\}}^{T} g \mathbb{Z}_{2}\right) \\
& \simeq \bigoplus_{g \in T \backslash G} H^{1}\left(T ; \operatorname{In} d_{\{1\}}^{T} g \mathbb{Z}_{2}\right) \quad \text { (por III.5.3, pois } T \text { éf.g.) } \\
& \simeq \bigoplus_{g \in T \backslash G} H^{1}\left(T ; \mathbb{Z}_{2} T\right) \\
& =0 \quad \text { (por 11.4.10, pois } e(T)=1 \text { ). }
\end{aligned}
$$

Logo, her $r \epsilon s_{S, \mathbf{Z}_{2} G}^{G}=H^{1}\left(G ; \mathbb{Z}_{2} G\right)$, e portanto

$$
E\left(G, T, \mathbb{Z}_{2} G\right)=1+\operatorname{dim} H^{1}\left(G ; \mathbb{Z}_{2} G\right)=e(G) .
$$

Daí o resultado segue. 
Uma vez que $\mathcal{F}_{S} G \simeq \operatorname{In} d_{S}^{G} \overline{\mathbf{Z}_{2} S}$ e $\mathcal{F}_{S} T \simeq \operatorname{In} d_{S}^{T} \overline{\mathbf{Z}_{2} S}$, considerando $M=\overline{\mathbf{Z}_{2} S}$ no corolário 11.2.9, temos:

2.7 Lema: Sejam $S$ e $T$ subgrupos de $G$ satisfazendo $S \leq T \leq G$. Se $[G: S]=\infty$ e $[G: T]<\infty$ entâo

$$
\dot{E}(G, S) \leq \hat{E}(T, S)
$$

Como último resultado desta seç̧ào nós observamos que as propriedades de dualidade obtidas para $E(G, S)$ em 11.3 são preservadas para $\tilde{E}(G, S)$ embora tenhamos $\tilde{E}(G, S) \geq E(G, S)$.

2.8 Proposição: $S \epsilon j a ~(G: S)$ um par de grupos, com $[G: S]=\infty$.

(i) $S \epsilon(G, S)$ é um $D^{n}$-par entâo $\hat{E}(G, S)=1$.

(ii) $S \epsilon G$ é um $D^{n}$-grupo com módulo dualizante $C \in S$ é um $D^{n-1}$-subgrupo com módulo dualizante $\operatorname{Res}_{S}^{G} C$, entào $\hat{E}(G, S) \leq 2$.

(iii) Se $G$ é um $D^{n}$-grupo $(n>1)$ e hd $S \leq n-2$, entâo $\tilde{E}(G, S)=1$.

Demonstração: É análoga às demonstrações das proposições II.3.6 e 1l.3.9; basta lembrar que $\mathcal{F}_{S} G=\operatorname{Ind} G \overline{\mathbb{Z}_{2} S}$ e então trabalhar com $\overline{\mathbb{Z}_{2} S}$ no lugar de $\mathbb{Z}_{2}$.

Analizando os exemplos vistos no capítulo Il, secção 3, temos

2.9 Exemplos:

(i) Se $G=\langle a\rangle *\langle b\rangle \simeq \mathbb{Z} * \mathbb{Z}, S=\left\langle a b a^{-1} b^{-1}\right\rangle$ e $T=\langle b\rangle$, entâo $\hat{E}(G, S)=1$ e $\hat{E}(G, T)=\infty$.

(ii) Se $G=\mathbb{Z}^{k}$ e $S=\mathbb{Z}^{k-1}, k \geq 2$, então $E(G, S)=2$.

(iii) $\hat{E}\left(\mathbb{Z}^{k}, \mathbb{Z}^{r}\right)=1$ se $0 \leq r \leq k-2$.

(iv) $\hat{E}\left(<a, b ; a^{2} b^{2}=1>, S=\langle a\rangle\right) \leq 2$.

(v) Se $G, S$ e $K$ são como no exemplo II.3.10(c), entâo $\hat{E}(G, S)=2=$ $\tilde{E}(G, K)$.

(vi) Se $G$ e $S$ são como no exemplo Il.3.10(d), entào $\hat{E}(G, S)=1$. 
Para obter (ii) e (v) recordamos que nestes casos $S$ é normal em $G$, logo $E(G, S)=e(G, S)=e(G / S)=2$. Dai, por 2.1 e proposição anterior, temos $2=E(G, S) \leq \tilde{E}(G, S) \leq 2$.

Vejamos outros exemplos:

2.10 Exemplos: (i) Se $X=M^{2}-D^{2}$ onde $M^{2}$ é qualquer superfície fechada orientada diferente de $S^{2}$, entâo $\left(G=\Pi_{1}\left(X^{\prime}\right), S=\Pi_{1}(\partial X)\right)$ é um $P D^{2}$-par (por II.3.5) e portanto $\hat{E}(G, S)=1$.

(ii) Se $G=\mathbb{Z} \oplus(\mathbb{Z} * \mathbb{Z}) \oplus \mathbb{Z}_{n}$, onde $\mathbb{Z}_{n}$ é o grupo cíclico de ordem $n \geq 2$ e $S=\mathbb{Z} * \mathbb{Z}$ entào $\hat{E}(G, S)=2$.

De fato, visto que $\mathbb{Z}$ é um $P D^{1}$-grupo e $S=\mathbb{Z} * \mathbb{Z}$ é um $D^{1}$-grupo (com módulo dualizante $C \simeq H^{1}\left(S ; \mathbb{Z}_{2} S\right)$ ) temos, por 1.5.19, que $T=\mathbb{Z} \oplus(\mathbb{Z} * \mathbb{Z})$ é um $D^{2}$-grupo com módulo dualizante $\mathbb{Z}_{2} \otimes C: \simeq C$. Dai, segue, de 2.7 e proposição anterior (ii), que

$$
\tilde{E}(G, S) \leq \tilde{E}(T, S) \leq 2 .
$$

Agora, como $S$ é um subgrupo normal em $G$ temos, por II.4.24, que

$$
E(G, S)=\epsilon(G, S)=\epsilon(G / S)=\epsilon\left(\mathbb{Z} 巴 \mathbb{Z}_{r_{n}}\right)=\epsilon(\mathbb{Z})=2 .
$$

Logo, por $2.21,2=E(G, S) \leq \hat{E}(G, S) \leq 2$ e portanto, $\hat{E}(G, S)=2$.

\section{3. $\dot{\mathrm{E}}(\mathrm{G}, \mathrm{S})$ e o Invariante end $\dot{\mathrm{e}}(\mathrm{G}, \mathrm{S})$.}

Nesta seç̧âo analizaremos a relaçâo entre os invariantes $\tilde{E}(G, S)$ e $\tilde{e}(G, S)$. O invariante $\tilde{\epsilon}(G, S)$ foi definido por Liropholler e Roller implicitamente em [21] (1988) e explicitamente em [22] (1989).

Com o objetiro de facilitar ao leitor recordamos aqui a definiçâo deste invariante e apresentamos (sem demonstração) algumas de suas propriedades. Para maiores detalhes e resultados adicionais ver [22] 
3.1 Definição: Seja $(G, S)$ um par de grupos (com $[G, S]$ não necessariamente infinito). Então por definição,

$$
\tilde{\boldsymbol{e}}(G, S)=\operatorname{dim} H^{0}\left(G ; P G / \mathcal{F}_{S} G\right)=\operatorname{dim}\left(P G / \mathcal{F}_{S} G\right)^{G} .
$$

3.2 Lema: [22, lema 1.2] $S \epsilon[G: S]=\infty$ entâo

$$
\grave{\epsilon}(G, S)=1+\operatorname{dim} H^{1}\left(G ; \mathcal{F}_{S} G\right) .
$$

3.3 Lema: [22, lema 2.4]

(i) $\hat{e}(G,\{1\})=\epsilon(G)$ e mais geralmente $\tilde{e}(G, F)=e(G)$ se $F$ é um subgrupo

finito $d \varepsilon G$.

(ii) $\bar{\epsilon}(G, S)=0$ st, $\epsilon$ somente st, $[G: S]<\infty$.

(iii) Se $S \leq T \leq G \in[G: T]<\infty$, entâo $\bar{\epsilon}(G, S)=\tilde{e}(T, S)$.

(iv) Se $S \dot{e}$ finitamente gerado $\epsilon$ normal $\epsilon m G$, entâo $\bar{e}(G, S)=e(G / S)$.

(v) $S \epsilon S \leq T \leq G \in[G: T]=\infty$, então $\tilde{\epsilon}(G, S) \leq \tilde{e}(G, T)$, em particular, considerando $S=\{1\}$ obtemos $\epsilon(G) \leq \hat{\epsilon}(G, T)$.

3.4 Lema: [22, lema 2.5] $e(G, S) \leq \hat{e}(G, S)$.

3.5 Proposição: [22, corol. 4.3] Seja $G$ um $P D^{n}$-grupo e $S$ um $D^{n-1}$ subgrupo. Então $\hat{\epsilon}(G, S)=\infty$, ou $S \dot{\epsilon}$ um $P D^{n-1}$-grupo $\epsilon$, neste caso, $\hat{\epsilon}(G, S)=2$ (ou seja, $\left.H^{1}\left(G, \mathcal{F}_{S} G\right) \simeq \mathbb{Z}_{2}\right)$.

Nós podemos observar que algumas propriedades anteriormente obtidas para $\hat{E}(G, S)$ sào semelhantes às (respectivas) de $\hat{\epsilon}(G, S)$. Dai uma pergunta natural que surge é: que relaçâo existe entre tstes invariantes?. É claro que eles em geral nào sâo iguais pois, se isto ocorresse, teríamos por 3.4 , que $\epsilon(G, S) \leq \hat{E}(G, S)$ mas, como observamos em 2.4, não existe nenhuma relação entre estes invariantes. Além disto temos:

3.6 Observação: A proposição $2.8(\mathrm{i})$, nào é válida para $\tilde{\epsilon}(G, S)$ pois, para o $P D^{2}$-par $\left(G=\langle a\rangle *\langle b\rangle, S=\left\langle a b a^{-1} b^{-1}\right\rangle\right)$ temos que $\tilde{e}(G, S)=\infty$ uma vez que por $3.3(v), \infty=\epsilon(G) \leq \tilde{\epsilon}(G, S)$. 
Na realidade, segue de $3.3(\mathrm{v})$, que $\tilde{e}(G, S)=\infty$ scmpre que $e(G)=\infty$ (independentemente de $S$ ). Portanto $\tilde{e}(G, S)$ nào nos dá nenhuma informaçâo a respeito de $S$ quando $\epsilon(G)=\infty$, o que nâo ocorre para o invariante $\hat{E}(G, S)$. Por exemplo para $G=\langle a\rangle *\langle b\rangle, S=\left\langle a b a^{-1} b^{-1}\right\rangle$ e $T=\langle b\rangle$ temos, por 2.9(j) que $\hat{E}(G, S)=1 \neq \hat{E}(G, T)=\infty$.

3.7 Observação: A definiçào 3.1 é equivalente à $\bar{\epsilon}(G, S)=\operatorname{dim} \frac{\mathcal{A}_{S} G}{\mathcal{F}_{S} G}$, onde $\mathcal{A}_{S} G$ e como em 1.4. Por outro lado, vimos na secçào l, que

$$
\tilde{E}(G, S)=\operatorname{dim} \frac{\mathcal{A}_{S} G \cap(P G)^{S}}{\mathcal{F}_{S} G \cap(P G)^{S}}
$$

Logo, temos:

$$
\tilde{e}(G, S)=\tilde{E}(G, S) \quad \text { se, e somente se, } \quad \operatorname{dim} \frac{\mathcal{A}_{S} G}{\mathcal{F}_{S} G}=\operatorname{dim} \frac{\mathcal{A}_{S} G \cap(P G)^{S}}{\mathcal{F}_{S} G \cap(P G)^{S}} .
$$

Na proposiçâo seguinte comparamos os dois invariantes sob outro aspecto.

3.8 Proposição: Seja $(G, S)$ um par de grupos. Entâo,

(i) $\tilde{E}(G, S) \leq \tilde{e}(G, S)$.

(ii) Suponhamos que $[G: S]=\infty$. Se res $s_{S}^{G}: H^{1}\left(G ; \mathcal{F}_{S} G\right) \rightarrow H^{1}\left(S ; \mathcal{F}_{S} G\right)$ é a aplicação nula entâo $\hat{E}(G, S)=\bar{\epsilon}(G, S)$. (Em particular, $s \in H^{1}\left(S ; \mathcal{F}_{S} G\right)=0$ entâo isto ocorre). Se $\dot{E}(G, S)=\hat{\epsilon}(G, S)<\infty$, vale a recíproca deste fato.

\section{Demonstração:}

(i) Se $[G: S]<\infty$ entào, por 1.7 e $3.3(\mathrm{i}), \hat{E}(G, S)=0=\hat{e}(G, S)$. Suponhamos $[G: S]=\infty$. Claramente

$$
\hat{E}(G, S)=1+\operatorname{dim} \text { her } r \epsilon s_{S}^{G} \leq 1+\operatorname{dim} H^{1}\left(G ; \mathcal{F}_{S} G\right)
$$

pois Ker $r \epsilon s_{S}^{G} \subset H^{1}\left(G ; \mathcal{F}_{S} G\right)$. Dai o resultado segue de 3.2.

(ii) $r \epsilon s_{S}^{G}$ é nula $\Leftrightarrow$ lier $r \epsilon s_{S}^{G}=H^{1}\left(G ; \mathcal{F}_{S} G\right) \stackrel{(1)}{\Rightarrow} \operatorname{dim}$ her $r \epsilon s_{S}^{G}=$ $=\operatorname{dim} H^{1}\left(G ; \mathcal{F}_{S} G\right) \Leftrightarrow \hat{E}(G, S)=\hat{\epsilon}(G, S)$. Agora, se $\hat{E}(G, S)=\hat{\epsilon}(G, S)<\infty$ entâo (1) é válida na direção contrária.

Como consequencia deste resultado obtemos: 
3.8 Corolário: Scja $(G, S)$ um par de grupos com $[G: S]=\infty$. Se $S \dot{\epsilon}$ finitamente gerado e normal em $G$ entào

$$
\dot{E}(G, S)=\dot{\epsilon}(G, S)=c(G / S) .
$$

Demonstração: Se $S$ é normal em $G$, temos por 1.3.9 (Fórmula de Mackey - caso particular), o seguinte $\mathbf{Z}_{2} S$-isomorfismo

$$
\operatorname{Ind} d_{S}^{G} P S \simeq \bigoplus_{g \in G / S} g P S
$$

Daí, como $S$ é finitamente gerado temos (por 1.5.3) que

$$
H^{1}\left(S ; \mathcal{F}_{S} G\right)=H^{1}\left(S ; \bigoplus_{g \in G / S} g P S\right) \simeq \bigoplus_{g \in G / S} H^{1}(S ; g P S)
$$

Agora, recordemos (ver comentário anterior à 1.3.9), que $g P S$ é um $\mathbb{Z}_{2} S$ módulo com a $S$-ação dada por $s *(g A)=g s g^{-1} \cdot g A=g s A$, para todo $s \in S, A \in P S$, e $P S$ é um $\mathbb{Z}_{2} S$-módulo com a $S$-aşào dada pela multiplicação à esquerda. Considere

$$
\gamma: g P S \rightarrow P S \quad(g A \rightarrow A) .
$$

Claramente $\gamma$ é uma bijeção. Além disto, $\gamma$ é um $\mathbb{Z}_{2} S$-homomorfismo pois $\gamma(s * g A)=$ $\gamma(g s A)=s A=s \gamma(g A)$. Logo, para todo $g \in G / S$, temos:

$$
H^{1}(S ; g P S) \simeq H^{1}(S ; P S)=0 \quad(\text { por } 1.3 .16) .
$$

consequentemente, $H^{1}\left(S ; \mathcal{F}_{S} C_{i}\right)=0$ e entào, pela proposiçào anterior e $3.3(\mathrm{iv})$ tem-se o resultado desejado.

Nota: Não sabemos se a igualdade $\hat{E}(G, S)=\tilde{e}(G, S)$ é verdadeira quando $S$ é normal em $G$, não necessariamente finitamente gerado.

3.10 Corolário: Se $S$ é um subgrupo finito de $G \in[G: S]=\infty$, entâo

$$
\hat{E}(G, S)=\tilde{\epsilon}(G, S)=\epsilon(G)
$$


Demonstração: Se $S$ é finito temos que $\mathcal{F}_{S} G=F G \simeq \mathbf{Z}_{2} G \simeq \operatorname{Ind} d_{\{1\}}^{G} \mathbb{Z}_{2}$. Aplicando 1.3 .9 para $T=\{1\}$, obtemos o $\mathbf{Z}_{2} S$-isomorfismo

$$
\operatorname{In} d_{\{1\}}^{G} \mathbb{Z}_{2} \simeq \bigoplus_{g \in S \backslash G} \operatorname{In} d_{\{1\}}^{S} g \mathbf{Z}_{2}
$$

Consequentemente, teremos

$$
\begin{array}{rlr}
H^{1}\left(S ; \mathcal{F}_{S} G\right) & \simeq H^{1}\left(S ; \oplus \operatorname{Ind} d_{\{1\}}^{S} g \mathbb{Z}_{2}\right) \\
& \simeq H^{1}\left(S ; \bigoplus_{g \in S \backslash G} \operatorname{Coind}_{\{1\}}^{S} g \mathbb{Z}_{2}\right) & \text { (por Il.3.6, pois }[S:\{1\}]<\infty) \\
& \simeq \bigoplus_{g \in S \backslash G} H^{1}\left(S ; \operatorname{Coind} d_{\{1\}}^{S} g \mathbb{Z}_{2}\right) & \text { (por IlI.5.3, pois } S \text { é f.g.) } \\
& \simeq \bigoplus_{g \in S \backslash G} H^{1}\left(\{1\} ; g \mathbb{Z}_{2}\right) & \text { (por lema de Shapiro - Il.3.14) } \\
& =0 .
\end{array}
$$

Logo, pela proposiçâo anterior e $3.3(\mathrm{i})$, obtemos as igualdades $\dot{E}(G, S)=\hat{\epsilon}(G, S)=\epsilon(G)$

Nota: Se $\epsilon(G)=2$, pode-se verificar que para todo subgrupo $S$ de $G$ tem-se $[G: S]<\infty$ (e portanto $\tilde{E}(G, S)=\hat{\epsilon}(G, S)=0$ ) ou $|S|<\infty$ (e daí, pelo resultado anterior, $\hat{E}(G, S)=\hat{\epsilon}(G, S)=\epsilon(G)=2)$. Asssim no caso em que $\epsilon(G)=2$, a igualdade $\hat{E}(G, S)=\hat{\epsilon}(G, S)$ é válida para qualquer subgrupo $S$.

3.11 Corolário: $S \epsilon j a(G, S)$ um parde grupos. $S \epsilon S$ é um $P D^{n}$-grupo, $\epsilon\left\{g \in G \mid c d\left(S^{g} \cap S\right)=n-1\right\}=\emptyset$, entâo $\tilde{E}(G, S)=\tilde{\epsilon}(G, S)$.

Demonstração: Por [21, lema 5.3], $H^{1}\left(S: \mathcal{F}_{S} G\right)=0$. Daí, basta aplicar a proposiçào anterior.

Como uma aplicaşão deste resultado temos:

3.12 Exemplo: Seja $G$ o $P D^{3}$-grupo $\mathbb{Z} \subseteq(\mathbb{Z} \rtimes \mathbb{Z})=\Pi_{1}\left(S^{1} \times K B\right)$ onde $K B$ é a superficie da garrafa de Klein. Considere $S=\{(a, 0, c) \mid a, c \in \mathbb{Z}\}$. Entâo $S$ é um subgrupo de $G$ (nào normal) satisfazendo $\hat{E}(G, S)=\hat{\epsilon}(G, S)=2$. 
De fato, a operaçào em $G$ é dada por $(a, b, c)+(x, y, z)=$ $\left(a+x, b+(-1)^{c} y, c+z\right)$. Dai, claramente $S$ é um subgrupo de $G$ e portanto $S \simeq \mathbf{Z} \oplus \mathbf{Z}$ é um $P D^{2}$-grupo. Vejamos que $\left\{g \in G \mid c d\left(S^{s} \cap S\right)=1\right\}=\emptyset$.

Se $g \in S$ entào $c d\left(S^{g} \cap S\right)=2$ pois $S^{g} \cap S=S$.

Seja $g=(a, b, c)$ um elemento qualquer de $G-S$ (portanto $b \neq 0$ ). Podemos ver $S=S_{0} \cup S_{1}$ onde $S_{0}=\{(x, 0,2 k) \mid x, k \in Z\}$ e $S_{1}=\{(x, 0,2 k+1) \mid x, k \in \mathbb{Z}\}$. Daí $S^{g} \cap S=\left(S_{0} \cup S_{1}\right)^{g} \cap S=S_{0}^{g} \cap S \cup S_{1}^{g} \cap S$. Se $u=(x, 0,2 k) \in S_{0}$, entào

$$
\begin{aligned}
g+u+(-g) & =(a, b, c)+(x, 0,2 k)+\left(-a,-(-1)^{c} b,-c\right) \\
& =(a+x, b, c+2 k)+\left(-a,(-1)^{c+1} b,-c\right) \\
& =\left(a+x+(-a), b+(-1)^{c+2 k}(-1)^{c+1} b, c+2 k+(-c)\right) \\
& =(x, 0,2 k)=u \in S_{0} .
\end{aligned}
$$

Assim, $S_{0}^{g} \cap S=S_{0} \cap S=S_{0}$ se $g \in G-S$.

Por outro lado, se $u=(x, 0,2 k+1) \in S_{1}$, entào

$$
\begin{aligned}
g+u+(-g) & =(a, b, c)+(x, 0,2 k+1)+\left(-a,-(-1)^{c} b,-c\right) \\
& =(a+x, b, c+(2 k+1))+\left(-a,-(-1)^{c+1} b,-c\right) \\
& =\left(a+x-a, b+(-1)^{c+2 k+1}(-1)^{c+1} b, c+2 k+1-c\right) \\
& =(x, b+b, 2 k+1) \\
& =(x, 2 b, 2 k+1) \notin S \quad(\quad \text { pois } b \neq 0)
\end{aligned}
$$

Portanto $\quad S_{1}^{e} \cap S=\emptyset$. Logo se $g \in G-S$ entào $S^{g} \cap S=S_{0} \simeq \mathbb{Z} \oplus \mathbb{Z}$ e dá $c d\left(S^{9} \cap S\right)=c d\left(S_{0}\right)=2$.

Temos então que $G$ é um $P D^{3}$-grupo. $S$ é um $P D^{2}$-subgrupo e $\left\{g \in G \mid c d\left(S^{g} \cap S\right)=1\right\}=\emptyset$. Pelo corolário anterior e proposição 3.5 , obtemos $\hat{E}(G, S)=\hat{\epsilon}(G, S)=2$.

3.13 Observação: Uma vez que

$$
\hat{E}(G, S)=1+\operatorname{dim} \frac{H^{1}\left(G, S ; \mathcal{F}_{S} G\right)}{P\left(G, S \cdot \mathcal{F}_{S} G\right)}
$$

sempre que ocorrer a igualdade $\hat{E}(G, S)=\hat{c}(G, S)$, obteremos (compare com II.4.20) uma interpretação para $\hat{\varepsilon}(G, S)$ em termos de cohomologia relativa. 
Nas hipóteses do lema abaixo esta fórmula torna-se mais simples.

3.14 Proposição: $\operatorname{Seja}(G, S)$ um par grupo com $[G: S]=\infty$. Se $S$ é um $P D^{n}$-subgrupo com $n>1$, malnormal em $G$ (isto é, $S^{g} \cap S=\{1\}, \forall g \in G-S$ ) então

$$
\dot{\epsilon}(G, S)=\tilde{E}(G, S)=\operatorname{dim} H^{1}\left(G, S ; \mathcal{F}_{S} G\right) \text {. }
$$

Demonstração: Considere a sequência exata 2.2(1) para $M=\mathcal{F}_{S} G$, onde já estamos levando em conta o fato que $H^{0}\left(G ; \mathcal{F}_{S} G\right)=0$ pois $[G: S]=\infty$,

$$
0 \rightarrow H^{0}\left(S ; \mathcal{F}_{S} G\right) \rightarrow H^{1}\left(G, S ; \mathcal{F}_{S} G\right) \stackrel{\lrcorner}{\rightarrow} H^{1}\left(G ; \mathcal{F}_{S} G\right) \stackrel{\operatorname{res}_{S}^{G}}{\rightarrow} H^{1}\left(S ; \mathcal{F}_{S} G\right) \rightarrow \cdots
$$

Seja $E$ um conjunto de representantes para as classes laterais duplas $S g S$ onde $g \in G($ com $l \in E)$. Temos

$$
\begin{array}{rlr}
H^{0}\left(S ; \mathcal{F}_{S} G\right) & \simeq H^{0}\left(S ; \operatorname{In} d_{S}^{G} P S\right) \\
& \simeq H^{0}\left(S ; \bigoplus_{g \in E} I n d_{S \cap S_{s}}^{S} g P S\right) & \\
& \simeq \bigoplus_{g \in E} H^{0}\left(S ; \operatorname{In} d_{S \cap S_{s} g}^{S} g P S\right) & (\text { por } 11.3 .9, \text { com } T=S \text { e } M=P S)
\end{array}
$$

Como $S$ é malnormal em $G, S \cap S^{g}=\{1\}$ para todo $g \in G-S$. Daí, se $g \in E$ e $g \neq 1$, entào $H^{0}\left(S ; \operatorname{In} d_{S \cap S g}^{S} g P S\right)=H^{0}\left(S ; \operatorname{Ind} d_{\{1\}}^{S} g P S\right)=0$, pois $[S:\{1\}]=\infty$ (1.3.11). Agora, para $g=1 \in E, \quad H^{0}\left(S ; \ln _{S \cap S_{s}}^{S} g P S\right)=H^{0}(S ; P S)=\mathbb{Z}_{2}$. Dai, $H^{0}\left(S ; \mathcal{F}_{S} G\right)=\mathbb{Z}_{2}$ e portanto obtemos, da sequência (1) acima, a seguinte sequência exata curta:

$$
0 \rightarrow \mathbb{Z}_{2} \rightarrow H^{1}\left(G, S ; \mathcal{F}_{S} G\right) \rightarrow \operatorname{Im} J=\operatorname{ker} r \epsilon s_{S}^{G} \rightarrow 0 .
$$

Logo, $\operatorname{dim} H^{1}\left(G, S ; \mathcal{F}_{S} G\right)=1+\operatorname{dim} \operatorname{ker} r e s_{S}^{G}=\hat{E}(G, S) . \quad$ Para obter a igualdade $\tilde{E}(G, S)=\tilde{\epsilon}(G, S)$, observe que $c d\left(S^{g} \cap S\right)=\left\{\begin{array}{ll}n & \text { se } g \in S \\ 0 & \text { se } g \in G-S,\end{array}\right.$ e consequentemente, $\left\{g \in G \mid c d\left(S^{9} \cap S\right)=n-1\right\}=\emptyset$ (pois $n>1$ ). Daí o corolário 3.11 se aplica.

Podemos notar que em todos os exemplos vistos, $\hat{E}(G, S)=0,1,2$ ou $\infty$. Assim, uma questâo interessante é: Quais valores $\tilde{E}(G, S)$ pode assumir? Para $\tilde{e}(G, S)$

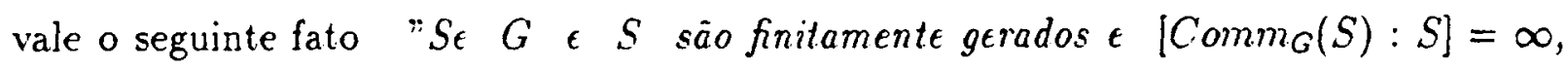


onde $\operatorname{Comm}_{G}(S)=\left\{g \in G \mid\left(S: S \cap S^{\theta}\right)<\infty \quad \& \quad\left(S^{g}: S \cap S^{g}\right)<\infty\right\}$, então $\tilde{e}(G, S)=1,2$ ou $\infty$ (cf. $[22$, teor. 1.3]). Nâo sabemos se vale um resultado similar para $\dot{E}(G, S)$. No entanto, de acordo com [22, prop. 4.7], existem pares $(G, S)$ para os quais $\hat{e}(G, S)$ toma valores distintos de $0,1,2$, ou $\infty$. Nós observamos que na demonstração deste resultado os autores provaram que $H^{1}\left(S ; \mathcal{F}_{S} G\right)=0$. Daí, por 3.8(ii), isto também é verdadeiro para $\hat{E}(G, S)$, ou seja:

3.15 Proposição: $S \epsilon G=G_{1}{ }_{S} G_{2}$ é um produto livre amalgamado (ver def. IV.1.1 (i)) onde

(a) $\left(G_{1},\{S\}\right)$ é um $P D^{3}$-par.

(b) $S$ é abeliano livre de posto dois.

(c) $S$ é malnormal em $G_{1}$.

(d) $G_{2} \dot{\epsilon}$ abeliano livre de posto dois $\epsilon S$ tem índice $n \in m G_{2}$.

entâo

$$
\hat{E}(G, S)=\hat{e}(G, S)=n
$$

Finalizando a seçâo, observamos que, tomando $M=\mathcal{F}_{S} G$ em II.5.2, obtemos uma interpretação topológica para $\hat{E}(G, S)$ em termos de pares Eilenberg-MacLane e consequentemente $\hat{\epsilon}(G, S)$ terá tal interpretaçâo quando a igualdade $\hat{\epsilon}(G, S)=\hat{E}(G, S)$ for verdadeira.

3.16 Proposição: Sejam $G$ em grupo; $S$ um subgrupo de $G$ com $[G: S]=\infty$. Se $(X, Y)$ é um par Eilenberg-MacLane que realiza topologicamente $(G, S) \in \hat{\epsilon}(G, S)=\hat{E}(G, S)$ entào

$$
\tilde{\epsilon}(G, S)=1+\operatorname{dim} \frac{H^{1}\left(X, Y ; \mathbb{Z}_{2}(G / S)\right)}{\left(\mathbb{Z}_{2}(G / S)\right)^{S}},
$$

onde $H^{1}\left(X, Y ; \mathbb{Z}_{2}(G / S)\right)$ é tomada com coeficientes locais. 


\section{CAPÍtULO IV}

\section{$\tilde{\mathbf{E}}(\mathbf{G}, \mathbf{S})$ e decomposição de grupos}

Dados $G$ um grupo e $S$ um subgrupo de $G$ com $G$ e $S$ finitamente gerados, Kropholler e Roller em [21] supondo que $H^{1}\left(G ; \mathcal{F}_{S} G\right) \simeq \mathbb{Z}_{2}$ ou equivalentemente, $\tilde{\epsilon}(G, S)=2$ (cf. Ill.3.2) apresentaram uma condiçâo necessária e (sob algumas hipóteses) suficiente, para que $G$ admita uma decomposição sobre um subgrupo comensurável com $S$. A condiçâo é que uma obstrução " $\operatorname{sing} g_{G}(S)$ ", definida pelos autores, seja nula.

Neste capítulo analisamos alguns dos resultados deste artigo em termos do nosso invariante $\dot{E}(G, S)$. Observamos, por exemplo, que nosso invariante $\tilde{E}(G, S)$ substitui a obstruçào $\operatorname{sing}(S)$. Assim, nas hipóteses consideradas, $\tilde{E}(G, S)$ é suficiente para decidir se $G$ se decompòe ou nào sobre um subgrupo comensurável com $S$ (2.13).

É nosso interesse sempre que possivel retirar dos resultados de [21] a hipótese geral de que $\tilde{e}(G, S)=2$. Neste sentido nós verificamos que o seguinte resultado é válido (sem tal hipótese).

"Se $G$ se decompòe sobre um subgrupo comensurável com $S$ entâo $\tilde{E}(G, S) \geq 2$ " (cf. Teorema 2.6).

Como consequência deste fato concluímos (ver 2.7) que se $(G, S)$ é um $D^{n}$. par então $G$ não se decompõe sobre um subgrupo comensurável com $S$. Isto nos dá exemplos de pares de grupos $(G, S)$ tais que $e(G, S)=\hat{\epsilon}(G, S)=\infty$ e no entanto $G$ não se decompõe sobre subgrupo comensurável com $S$ (ver observaçâo 2.8).

Em um outro resultado analisado, verificamos que a hipótese $H^{1}\left(G, \mathcal{F}_{S} G\right) \simeq$ $\mathbf{Z}_{2}$ é consequência das demais e portanto não é possível suprimi-la (2.11). 
Isto tudo é feito na seç̧ào 2. Inicialmente na secçào 1 nós recordamos algumas definiçōes e resultados (sem demonstraçōes) existentes para decomposiçào de grupos envolvendo os invariantes ends $e(G)$ e $e(G, S)$.

É interessante observar que a recíproca do teorema 2.6 é um problema ainda em aberto.

\section{Decomposiçāo de grupos}

1.1 Definição: [4, 2.4 e 2.5]

(i) Sejam $H$ e $K$ grupos com subgrupos $S \leq H, T \leq K$ e suponhamos que $S$ e $T$ sâo isomorfos via $\theta: S \stackrel{\sim}{\rightarrow}$. Entâo o produto livre amalgamado $d \epsilon H$ $\epsilon K$ com subgrupo amalgamado $S \simeq T$ é definido por

$$
G=H *_{s} K:=<H, K ; r e l H, r e l K, s=\theta(s), \forall s \in S>\text {. }
$$

(ii) Seja $H$ um grupo com subgrupos isomorfos $S$ e $T$ e seja $\sigma: S \rightarrow T$ um isomorfismo dado. $O$ grupo HNN sobre o grupo base $H$ com subgrupo associado $S \simeq T$ e letra estável $p$ é definido por

$$
G=H *_{s, o}=<H, p ; r e l H, p s p^{-1}=\sigma(s) . \forall s \in S>.
$$

Em (i), dados os homomorfismos $j_{1}: H \rightarrow G$ e $j_{2}: K \rightarrow G$ induzidos pela inclusào, podemos provar que $j_{i}, i=1,2$ sào injetoras e $j_{1}(H) \cap j_{2}(K)=j_{1}(S)=j_{2}(T)$. Dai, usando $j_{i}$ como identificação, $i=1,2$, podemos considerar $H$ e $K^{\prime}$ como subgrupos de $G, \operatorname{com} S=H \cap K^{\prime}=T$.

Analogamente, em (ii), podemos considerar $H$ como subgrupo de $G$.

1.2 Definição; Dizemos que um grupo $G$ se decompõe sobre um subgrupo $S$ se $G=H *_{s} K$ com $H \neq S \neq K$ ou $G=H *$ *o.

1.3 Exemplos: (i) $\mathbb{Z}=\{1\} *\{1\}$, assim $\mathbb{Z}$ se decompóe sobre o subgrupo trivial.

(ii) Seja $G$ o grupo fundamental da superficie fechada orientada $M$ de genus 
2, e seja $S$ o grupo fundamental da sub-superfície $X$ de $M$ como na figura abaixo. Então $G$ se decompóe sobre $S$, pois $G=\Pi_{1}\left(X_{1} \cup X\right) *_{S} \Pi_{1}\left(X \cup X_{2}^{\prime}\right)$.

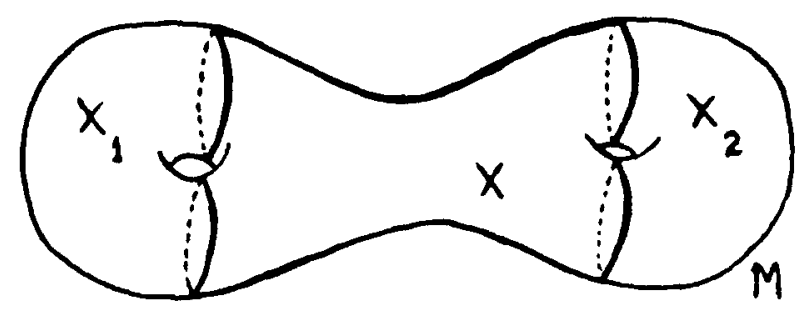

O primeiro resultado de decomposição de grupos envolvendo a teoria de ends foi dado por Stallings [31] (1968) e [32] (1971). Este é um resultado de decomposição sobre subgrupo finito e é conhecido como teorema de estrutura de Stallings.

1.4 Teorema: St $G$ é finitamente gerado, entāo $\epsilon(G) \geq 2$ se e somente se $G$ se decompôt sobre um subgrupo finito.

1.5 Observaçōes: (i) De fato, $e(G)=2 \Longleftrightarrow G=S *_{S}$, com $S$ finito ou $G=H *_{S} K$, com $S$ finito e $[H: S]=\left[K^{\prime}: S\right]=2$ ([32] ou [27, teor. 5.12]). E, $e(G)=\infty \Longleftrightarrow G=H *_{S} K$ com $S$ finito contido propriamente em ambos os fatores e de índice $>2$ em ao menos um deles, ou $G=H * s$, onde $S$ é finito, mergulhado propriamente em $H([32,5 . \mathrm{A} .10])$.

(ii) A implicaşâo: "G se decompōe sobre um subgrupo finito $\Longrightarrow$ $\epsilon(G) \geq 2$ ", é válida para $G$ não necessariamente finitamente gerado ([27, lema 6.3]).

No entanto, se por exemplo, estamos interessados em saber quando o grupo fundamental de uma superficie $F$ injeta no grupo fundamental de uma três variedade fechada e conexa $M$, caimos em um problema de $G=\Pi_{1}(M)$ se decompor sobre um subgrupo infinito $S=\Pi_{1}(F)$.

1.6: Scott en [26] (197i), tentou generalizar o resultado de Stallings acima para grupos que se decompôem sobre subgrupos infinitos. Ele mostrou que: "Se $G$ se decompôe sobre $S$ então $e(G, S) \geq 2 "$ (cf [26, lema 1.8]). O problema está na direçâo 
contrária. Scott esperava provar que: ${ }^{n} e(G, S) \geq 2$ se e somente se, $G$ se decompóe sobre alguma extensão finita $T$ de $S^{\prime \prime}$. No entanto ele observou que isto era falso em geral. $\mathrm{O}$ resultado obtido por Scott foj o seguinte:

1.7 Teorema: [26, teor. 4.1] Se $G$ e $S$ são grupos finitamente gerados $\epsilon G$ é S-residualmente finito (isto é, dado $g \in G-S$, existe um subgrupo $G_{1} d e$ indice finito em $G$ tal que $G_{1} \supset S$ mas $\left.g \notin G_{1}\right)$. Então $e(G, S) \geq 2$ se $\epsilon$ somente se, $G$ tem um. subgrupo $G_{2}$ de índice finito em $G$ tal que $G_{2} \supset S$ e $G_{2}$ se decompöe sobre $S$.

Dado $(G, S)$, estaremos interessados em investigar quando $G$ se decompóe sobre um subgrupo comensurável com $S$.

1.8 Definição: Dois subgrupos $S \in T d \epsilon G$ são ditos comensuráveis se $[S: S \cap T]<\infty \in[T: S \cap T]<\infty$.

Finalmente lembramos a correspondência entre decomposiçâo de grupos e ação de grupos sobre árvores (para majores detalhes sugerimos [28]). Os resultados de Kropholler e Roller sobre decomposição de grupos que iremos analisar na seç̧ão seguinte são baseados fortemente neste fato.

1.9 Proposição: Sejam $G$ um grupo $\epsilon S$ um subgrupo de $G$. Entâo $G$ se decompóe sobre $S$ se $\epsilon$ somente se existe uma G-árvone $Y$ tal que:

(i) $G$ atua livre de pontos fixos (isto $\dot{\epsilon}$, nenhum vértice de $Y \dot{\epsilon}$ fixado por todo o grupo $G$ ).

(ii) $G$ atua transitivamente $\epsilon$ sem inversão sobre as arestas $d \epsilon Y, \epsilon$

(iii) $S$ é o estabilizador de uma aresta.

\section{2. $\hat{E}(G, S)$ e decomposiçāo de grupos segundo Kropholler e Roller.}

Vejamos inicialmente a definiçâo da obstruçâo $\operatorname{sing}_{G}(S)$ introduzida por 
Kropholler e Roller em [21].

2.1 Definição: Seja $(G, S)$ um par grupo. Suponhamos que $H^{1}\left(G ; \mathcal{F}_{S} G\right) \simeq \mathbb{Z}_{2}$ com gerador $u$. Consideremos

$$
r e s_{S}^{G}: H^{1}\left(G ; \mathcal{F}_{S} G\right) \rightarrow H^{1}\left(S ; \mathcal{F}_{S} G\right),
$$

a aplicaşâo restriçâa. Então, por definiçâo,

$$
\sin g_{G}(S)=r \epsilon s_{S}^{G}(u) .
$$

Uma condição necessária para $G$ se decompor sobre um subgrupo comensurável com $S$ provada en [21] é a seguinte:

2.2 Proposição: [21, lema 2.4] Seja $(G, S)$ um par de grupos satisfazendo $H^{1}\left(G ; \mathcal{F}_{S} G\right) \simeq \mathbb{Z}_{2} \quad(\Leftrightarrow \dot{\epsilon}(G, S)=2)$, com $G$ e $S$ finitamente gerados. Se $G$ se decompôe sobre um subgrupo comensurável com $S$ entâo $\sin g_{G}(S)=0$.

Podemos observar que, nas hipóteses acima, as condiçôes $\sin g_{G}(S)=0 \mathrm{e}$ Ker $r \epsilon s_{S}^{G} \neq 0$ são equivalentes e entào temos:

2.3 Lema: $S \epsilon(G, S) \dot{\epsilon}$ um par grupo com $H^{3}\left(G ; \mathcal{F}_{S} G\right)=\mathbb{Z}_{2}$, então

(i) $\sin _{G}(S)=0 \Leftrightarrow \dot{E}(G ; S)=2$,

(ii) $\sin _{G}(S) \neq 0 \Leftrightarrow \dot{E}(G, S)=1$.

Demonstração: Se $H^{1}\left(G ; \mathcal{F}_{S} G\right) \simeq \mathbb{Z}_{2}$ então $[G, S]=\infty$. Dai $\tilde{E}(G, S)=$ $1+\operatorname{dim}$ Ker $r \epsilon s_{S}^{G}$ e portanto temos:

(i) $\sin _{G}(S)=0 \Leftrightarrow$ Ler $r \epsilon s_{S}^{G}=H^{1}\left(G, \mathcal{F}_{S} G\right)=\mathbb{Z}_{2} \Leftrightarrow \hat{E}(G, S)=2$.

(ii) $\sin _{G}(S) \neq 0 \Leftrightarrow$ lier $r \epsilon s_{S}^{G}=0 \Leftrightarrow \hat{E}(G, S)=1$.

Motivados neste lema simples, nós verificamos que, pensando em termos de $\tilde{E}(G, S)$ e não em termos de $\operatorname{sing}_{G}(S)$, o resultado 2.2 é válido sem a suposiçâo de que $H^{1}\left(G, \mathcal{F}_{S} G\right) \simeq \mathbb{Z}_{2}$ (ver proposição abaixo). 
Isto é obtido de maneira análoga à demonstraçào de 2.2 dada em [21], a qual decorre de alguns lemas.

Como as demonstraçōes destes lemas em [21] estâo bastante resumidas e portanto não muito claras, apresentamos aqui, em detalhes, as demonstraçöes dos lemas, adaptando-as ao invariante $\tilde{E}(G, S)$ e retirando a hipótese de que $H^{1}\left(G ; \mathcal{F}_{S} G\right)=\mathbf{Z}_{2}$.

2.4 Lema: Seja $S$ um subgrupo de $G$, com $S$ e $G$ finitamente gerados. As seguintes condiçôes sâo equivalentes:

(i) $\hat{E}(G, S) \geq 2$

(ii) Existe $[B]=B+\mathcal{F}_{S} G \in\left(\frac{P G}{\mathcal{F}_{S} G}\right)^{G}$ (isto é, $B+g B \in \mathcal{F}_{S} G, \forall g \in G$ ) tal que $[B] \neq[\emptyset],[B] \neq[G]$ є $S B=B$.

Demonstração: Seja $F \stackrel{\mathscr{Z}}{\rightarrow} \mathbb{Z}_{2}$ uma resolução projetiva de $\mathbb{Z}_{2}$ sobre $\mathbb{Z}_{2} G$. Então $F \rightarrow \mathbb{Z}_{2}$ é também uma resoluçào de $\mathbb{Z}_{2}$ sobre $\mathbb{Z}_{2} S$, que é projetiva pelo fato que $\mathbf{Z}_{2} G$ é $\mathbb{Z}_{2} S$-livre.

Da sequência exata

$$
0 \rightarrow \mathcal{F}_{S} G \stackrel{k}{\leftrightarrow} P G \rightarrow \frac{P G}{\mathcal{F}_{S} G} \rightarrow 0,
$$

obtemos o diagrama comutativo de complexos de cocadeias com linhas exatas:

$$
\begin{aligned}
& 0 \rightarrow \operatorname{Hom}_{G}\left(F, \mathcal{F}_{S} G\right) \rightarrow \operatorname{Hom}_{G}(F, P G) \rightarrow \operatorname{Hom}_{G}\left(F, \frac{P G}{\mathcal{F}_{S} G}\right) \rightarrow 0 \\
& \downarrow \downarrow \downarrow \\
& 0 \rightarrow \operatorname{Hom}_{\mathcal{S}}\left(F, \mathcal{F}_{S} G\right) \rightarrow \operatorname{Hom}_{\mathcal{S}}(F, P G) \rightarrow H \operatorname{Hom}_{\mathcal{S}}\left(F, \frac{P G}{\mathcal{F}_{S} G}\right) \rightarrow 0
\end{aligned}
$$

Dai por [8, 1.0.4], aplicando $H^{*}(-)$, e lembrando o conceito de cohomologia de grupos, obtemos o diagrama comutativo com linhas exatas

$$
\begin{aligned}
& 0 \rightarrow H^{0}\left(G ; \mathcal{F}_{S} G\right) \rightarrow H^{0}(G ; P G) \rightarrow H^{0}\left(G ; \frac{P G}{\mathcal{F}_{S} G}\right) \stackrel{\wp}{\rightarrow} H^{1}\left(G ; \mathcal{F}_{S} G\right) \rightarrow \cdots \\
& \downarrow \quad \downarrow i \quad \downarrow j \quad \downarrow r e s_{S}^{G} \\
& 0 \rightarrow H^{0}\left(S ; \mathcal{F}_{S} G\right) \rightarrow H^{0}(S ; P G) \rightarrow H^{0}\left(S ; \frac{P G}{\mathcal{F}_{S} G}\right) \stackrel{\circ}{\rightarrow} H^{1}\left(S ; \mathcal{F}_{S} G\right) \rightarrow \cdots
\end{aligned}
$$


Agora, tanto em (i) com em (ii), temos que $[G: S]=\infty$, e dai $H^{0}\left(G ; \mathcal{F}_{S} G\right)=$ $\left(I n d_{S}^{G} P S\right)=0$ (1.3.11). Também $H^{1}(G ; P G)=0$ pelo lema de Shapiro (1.3.14). Logo temos:

$$
\begin{aligned}
& 0 \rightarrow(P G)^{G} \stackrel{\beta}{\rightarrow}\left(\frac{P G}{\mathcal{F}_{S} G}\right)^{G} \stackrel{6}{\rightarrow} H^{1}\left(G ; \mathcal{F}_{S} G\right) \rightarrow 0 \\
& \downarrow i \quad \downarrow j \quad \downarrow r e s_{S}^{G} \\
& 0 \rightarrow\left(\mathcal{F}_{S} G\right)^{S} \hookrightarrow(P G)^{S} \stackrel{\circ}{\rightarrow}\left(\frac{P G}{\mathcal{F}_{S} G}\right)^{S} \stackrel{\circ}{\rightarrow} H^{1}\left(S ; \mathcal{F}_{S} G\right) \rightarrow \cdots
\end{aligned}
$$

Vejamos que (i) $\Longrightarrow$ (ii).

Se $\hat{E}(G, S)=1+\operatorname{dim} \operatorname{Ker} r \epsilon s_{S}^{G} \geq 2$ então existe $u \in H^{1}\left(G ; \mathcal{F}_{S} G\right), u \neq 0$ tal que $\operatorname{res}_{S}^{G} u=0$. Como $\delta$ é sobrejetora, existe $\left[B_{0}\right] \in\left(\frac{P G}{\mathcal{F}_{S} G}\right)^{G}$ tal que $u=\delta\left[B_{0}\right]$, com $\left[B_{0}\right] \notin \operatorname{Im} \beta=\{[\emptyset],[G]\}$ pois $\delta\left[B_{0}\right] \neq 0$ e $\operatorname{Im} \beta=\operatorname{Ker} \delta$.

Da comutatividade do diagrama temos $\left.\rho\left(j \mid B_{0}\right]\right)=\left(r \in s_{S}^{G} \circ \delta\right)\left[B_{0}\right]=$ $r \in s_{S}^{G} u=0$. $[B]=\alpha(B)=\left[B_{0}\right]$.

Daí $\left[B_{0}\right]=j\left[B_{0}\right] \in \operatorname{Ker} \rho=\operatorname{Im} \alpha$ e portanto existe $B \in(P G)^{S}$ tal que

É fácil ver que $(P G)^{S}=\{A \subset G \mid S A=A\}$.

Então temos $S B=B$ (pois $\left.B \in(P G)^{S}\right) ;[B] \in\left(\frac{P G}{\mathcal{F}_{S} G}\right)^{G}$ e $[B] \notin\{[\emptyset],[G]\}$ (pois $[B]=\left[B_{0}\right]$ e $\left[B_{0}\right]$ satisfaz estas condiçôes), isto é, obtemos (ii).

Para ver (ii) $\Longrightarrow(\mathrm{i})$, seja $[B] \in\left(\frac{P G}{\mathcal{F}_{S} G}\right)^{G}$ tal que $[B] \neq[\emptyset], \quad[B] \neq[G]$ e $S B=B$. Entâo $[B] \notin \operatorname{Im} \beta=\operatorname{Ker} \delta$ e portanto $u:=\delta([B]) \neq 0$, com $B \in(P G)^{S}$. Daí, pela comutatividade do diagrama temos

$$
r \in s_{S}^{G} u=r \epsilon s_{S}^{G}(\delta[B])=(\rho \circ j)([B])=\rho([B])=\rho(\alpha(B))=0 \text {. Logo. }
$$

Ker $r \epsilon s_{S}^{G} \neq 0$ de onde segue que $E(G, S) \geq 2$.

2.5 Lema: Se $T$ é comensurável com $S$ entào $\hat{E}(G, S) \geq 2$ se $\epsilon$ somente se $\hat{E}(G, T) \geq 2$.

Demonstração: É suficiente provar o seguinte fato:

(1) Se $H$ e $K$ sâo subgrupos de $G, \operatorname{com} K^{\prime} \leq H \leq G$ e $\left[H: K^{\prime}\right]=n<\infty$, entào $\hat{E}\left(G, H^{\prime}\right) \geq 2$ implica $\hat{E}(G, H) \geq 2$. 
Pois, uma vez provado (1), se $T$ é qualquer subgrupo de $G$ comensurável com $S$, isto é, $[S: T \cap S]<\infty$ e $[T: T \cap S]<\infty$ então, por corolário Ill.2.6, $\hat{E}(G, S) \leq \dot{E}(G, S \cap T)$ e $\hat{E}(G, T) \leq \dot{E}(G, S \cap T)$. Daí

$$
\hat{E}(G, S) \geq 2 \Longrightarrow \hat{E}(G, S \cap T) \geq 2 \stackrel{(1)}{\Longrightarrow} \hat{E}(G, T) \geq 2,
$$

e analogamente,

$$
\hat{E}(G, T) \geq 2 \Longrightarrow \hat{E}(G, S \cap T) \geq 2 \stackrel{(1)}{\Longrightarrow} \hat{E}(G, S) \geq 2 .
$$

Provemos então (1).

Se $\hat{E}\left(G, K^{\prime}\right) \geq 2$ entào pelo lema anterior, existe $B \subset G$ tal que $B+g B \in \mathcal{F}_{K} G, \quad \forall g \in G,[B] \neq[\emptyset],[B] \neq[G]$ e $K B=B$. Como $[H: K]<\infty$ temos que $\mathcal{F}_{K} G=\mathcal{F}_{H} G$. Logo

$$
B+g B \in \mathcal{F}_{H} G, \quad \forall g \in G .
$$

Seja $H_{0}=\left\{h_{1}, \ldots, h_{n}\right\}$ um conjunto de representantes para as classes laterais à esquerda de $K$ em $H$.

Temos

$$
\begin{aligned}
B+H_{0} B & =B+\left(h_{1} B \cup \ldots \cup h_{n} B\right) \subset\left(B+h_{1} B\right) \cup \ldots \cup\left(B+h_{n} B\right) \\
& \subset F_{1} H \cup \ldots \cup F_{n} H, \quad \operatorname{com} F_{i} \in F G, \quad i=1, \ldots, n \text { por }(*) \\
& =\left(F_{1} \cup \ldots \cup F_{n}\right) H
\end{aligned}
$$

Portanto $B+H_{0} B \in \mathcal{F}_{H} G$ e dai $[B]=\left[H_{0} B\right]$.

Seja $B_{0}:=H_{0} B$. Entâo:

(a) $B_{0}+g B_{0} \in \mathcal{F}_{H} G, \forall g \in G$, pois

$$
\begin{aligned}
B_{0}+g B_{0} & =H_{0} B+g H_{0} B=\left(H_{0} B+B\right)+\left(B+g H_{0} B\right) \\
& =\left(H_{0} B+B\right)+B+g\left(h_{1} B \cup \ldots \cup h_{n} B\right) \\
& =\left(H_{0} B+B\right)+B+\left(g h_{1} B \cup \ldots \cup g h_{n} B\right) \\
& \subset\left(B+H_{0} B\right)+\left(B+g h_{1} B\right)+\ldots+\left(B+g h_{n} B\right) \in \mathcal{F}_{H} G \quad \text { por }(*) .
\end{aligned}
$$

(b) $\left[B_{0}\right] \neq[\emptyset]$ e $\left[B_{0}\right] \neq[G]$ pois $\left[B_{0}\right]=[B]$ e $[B] \neq[\emptyset],[G]$.

(c) $H B_{0}=B_{0}$ pois, claramente $B_{0} \subset H B_{0}$ e, como $H H_{0} \subset H$ (pois $\left.H_{0} \subset H\right), H=h_{1} l \dot{U} \ldots \dot{U} h_{n} K=H_{0} K$ e $K B=B$, temos: 


$$
H B_{0}=H\left(H_{0} B\right) \subset H B=H_{0} K B=H_{0} B=B_{0} .
$$

Logo $B_{0}$ satisfaz as condiçôes do lema 2.4 (ii) para $G$ e $H$. Portanto $\hat{E}(G, H) \geq 2$.

Temos então o seguinte resultado:

2.6 Teorema: $S \in j a(G, S)$ um par grupo com $G \in S$ finitamente gerados. Se $G$ se decompóe sobre um subgrupo comensurável com $S$ entāo $\tilde{E}(G, S) \geq 2$.

Demonstração: Suponhamos que $G$ se decompóe sobre um subgrupo $T$ comensurável com $S$. Por 1.9, existe uma $G$-árvore $Y$ tal que:

(i) $G$ atua livre de pontos fixos sobre os vértices de $Y$,

(ii) $G$ atua transitivamente e sem inversão sobre as arestas de $Y$, e

(iii) $T$ é o estabilizador de uma aresta.

Seja $\epsilon$ uma aresta com estabilizador $T$, isto é, $T=\{g \in G \mid e g=\epsilon\}$. Se $e$ é removida de $Y$ entào obteremos dois pedaços disjuntos que denotaremos por $Y_{0}$ e $Y_{1}$.

Seja $B=\left\{g \in G \mid \epsilon g \in Y_{0}^{\prime}\right\}$. Claramente $T B=B$ pois, se $t \in T$ e $g \in B$ entào $e(t g)=(\epsilon t) g=\epsilon g \in Y_{0}$. Além disso, $B+g B \in \mathcal{F}_{T} G, \forall g \in G$ pois:

$$
\begin{aligned}
B-g B & =\left\{y \in G \mid \epsilon y \in \zeta_{0} \text { e } y \notin g B\right\} \\
& =\left\{y \in G \mid \epsilon y \in Y_{0} \text { e } g^{-1} y \notin B\right\} \\
& =\left\{y \in G \mid \epsilon y \in Y_{0} \text { e } \epsilon g^{-1} y \in Y_{\mathrm{J}} \cup\{\epsilon\}\right\} \\
& =\left\{y \in G \mid \epsilon y^{-1} \text { pertence ao menor caminho ligando } \epsilon \text { a } \epsilon g^{-1}\right\}
\end{aligned}
$$

(a última igualdade é obtida fazendo $y^{-1}$ atuar à direita no caminho que liga ey a $\mathrm{eg}^{-1} y$ ).

Como a açào é transitiva, existem $g_{1}, \ldots, g_{k} \in G$ tais que as arestas deste caminho sâo $\epsilon g_{1}, \ldots, \epsilon g_{k}$. Logo $\forall y \in B-g B, e y^{-1}=\epsilon g_{\imath}$ para algum $i \in\{1, \ldots, k\}$ e assim, $e=e g_{i} y$, isto é, $g_{i} y \in T$. Daí, existe $t \in T$ tal que $g_{i} y=t$ ou seja, $y=g_{i}^{-1} t$. Logo $B-g B \subseteq g_{1}^{-1} T \cup \ldots \cup g_{k}^{-1} T$ e portanto $B-g B \in \mathcal{F}_{T} G$.

Analogamente temos 


$$
\begin{aligned}
g B-B & =\{y \in G \mid y \in g B \text { e } y \notin B\} \\
& =\left\{y \in G \mid g^{-1} y \in B \text { e } y \notin B\right\} \\
& =\left\{y \in G \mid e g^{-1} y \in Y_{0} \text { e } e y \in Y_{1} \cup\{\epsilon\}\right\} \\
& =\left\{y \in G \mid \epsilon y^{-1} \text { pertence ao menor caminho ligando } e g^{-1} \text { a } e\right\}
\end{aligned}
$$

e pelo mesmo raciocínio acima obtemos que $g B-B \in \mathcal{F}_{T} G$.

Dai $B+g B=B-g B \cup g B-B \in \mathcal{F}_{T} G$.

Agora, que $[B] \neq[\theta]$ e $[B] \neq[G]$ segue do fato que a ação de $G$ sobre os vértices de $Y$ é livre de pontos fixos.

Portanto $B$ satisfaz a condição (ii) do lema 2.4 e daí $\tilde{E}(G, T) \geq 2$. Como $T$ é comensurável com $S$, segue do lema anterior, que $\hat{E}(G, S) \geq 2$.

Como consequência deste resultado temos:

2.7 Corolário: $S \epsilon$ (i) $(G, S)$ é um $D^{n}$-par com $[G: S]=\infty$ ou,

(ii) $G$ é um $D^{n}$-grupo e $h d S \leq n-2$

entâo $G$ nâo se decompóe sobre nenhum subgrupo comensurável com $S$.

Demonstração: Segue de III.2.8 e do teorema anterior.

2.8 Observação: $O$ teorema 2.6 é bastante interessante pois, por exemplo, para $G=\langle a\rangle *\langle b\rangle \simeq \mathbb{Z} * \mathbb{Z}$ e $S=\left\langle a b a^{-1} b^{-1}\right\rangle$ temos que $\tilde{e}(G, S)=\infty \mathrm{e}$ portanto a obstruçâo $\operatorname{sing}_{G}(S)$ nào está definida, mas apesar disto, visto que $(G, S)$ é um $P D^{2}$-par, concluímos do corolário anterior, que $G$ não se decompóe sobre nenhum subgrupo comensurável com $S$. Em particular, sobre nenhuma extensão finita de $S$.

Isto também vem confirmar que a idéia inicial de Scott "Se $\epsilon(G, S) \geq 2$ então $G$ se decompôe sobre uma extensão finita de $S$ " (ver 1.6) não é realmente válida pois, neste exemplo, $\epsilon(G, S)=\infty>2$.

Para analisar quando a condição $\sin _{G}(S)=0$ é suficjente para $G$ se decompor sobre um subgrupo comensurável com $S$, em [21] §3, os autores, admitindo as hipóteses gerais 
(a) $G$ é um grupo finitamente gerado de dimensão cohomológica no máximo $n$,

(b) $S$ é um $P D^{n-1}$-subgrupo de $G$,

(c) $H^{\mathbf{1}}\left(G ; \mathcal{F}_{S} G\right) \simeq \mathbf{Z}_{2}$,

(d) Todo subgrupo de indice infinito em $G$ tem dimensão cohomológica no máximo $n-1$, provaram o seguinte resultado:

2.9 Proposição: [21, lema 3.2] Se $\left[N_{G}(S): S\right]=\infty$, onde $N_{G}(S)$ denota o normalizador de $S$ em $G$, entào $G$ se decompõe sobre um subgrupo comensurável com $S$ se, e somente se, $\operatorname{sing}_{G}(S)=0$.

Era nosso interesse retirar a hipótese (c) e generalizar este fato substituindo, como no teorema 2.6, a condiçâao $\operatorname{sing}_{G}(S)=0$ por $\hat{E}(G, S) \geq 2$. Porém, nós provamos (Teorema 2.11 abaixo), que se $\left[N_{G}(S): S\right]=\infty$ então a hipótese (c) é consequência das outras e portanto nâo faz sentido retirá-la. Além disto, nós verificamos, que a hipótese (b) pode ser enfraquecida, por considerar

(b)': $S$ é um $D^{n-1}$-sulggrupo de $G$.

Para provar este resultado, nos usamos lema 3.1 de [21]. Para deixar claro que na demonstraçào deste lema não se faz uso da hipótese (c) e que a hipótese (b) pode ser substituída por (b)', apresentaremos aquui demonstraçâo em detalhes deste lema.

2.10 Lema: Scja $(G, S)$ um par de grupos satisfazendo as condições (a), (b)' $e$ (d) acima. $S \epsilon\left[N_{G}(S): S\right]=\infty$ então

(i) $\left[G: N_{G}(S)\right]$ é finito, $\boldsymbol{\epsilon}$

(ii) $N_{G}(S) / S$ tem um subgrupo ciclico infinito $L / S$ de indice finito.

Demonstração: Por simplicidade vamos denotar $N_{G}(S)$ por $N$. Provemos inicialmente que:

(1) $N / S$ tem um subgrupo $H / S$ finitamente gerado, com cd $H=n n$.

De fato: Suponhamos, por absurdo, que para todo subgrupo finitamente 
gerado $H / S$ de $N / S$ tenhamos cd $H \neq n$.

Então $c d H=n-1$ pois, como: $c d S=n-1, c d G \leq n$ e $G \supset H \supset S$, as únicas possibilidades são, em vista de $1.5 .10, c d H=n$ ou $c d H=n-1$.

Agora, se $c d H=n-1$, visto que $S$ é normal em $H$ e $S$ é um $D^{n-1}$-grupo, segue de 1.5.24(a), que $H$ é também um $D^{n-1}$-grupo e $Q=H / S$ é finito.

Portanto se $H / S$ é um subgrupo finitamente gerado qualquer de $N / S$ então $H / S$ é finito ou seja, $N / S$ é localmente finito. Em particular, como por hipótese do lema $N / S$ não é finito, segue que $N / S$ não é finitamente gerado. Daí, existe $N^{\prime} / S$ subgrupo de $N / S$ com um número infinito enumerável de geradores, digamos $\overline{x_{1}}, \overline{x_{2}}, \ldots$ Considere a sequência de subgrupos finitamente gerados de $N / S$ (portanto de ordem finita).

$$
H_{1} / S \leq H_{2} / S \leq \ldots,
$$

onde $H_{j} / S=<\overline{x_{1}}, \ldots, \overline{x_{j}}>$. Como

- $H_{j}$ é um grupo sem $\mathbb{Z}_{2}$-torção (pois $H_{j} \leq G$ e $G$ não tem $Z_{2}$-torçâo por $1.5 .12(\mathrm{i}))$,

- $\left[H_{j}: S\right]<\infty$ (pois $N / S$ é localmente finito e $H_{j} / S$ é finitamente gerado) e,

- $S$ é um $D^{n-1}$-grupo,

segue de 1.5.19(i), que $H_{j}$ é um $D^{n-1}$-grupo para $j=1,2, \ldots$ Dai, por $1.5 .17, c d H_{j}=$ $n-1, H_{3}$ é de tipo $F P$ e $H^{k}\left(H_{j} ; \mathbb{Z}_{2} H_{j}\right)=0$ para $0 \leq k<n-1$, e $j=1,2, \ldots$

Além disso, a uniâo dos subgrupos $H_{1} \leq H_{2} \leq H_{3} \leq \ldots$ é $N^{\prime}$ (pois $\bigcup_{j=1}^{\infty} H_{j} / S=N^{\prime} / S$ e $S \subset H_{j}$ ), e $\left[H_{j+1}: H_{j}\right]=\left[H_{j+1} / S: H_{j} / S\right]<\infty$ (pois $\left.\left|H_{j} / S\right|<\infty, \forall j\right)$.

Logo por $\left[14\right.$, teor. 3.3], visto que $N^{\prime \prime}$ não é finitamente gerado (pois $N^{\prime \prime} / S$ nào é finitamente gerado) temos que

$$
c d N^{\prime}=(n-1)+1
$$

Portanto $c d N \geq c d N^{\prime}=n>c d S$. Dai por (d) temos que $[G: N]<\infty$, e então, como $G$ é finitamente gerado (condição (a)) segue que $N$ é finitamente gerado (cf. [25, 1.6.11]. Consequentemente $N / S$ é também finitamente gerado, o que é uma contradição.

Portanto a afirmação (1) é verdadeira, isto é, existe um subgrupo finitamente 
gerado $H / S$ de $N / S$ satisfazendo $c d H=n$.

Daj $c d N=n$ (pois $n=c d H \leq c d N \leq c d G \leq n$ ), e entào $[G: N]<\infty$ por (d), o que prova parte (i).

Vejamos (ii).

Como $S$ e $H / S$ sâo finitamente gerados segue que $H$ é também finitamente gerado.

Do fato que $S$ é um $D^{n-1}$-grupo segue que $S$ é de tipo $F P$ e $H^{j}\left(S ; \mathbb{Z}_{2} S\right)=0$ para $j \leq n-2$ (1.5.17).

Agora, $Q=H / S$ é infinito pois $n=c d H>c d S=n-1$. Logo, por 1.5.24(b), temos que $H / S$ tem mais que um end, isto é, $\epsilon(H / S) \geq 2$. Daí $H / S$ deve conter um elemento de ordem infinita (isto segue do teorema Stallings (teorema 1.4) e de [28, 1.1.3].

Seja $L / S$ o subgrupo cíclico infinito de $N / S$ gerado por este elemento.

Da sequência exata $S \gg L \rightarrow L / S \simeq \mathbb{Z}$. concluímos por I.5.19(i), que $L$ é um grupo de dualidade de dimensâo n. Dai $[G: L]<\infty$ (por (d)) e portanto temos $[N / S: L / S]=[N: L] \leq[G: L]<\infty, \operatorname{com} L / S \simeq \mathbb{Z}$, o que prova (ii).

Agora estamos em condiçòes de provar o resultado abaixo:

2.11 Teorema: $S \epsilon j a(G, S)$ um par grupo satisfazendo as condiçōes $(a),(b)$ 'e (d). Seja $C^{\prime}$ o módulo dualizante de $S$. Se $\left[\Lambda_{G}(S): S\right]<\infty$ entào $G$ é um $D^{n}$ grupo com módulo dualizante $C$ satisfazendo $\operatorname{Res} s_{S}^{G} C \simeq C^{\prime} \in$ portanto $H^{3}\left(G ; \mathcal{F}_{S} G\right) \simeq \mathbb{Z}_{2}$.

Demonstração: Nas hipóteses acima temos, pelo lema anterior, que $N_{G}(S) / S$ tem um subgrupo cíclico infinito $L / S$ de indice finito tal que $[G: L]<\infty$.

Consideremos a sequência exata curta

$$
0 \rightarrow S \rightarrow L \rightarrow L / S \simeq \mathbb{Z} \rightarrow 0
$$

Como $S$ é um $D^{n-1}$ grupo com módulo dualizante $C^{\prime}$ e $L / S$ é um $P D^{1}$-grupo segue que $L$ é um $D^{n}$-grupo com módulo dualizante $H^{n}\left(L ; \mathbb{Z}_{2} L\right) \simeq \mathbb{Z}_{2} \otimes C^{\prime} \simeq$ 
$C^{\prime}$, como $\mathbf{Z}_{2} L$-módulos (1.5.19(ii)).

Dai, como $G$ nào tem $\mathbf{Z}_{2}$-torçâa (pois $c d G \leq n$ ) e $[G: L]<\infty$ temos por 1.5.19 que $G$ é também um $D^{n}$-grupo com módulo dualizante $C=H^{n}\left(G ; \mathbb{Z}_{2} G\right)$ tal que $R e s_{L}^{G} C \simeq C^{\prime}$ (como $\mathbf{Z}_{2} L$-módulos). Assim, $S$ é um $D^{n-1}$-grupo com módulo dualizante $C^{\prime} \simeq R \varepsilon s_{S}^{G} C$, onde $C$ é o módulo dualizante de $G$.

Daí $H^{1}\left(G ; \mathcal{F}_{S} G\right) \simeq \mathbf{Z}_{2}$. (Para ver isto basta, na demonstraçào II.3.9(i) considerar $\mathcal{F}_{S} G=\operatorname{Ind} G P S$ no lugar de $\left.\mathbf{Z}_{2}(G / S)=\operatorname{Ind} G \mathbb{Z}_{2}\right)$.

Observação: Sabemos que se $G$ é um $P D^{n}$-grupo então $G$ é finitamente gerado de dimensâo cohomológica $n$ (ver 1.5 .15 e 1.5.18), e por um teorema de Strebel [33] todo subgrupo $K$ de índice infinito em $G$ satisfaz $c d K \leq n-1$. 0 teorema anterior nos dá uma recíproca parcial deste fato (quando $S$ é um $P D^{n-1}$-grupo).

Finalizando a secção, recordamos que em [21] §5, supondo que $G$ é um $P D^{n}$ grupo e $S$ é um $P D^{n-1}$-subgrupo, os autores provaram o seguinte fato:

2.12 Teorema: [21, teor. A] Sejam $G$ um $P D^{n}$-grupo e $S u m$ $P D^{n-1}$-subgrupo. Então $G$ se decompóe sobre um subgrupo comensurável com $S$ se $\epsilon$ somente se $\sin g_{G}(S)=0$.

Adaptando este resultado ao nosso invariante temos, analogamente:

2.13 Teorema: Sejam $G$ um $P D^{n}$-grupo e $S$ um $P D^{n-1}$-subgrupo. Então $G$ se decompôe sobre um subgrupo comensurável com $S$ se $\epsilon$ somente se $\hat{E}(G, S)=2$.

Demonstração: Consequência imediata do lema 2.3.

Assim. por exemplo, podemos concluir que $\mathbb{Z}^{k}$ se decompòe sobre um subgrupo comensurável com $\mathbb{Z}^{k-1}(k \geq 2)$ pois $E\left(\mathbb{Z}^{k}, \mathbb{Z}^{k-1}\right)=2$ (III.2.9). Similarmente, se $G$ e $S$ são como em II.3.12 entào $G$ se decompôe sobre um subgrupo comensurável com $S$.

Combinando 2.13 e 2.6 obtemos o seguinte resultado: 
2.14 Proposição: Scjam $G$ um grupo finitamente gcrado e $T$ C $S$ subgrupos de $G$ com $S \leq T \leq G,[G: T]<\infty$ e $[T: S]=\infty$. Se.

(i) $S$ e $T$ sáo finitamente gerados $e \tilde{E}(T, S)=1$ ou

(ii) $T$ é um $P D^{n}$-grupo, $S$ um $P D^{n-1}$-subgrupo, e $T$ não se decompõe sobre um subgrupo comensurável com $S$,

entâo $G$ nào se decompóe sobre um subgrupo comensurável com $S$.

Demonstração: (i) Pelo lema III.2.7, $\hat{E}(G, S) \leq \hat{E}(T, S)=1$. Dai o resultado segue do teorema 2.6. Agora (ii) segue de (i) pois, se $T$ nào se decompōe sobre um subgrupo comensurável com $S$ entào, pelo teorema anterior, $\tilde{E}(T, S)=1$. 


\section{BIBLIOGRAFIA}

[1] Andrade, M.G.C.; Tese de Doutorado, IMECC - Unicamp, Campinas (em preparação).

[2] Andrade, M.G.C.; Fanti, E.L.C.; A relative cohomological invariant for pairs of groups, (em preparação).

[3] Bieri, R.; Gruppen Mit Poincaré Dualität, Comm. Math. Helv. 47 (1972), 373 - 396.

[4] Bieri, R.; Homological dimension of discrete groups, Queen Mary College Math. Notes, Queen Mary College, London, (1976).

[5] Bieri, R.; Normal subgroups in duality groups and in groups of cohomological dimension 2, Journal of Pure and Applied Algebra 7 (1976), $35-51$.

[6] Bieri, R.; Eckmann, B.; Groups with homological duality generalizing Poincaré duality. Inventiones Math. 20 (1973), 103 - 124.

[7] Bieri, R.; Eckmann, B.; Relative homology and Poincaré duality, Journal of Pure and Applied Algebra 13 (1978), 277 - 319.

[8] Brown, K.S.; Cohomology of groups, G.T.M. 87 Springer-Verlag, New York, (1982).

[9] Cohen, D.F.; Groups of cohomological dimension one, Lectures Notes in Math. 245 Springer-Verlag, Berlin (1972).

[10] Dicks, W.; Dunwood, M.; Groups acting on graphs, Cambridge Unver- 
sity Press, (1988).

[11] Eckmann, B.; Müller, H.; Poincaré duality groups of dimension two, Comm. Math. Helvetici 55, (1980), 510 - 520.

[12] Eckmann, B.; Linell, P.A.; Poincaré duality groups of dimension two II, Comm. Math. Helv. 58, (1983), 111 - 114.

[13] Freudenthal, H; Über die Enden Topologischer Raüme und Gruppen, Math. Zeit. 33 (1931), 692 - 713.

[14] Gildenhuys, D.; Strebel, R.; On the cohomological dimension of soluble groups, Canad. Math. Bull. Vol. 24(4) (1981), 385 - 392.

[15] Hilton, P.J.; Wylie, S.; Homology Theory - An Introduction to Algebraic Topology, Cambridge Univ. Press (1960).

[16] Hopf, H.; Enden offener Raüme und unendlicher Gruppen, Comm. Math. Helv. 16 (1943), $81-100$.

[17] Houghton, C.H.; Ends of locally compact groups and their coset spaces, J. Aust. Math. Soc. 17 (1974) $274-284$.

[18] Hu, S.T.; Introduction to homological algebra, Holden-Day series in Mathematics, Holden-Day inc. Sâo Francisco, (1968).

[19] Johnson F.E.A.: Wall, C.T.C.; On groups satisfying Poincaré duality, Annals of Math., 96 (1972), 592 - 598.

[20] Kropholler, P.H.; An analogue of the torus decomposition theorem for certain Poincaré duality groups, Proc. London Math. Soc. 3 (1990), $503-529$.

[21] Kropholler, P.H,; Roller, M.A.; Splittings of Poincaré duality groups, Math. Z. 97 (19SS), 421 - 438.

[22] Kropholler, P.H.; Roller, M.A.; Relative ends and duality groups, 
Journal of Pure and Appl. Algebra, 61 (1989), 197 - 210.

[23] Raymond, F.; Scott, L.L.; Failure of Nielsen's theorem in higher dimensions, Arch. Math., 29(6) (1977), 643 - 654.

[24] Ribes, L.; On a cohomology theory for pairs of groups, Proc. of the A.M.S. 21 (1969) 230 - 234.

[25] Robinson, D.J.S.; A course in the theory of groups, G.T.M. 80, Springer-Berlin, (19S2).

[26] Scott, G.P.; Ends of pairs of groups, J. Pure Appl. Algebra 11 (1977), $179-198$.

[27] Scott, G.P.; Wall, C.T.C.; Topological methods in group theory, London Math. Soc., Lect. Notes Series 36, Homological Group Theory, (1979) 137 - 203.

[28] Serre, J.P.; Trєєs, Springer-Verlag, Berlin Heidelberg New York (1980).

[29] Siebenmann, L.C.; The obstruction to finding a boundary for an open manifold of dimension greater than five, $\mathrm{PhD}$ Thesis, Princeton Univ. Press (1965).

[30] Specker, E.; Endenverbände van Raümє und Gruppen, Math. Ann. 122 (1950), $167-174$.

[31] Stallings, J.R.; On torsion - free groups with infinitely many ends, Ann. Math. S8 (1968), 321 - 334.

[32] Stallings, J.R.; Groups theory and 3-dimensional manifolds, Yale Univ. Press, (1971).

[33] Strebel, R.; A remark on subgroups of infinte index in Poincaré duality groups, Comment. Math. Helvetici 52 (1977), 317 - 324.

[34] Thomas, C.B.; Splittings theorems for certain $P D^{3}$-groups, Math. Z. 
186, (1984), 201 209.

[35] Whitehead, G.W.; Homolopy Theory, The M.J.T. Press, Massachusetts (1966).

[36] Whitehead, G.W.; Elements of homotopy theory, G. T. M. 61, SpringerVerlag, New York, (1978). 